University of Tennessee Health Science Center

UTHSC Digital Commons

\title{
Autoimmune Consequences of Histone Deimination during Neutrophil Activation
}

\author{
Nishant Dwivedi \\ University of Tennessee Health Science Center
}

Follow this and additional works at: https://dc.uthsc.edu/dissertations

Part of the Immune System Diseases Commons, and the Medical Immunology Commons

\section{Recommended Citation}

Dwivedi, Nishant , "Autoimmune Consequences of Histone Deimination during Neutrophil Activation" (2011). Theses and Dissertations (ETD). Paper 69. http://dx.doi.org/10.21007/etd.cghs.2011.0077. 


\title{
Autoimmune Consequences of Histone Deimination during Neutrophil Activation
}

\begin{abstract}
Tolerance blocks the expression of autoantibodies, whereas autoimmunity promotes it. How tolerance breaks and autoantibody production begins, thus, are crucial questions for the understanding and treatment of autoimmune diseases. Evidence implicates cell death and autoantigen modifications in the initiation of autoimmune reactions. One form of neutrophil cell death deserves attention because it occurs as a consequence of neutrophil activation, requires the post-translational modification of histones and results in the extracellular release of chromatin. The extracellular chromatin incorporates histones in which arginines have been converted to citrullines by peptidylarginine deiminase IV (PAD4) creating structures that capture or "trap" bacterial pathogens. Neutrophil extracellular traps (NETs), as these structures are known, generate an extracellular complex of deiminated histones and bacterial cell adjuvants. The complex of bacterial antigens and deiminated chromatin may be internalized by host phagocytes during inflammatory conditions, as arise during bacterial infections or chronic autoinflammatory disorders. The uptake and processing of deiminated chromatin together with bacterial adjuvants by phagocytes may induce the presentation of modified histone epitopes and co-stimulation, thus yielding a powerful stimulus to break tolerance. To test the hypothesis that NETs can lead to autoimmunity, we measured autoantibodies to deiminated histones in human autoimmune disorders. We detected autoantibodies to deiminated histones in Felty's syndrome (FS) patients, whereas autoantibodies from Systemic Lupus Erythematosus (SLE) and Rheumatoid Arthritis (RA) patients did not distinguish deiminated from non-deiminated histones. FS autoantibodies colocalized with deiminated histone H3 in LPS-treated neutrophils suggesting activated neutrophils as the source of autoantigens. In addition, we identified and characterized deimination of linker histone $\mathrm{H} 1$ and found rare autoantibodies to deiminated $\mathrm{H} 1$ in SLE and Sjogren's syndrome patients. We also studied sera of autoimmune lupus prone mice to determine if they would recognize deiminated histones and found that deimination represses binding of murine lupus autoantibodies to histones. The inability of immunoglobin from sera of lupus mice to recognize deiminated histones suggests the presence of effective tolerance to NET components released during innate neutrophil response to infections. Our finding of antibodies to deiminated histones in Felty's syndrome supports the idea that tolerance to deiminated histones is compromised only in exceptional circumstances. Understanding the tolerance mechanism to deiminated histones and how they are compromised in patients could be useful to design strategies for the prevention and treatment of many autoimmune disorders.
\end{abstract}

Document Type

Dissertation

Degree Name

Doctor of Philosophy (PhD)

Program

Biomedical Sciences

Research Advisor

Marko Z. Radic, Ph. D.

Keywords

Autoimmunity; Deimination; Felty's Syndrome; Histone; PAD4; Tolerance 


\section{Subject Categories}

Diseases | Immune System Diseases | Medical Immunology | Medical Sciences | Medicine and Health Sciences 


\title{
AUTOIMMUNE CONSEQUENCES OF HISTONE DEIMINATION DURING NEUTROPHIL ACTIVATION
}

\author{
A Dissertation \\ Presented for \\ The Graduate Studies Council \\ The University of Tennessee \\ Health Science Center
}

\begin{abstract}
In Partial Fulfillment
Of the Requirements for the Degree

Doctor of Philosophy

From The University of Tennessee
\end{abstract}

By

Nishant Dwivedi

December 2011 
Chapter 2 (C) 2011 by the American College of Rheumatology. All other materials $(\mathcal{C} 2011$ by Nishant Dwivedi. All rights reserved. 


\section{DEDICATION}

This dissertation is dedicated to my parents,

Prof. Ram Prakash Dwivedi and Mrs. Braj Prabha Dwivedi

for their endless love, support and encouragement

in my pursuit of knowledge and excellence. 


\section{ACKNOWLEDGEMENTS}

I would like to acknowledge all those who have supported me during my research. I owe my deepest gratitude to my mentor, Dr. Marko Radic for accepting me in his lab as a student. His support and guidance throughout my graduate years have been exemplary. He steered my project while giving me enough opportunities and freedom to develop my own ideas. He helped me develop scientific and analytical thinking which will go a long way to shape the rest of my life.

I am also grateful to my committee members, Dr. Elizabeth Fitzpatrick, Dr. Fabio Re, Dr. John Stuart and Dr. Terrence Geiger. Their constructive criticism and meaningful suggestions helped me a lot in various stages of my project. I am also thankful to my collaborators, without them this study would not have been possible. I extend my thanks to Dr. Paul Thompson at the Scripps Institute, Florida who provided me with the recombinant PAD4 enzyme. I sincerely appreciate the help of Dr. Elena Csernok (Germany), Dr. Mary Crow, Dr. Kyriakos Kirou (Hospital for Special Surgery, NY), Dr. Linda Myers, Dr. Debendra Pattanaik (UTHSC) and Dr. Ted Mikuls (University of Nebraska) in providing me with patient samples for this study. The support I received from Dr. Ole Peter Rekvig (Norway), Dr. Laurence Morel (University of Florida) and Dr. Chandra Mohan (UT Southwestern) for my animal studies was invaluable. I would also like to thank Dr. Dom Desiderio and Mr. Haibao Wan of UTHSC Mass Spectrometry facility for their help in my experiments. I am also thankful to Dr. Syllviane Muller (France) for the generous gift of synthetic peptides that helped me to complete crucial experiments.

Next, I would like to thank my present and previous colleagues in Radic lab, Dr. Indira Neeli and Dr. Salar Khan for their help and guidance in various experiments. The time I spent with them in the lab working on my project and learning new things will remain memorable. I would also thank Tim Higgins for helping me with the illustrations.

I am forever indebted to my parents Prof. Ram Prakash Dwivedi, Mrs. Braj Prabha Dwivedi and other family members for their endless patience and encouragement in my pursuit of science. My special thanks to my brother Mr. Neelam Dwivedi who has been a continuous source of inspiration and motivation to me and who has always encouraged me to strive for academic excellence. Without his support I would have perhaps not entered graduate school to pursue a Ph. D. I am also thankful to my wife, Jagriti Upadhyay, for the love, support and personal sacrifices she made for successful completion of my research project. I also thank all my friends for being the surrogate family during all my graduate years and for their continuous moral support. I also take the opportunity to acknowledge with gratitude all my past and present teachers who played an important role in shaping up my personality and career. Finally, I would like to acknowledge Lupus Research Institute and Dana Foundation Program in Human Immunology for funding my research project. 


\begin{abstract}
Tolerance blocks the expression of autoantibodies, whereas autoimmunity promotes it. How tolerance breaks and autoantibody production begins, thus, are crucial questions for the understanding and treatment of autoimmune diseases. Evidence implicates cell death and autoantigen modifications in the initiation of autoimmune reactions. One form of neutrophil cell death deserves attention because it occurs as a consequence of neutrophil activation, requires the post-translational modification of histones and results in the extracellular release of chromatin. The extracellular chromatin incorporates histones in which arginines have been converted to citrullines by peptidylarginine deiminase IV (PAD4) creating structures that capture or "trap" bacterial pathogens. Neutrophil extracellular traps (NETs), as these structures are known, generate an extracellular complex of deiminated histones and bacterial cell adjuvants. The complex of bacterial antigens and deiminated chromatin may be internalized by host phagocytes during inflammatory conditions, as arise during bacterial infections or chronic autoinflammatory disorders. The uptake and processing of deiminated chromatin together with bacterial adjuvants by phagocytes may induce the presentation of modified histone epitopes and co-stimulation, thus yielding a powerful stimulus to break tolerance.

To test the hypothesis that NETs can lead to autoimmunity, we measured autoantibodies to deiminated histones in human autoimmune disorders. We detected autoantibodies to deiminated histones in Felty's syndrome (FS) patients, whereas autoantibodies from Systemic Lupus Erythematosus (SLE) and Rheumatoid Arthritis (RA) patients did not distinguish deiminated from non-deiminated histones. FS autoantibodies colocalized with deiminated histone H3 in LPS-treated neutrophils suggesting activated neutrophils as the source of autoantigens. In addition, we identified and characterized deimination of linker histone $\mathrm{H} 1$ and found rare autoantibodies to deiminated H1 in SLE and Sjogren's syndrome patients. We also studied sera of autoimmune lupus prone mice to determine if they would recognize deiminated histones and found that deimination represses binding of murine lupus autoantibodies to histones. The inability of immunoglobin from sera of lupus mice to recognize deiminated histones suggests the presence of effective tolerance to NET components released during innate neutrophil response to infections. Our finding of antibodies to deiminated histones in Felty's syndrome supports the idea that tolerance to deiminated histones is compromised only in exceptional circumstances. Understanding the tolerance mechanism to deiminated histones and how they are compromised in patients could be useful to design strategies for the prevention and treatment of many autoimmune disorders.
\end{abstract}




\section{TABLE OF CONTENTS}

CHAPTER 1. INTRODUCTION ....................................................................................1

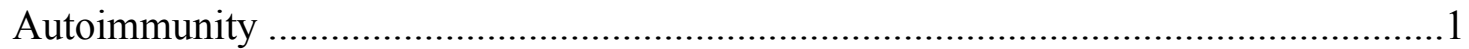

Types of Autoimmune Disorders ...........................................................................2

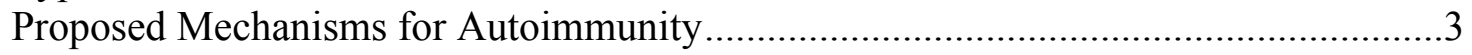

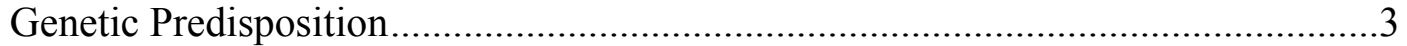

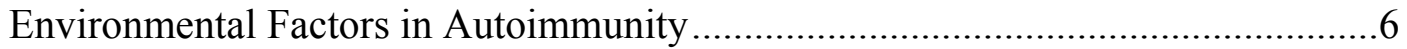

Post Translational Modification of Proteins: Generation of "Neo-epitopes"............6

Cell Death, Exposure of Hidden Antigens and Defective Clearance....................... 7

Autoimmunity: Interplay of Innate and Adaptive Immunity ....................................

Immune Response to Infectious Agents and Lessons for Autoimmunity ...............10

Successful Adaptive Immune Response Requires Contribution from Innate

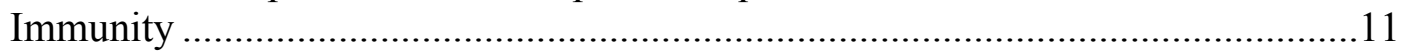

Components of Innate Immunity Involved in Autoimmunity and Tolerance .........11

Infections Generate an Environment Suitable for Autoimmunity .............................12

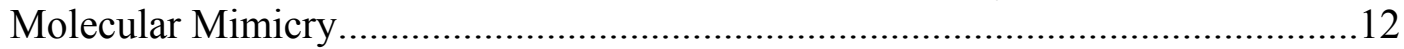

Bystander Activation and Epitope Spreading ..................................................... 13

Cryptic Antigens and Epitopes........................................................................ 14

Neutrophils: First Line of Defense against Infections.........................................15

Neutrophil Engagement of Infectious Agents: Mechanisms and Consequenses ....16

Neutrophils as Antigen Presenting Cells .........................................................17

Neutrophil Extracellular Traps: Unique Mechanism to Counter Infections ...........17

Deimination: a Post Translational Modification during Neutrophil Activation............19

Physiological Effects of Protein Deimination ..................................................19

Deiminated Proteins and Autoimmunity .........................................................19

Neutrophil Activation: Confluence of Factors for Initiation of Autoimmunity

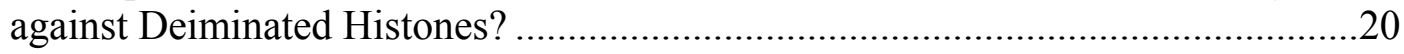

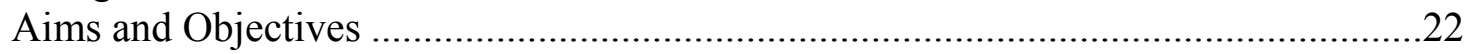

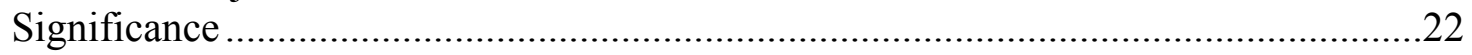

\section{CHAPTER 2. FELTY'S SYNDROME AUTOANTIBODIES BIND TO DEIMINATED HISTONES AND NEUTROPHIL EXTRACELLULAR TRAPS...25}

Introduction 
Preferential Antibody Binding to Deiminated Histones in ELISA .........................33

Autoantibodies Distinguish between Different Deiminated Histones ....................33

Autoantibodies from ANCA-associated Vasculitides Prefer Non-deiminated

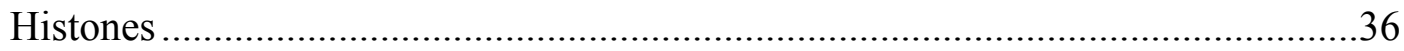

LPS Stimulation of Neutrophils Generates Preferred Histone Autoepitopes..........39

Deimination Is Responsible for Preferential Histone Binding ..............................39

Circulating Neutrophils from RA Patients Show Spontaneous Activation.............42

Discussion

\section{CHAPTER 3. AUTOANTIBODY RECOGNITION OF CITRULLINE IN THE GLOBULAR DOMAIN OF LINKER HISTONE H1 .............................................46}

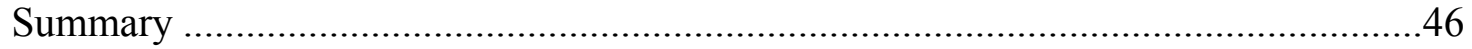

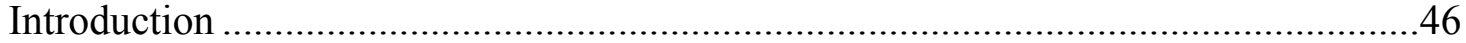

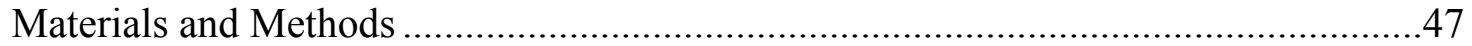

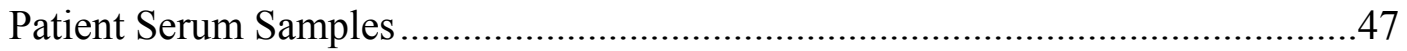

In-vitro Deimination of Recombinant H1.2 ….................................................48

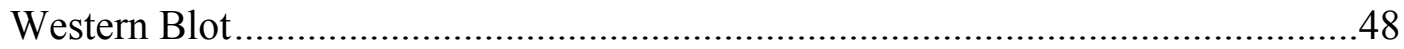

Preparation of Protease Digested Peptides .....................................................48

Liquid Chromatography-Mass Spectrometry and Database Search.......................49

RNA Extraction and Microarray Analysis .........................................................49

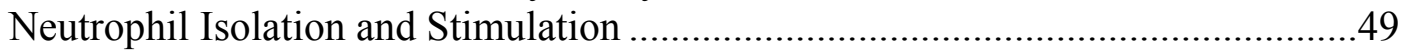

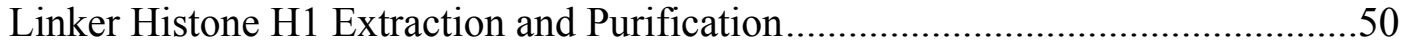

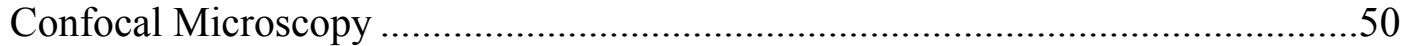

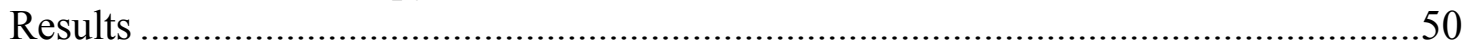

Identification of Autoantibodies to Deiminated H1 2 .......................................50

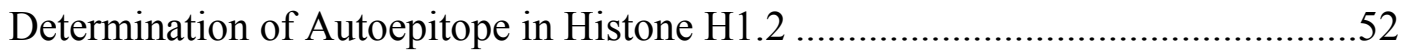

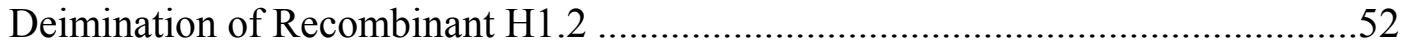

Autoimmune IgG Preferentially Bind H1 Linker Histones in Activated

Neutrophils and Neutrophil Extracellular Chromatin ......................................56

Neutrophils Express Multiple H1 mRNAs and Proteins ....................................56

MS/MS Analysis of H1 Histones from Activated Neutrophils .............................59

Summary of Histone H1 Expression in Neutrophils ..........................................59

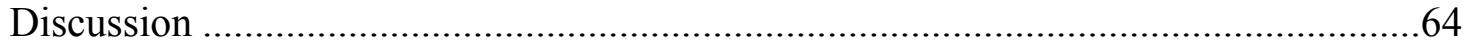

\section{CHAPTER 4. DEIMINATION REDUCES REACTIVITY OF HISTONES WITH MURINE LUPUS AUTOANTIBODIES .......................................................66}

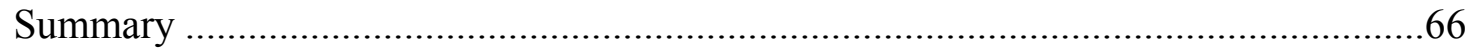

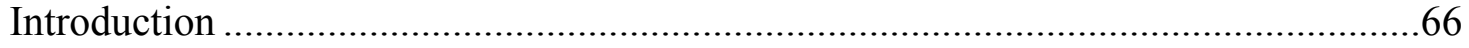

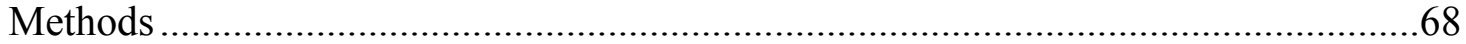

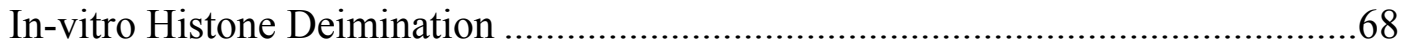

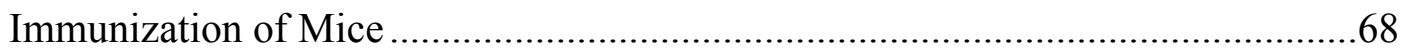

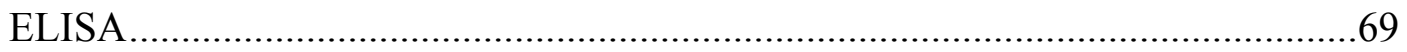



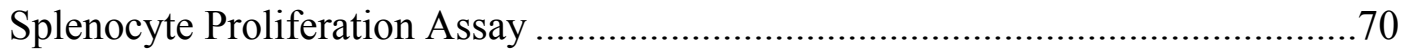

Ex-vivo Tissue Lysate Preparation...................................................................... 70

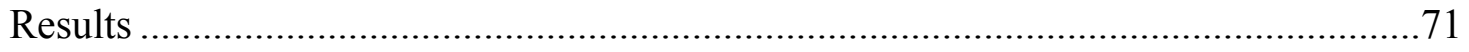


In-vitro Histone Deimination

Spontaneously Arising Anti-histone Autoantibodies Bind Non-deiminated

Histones

(NZBxNZW) F 1 Splenocytes Respond to Deiminated Histones or Non-

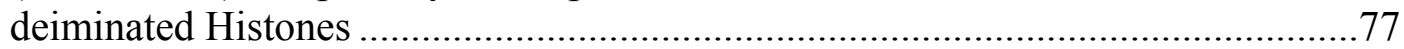

Spleens of Autoimmune Mice Have Increased Levels of Deiminated Histones ....80

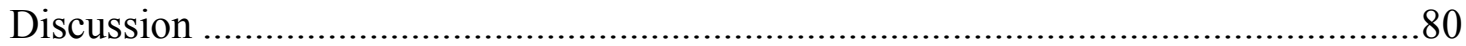

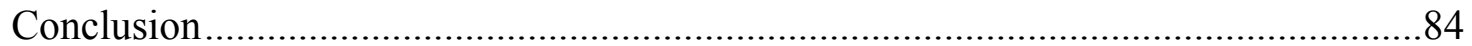

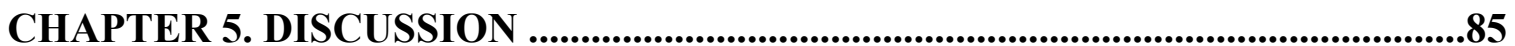

Post-Translational Modification of Autoantigens .............................................. 85

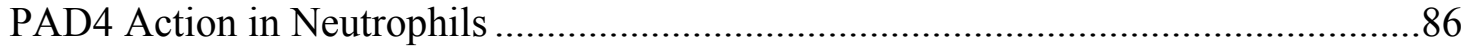

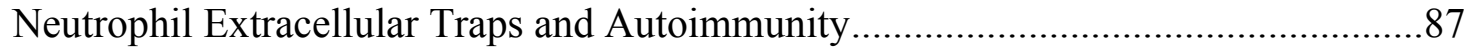

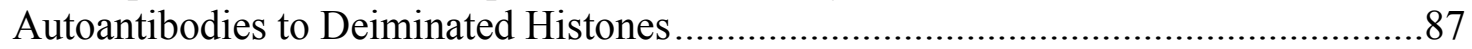

Lupus Mice Do Not Make Antibodies to Deiminated Histones .................................89

Splenocytes of Autoimmune Mice Respond to Both Deiminated and Non-

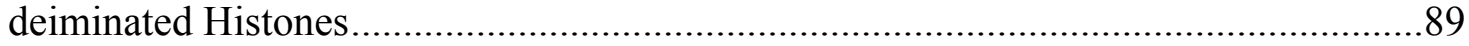

Presence of Deiminated Histones in Lymphoid Organs of Autoimmune Mice............90

$\mathrm{B}$ and $\mathrm{T}$ Cell Responses in BALB/c Mice Immunized with Deiminated Histones.......91

Deiminated Linker Histones in Neutrophils.............................................................91

Autoantibodies to Deiminated Linker Histones ..................................................92

Inferences from Human and Mice Antibody Response to Deiminated Histones..........93

Model of Autoimmune Pathogenesis in Felty's Syndrome.......................................94

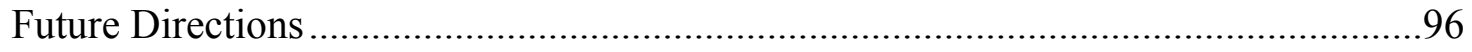

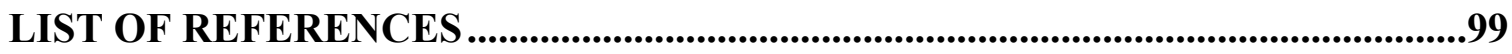

VITA 


\section{LIST OF TABLES}

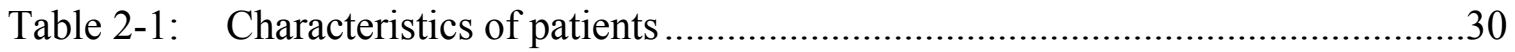

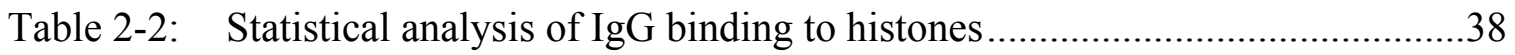

Table 3-1: Arginine containing peptides detected in $\mathrm{H} 1$ extracted from activated

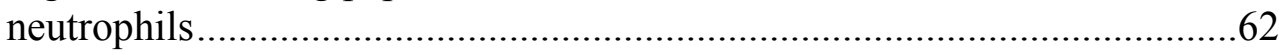

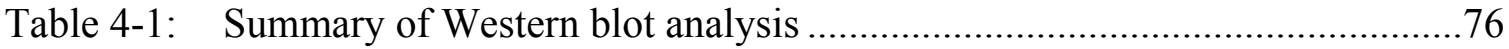




\section{LIST OF FIGURES}

Figure 2-1: Binding of autoimmune sera to activated neutrophils................................. 31

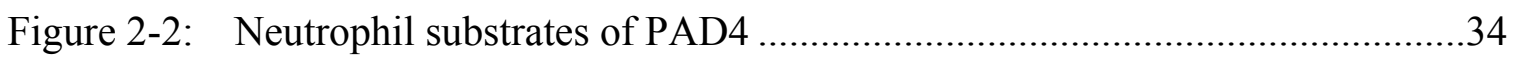

Figure 2-3: Serum IgG binding to deiminated or non-deiminated histones ...................35

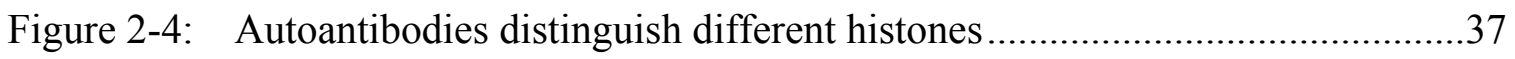

Figure 2-5: FS2 IgG prefer activated neutrophils, deiminated recombinant histone

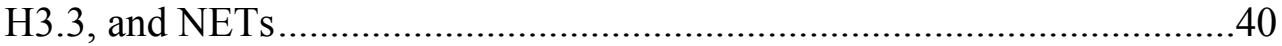

Figure 2-6: Deiminated histone H3 in circulating neutrophils and neutrophil stimulation by autoimmune sera ......................................................43

Figure 3-1: Preferential binding of autoimmune sera to deiminated H1.2 …...............51

Figure 3-2: Peptide inhibition of SLE6 binding to deiminated H1.2 ...........................53

Figure 3-3: $\quad$ MS/MS analysis of in vitro deiminated recombinant $\mathrm{H} 1.2 \ldots \ldots \ldots \ldots \ldots \ldots \ldots \ldots . . .54$

Figure 3-4: Neutrophil activation increases SLE6 reactivity ......................................57

Figure 3-5: Analysis of linker histone transcripts and proteins from neutrophils .........58

Figure 3-6: MS/MS analysis of H1 peptides from activated neutrophils .....................60

Figure 3-7: Summary of mass spectrometry with linker histones from activated neutrophils....

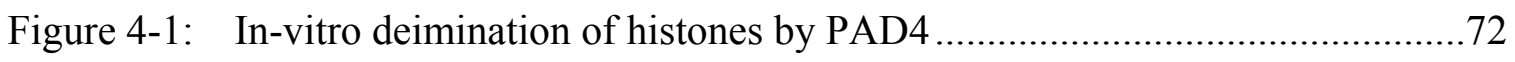

Figure 4-2: Binding of IgG from autoimmune prone and control mice to nondeiminated histones and deiminated histones

Figure 4-3: Western blot of IgG to deiminated histones and non-deiminated histones..74

Figure 4-4: Proliferation of splenocytes from $\left(\mathrm{NZBxNZW) \textrm {F } _ { 1 }}\right.$ mice...........................78

Figure 4-5: $\quad$ Proliferation of splenocytes from immunized BALB/c mice ....................79

Figure 4-6: B cell response after $3^{\text {rd }}$ immunization using Imiquimod, a TLR-7 agonist as adjuvant ................................................................... 81

Figure 4-7: Western blot detection of deiminated histones $\mathrm{H} 3$ in mouse tissues ...........82

Figure 5-1: A model of autoimmune pathogenesis in Felty's syndrome ......................95 


\section{LIST OF ABBREVIATIONS}

AAV

AChR

AIRE

ANCA

APC

APECED

APRIL

AS

BAFF

BCR

BLyS

C4

CFA

CGD

CMV

CSS

CTLA4

CVB

$\mathrm{dH}$

dsDNA

EAE

EBV

ERK1/2

FDC

FS

GBS

HLA

HMGB1

hsp70

IFA

Ig

IL

IRAK 1

IRBP

IRF

LPS

MER-TK

MGF-E8

$\mathrm{MHC}$

MMP

MPA

MS
(ANCA)-associated Vasculitides

Acetylcholine Receptor

Autoimmune Regulator

Anti-Neutrophilic Cytoplasmic Antibodies

Antigen Presenting Cells

Autoimmune Polyglandular Endocrinopathy with Candidiasis and

Ectodermal Dysplasia

A Proliferation Inducing Ligand

Ankylosing Spondyloarthopathy

B Cell Activating Factor

B Cell Receptor

B-lymphocyte stimulator

Complement 4

Complete Freund's Adjuvant

Chronic Granulomatous Disease

Cytomegalovirus

Churg-Strauss Syndrome

Cytotoxic T-Lymphocyte Antigen 4

Coxsackievirus B

Deiminated Histone

Double Stranded Deoxyribo Nucleic Acid

Experimental Autoimmune Encephalomyelitis

Epstein-Barr Virus

Extracellular Signal-Regulated Kinases 1 and 2

Follicular Dendritic Cells

Felty's Syndrome

Guillian-Barre Syndrome

Human Leukocyte Antigen

High Mobility Group Protein B1

Heat Shock Protein 70

Incomplete Freund's Adjuvant

Immunoglobulin

Interleukin

Interleukin-1 Receptor-associated kinase 1

Interphotoreceptor Retinoid Binding Protein

Interferon Regulatory Factor

Lipopolysaccharide

c-Mer Proto-oncogene Receptor Tyrosine Kinase

Milk Fat Globule E8

Major Histocompability Complex

Matrix Metalloprotinases

Microscopic Polyangiitis

Multiple Sclerosis 


$\begin{array}{ll}\text { NADPH } & \text { Nicotinamide Adenine Dinucleotide Phosphate } \\ \text { nH } & \text { Non-deiminated Histone } \\ \text { NOD2 } & \text { Nucleotide Binding and Oligomerization Domain 2 } \\ \text { OVA } & \text { Ovalbumin } \\ \text { PAD } & \text { Peptidylarginine deiminase } \\ \text { PAF } & \text { Platelet Activating Factor } \\ \text { PAMP } & \text { Pathogen Associated Molecular Patterns } \\ \text { PCH } & \text { Paroxysmal Cold Hemoglobinuria } \\ \text { PGE2 } & \text { Prostaglandin E2 } \\ \text { PLP } & \text { Proteolipid Protein } \\ \text { PMN } & \text { Polymorphonuclear (cells) } \\ \text { PR3 } & \text { Proteinase 3 } \\ \text { PRR } & \text { Pattern Recognition Receptors } \\ \text { PS } & \text { Phosphotidylserine } \\ \text { PTM } & \text { Post Translational Modification } \\ \text { RA } & \text { Rheumatoid Arthritis } \\ \text { RBC } & \text { Red Blood Cells } \\ \text { SKIV2L } & \text { Super Killer Viralicidic activity 2-like Gene } \\ \text { SLE } & \text { Systemic Lupus Erythematosus } \\ \text { SnRNP } & \text { Small Nuclear Ribonucleoprotein } \\ \text { SOCS } & \text { Suppressor of Cytokine Synthesis } \\ \text { SS } & \text { Sjogren's Syndrome } \\ \text { STAT4 } & \text { Signal Transducer and Activator of Transcription 4 } \\ \text { T1D } & \text { Type 1 Diabetes } \\ \text { TGF- } \beta & \text { Transforming Growth Factor-Beta } \\ \text { TLR } & \text { Toll-like Receptor } \\ \text { TMEV } & \text { Theiler's Murine Encephalomyelitis Virus } \\ \text { TNF- } \alpha & \text { Tumor Necrosis Factor Alpha } \\ \text { TSH } & \text { Thyroid Stimulating Harmone } \\ \text { WG } & \text { Wegener's Granulomatosis } \\ & \end{array}$




\section{CHAPTER 1. INTRODUCTION}

\section{Autoimmunity}

In 1901 Erhlich coined the term "Horror Autotoxicus" to explain "the unwillingness of the organism to endanger itself by formation of toxic autoantibodies". He found that immunizing goats with their own red blood cells (RBCs) did not lead to any antibody formation (1). Erhlich postulated that autoimmune diseases cannot occur and lack of immune response against self antigens came to be known as 'tolerance'. However, the first autoimmune disorder was reported in 1904, when Donath and Landsteiner demonstrated that toxic autoantibodies destroy self RBCs in Paroxysmal Cold Hemoglobinuria (PCH) (2). For the next 50 years, the study of autoimmune diseases progressed slowly until Rose and Roitt nearly simultaneously published separate reports on autoimmune thyroiditis. Rose and Witebsky published evidence for the experimental induction of autoimmune thyroiditis in rabbits $(3,4)$. About the same time, Roitt published a report on thyroid-reacting autoantibodies in Hashimoto's patients (5). Identification of Hashimoto's thyroiditis as an autoimmune condition led to acceptance to the concept of autoimmunity and opened up the search for others.

The acceptance of autoimmunity forced scientists to look at the immune system in a new light. Burnet explained that immune response (antibody production) results after engagement and expansion of clones from a cell with a single specificity (Clonal Selection) (6) and tried to explain tolerance as a result of "deletion" of self reactive clones from the B cell repertoire. He postulated that autoimmunity results from the escape of "forbidden" self reacting clones. Cloning of B cells of healthy individuals revealed that about $76 \%$ of early immature B cells express self reactive antibody receptors (7). Self reactivity of B cell receptors (BCR) progressively decreased as B cell development progressed. The first check point against self reactivity was observed during the transition of immature B cells to mature B cells as the frequency of self reactive antibodies produced falls to about $40 \%$ in B cells newly emigrated from the bone marrow (7). Another checkpoint for getting rid of self reacting specificities was found during the maturation of newly emigrant B cells to peripheral naïve B cells (7). In patients with SLE, about $25-50 \%$ of naïve B cells express self-recognizing BCR in contrast to about $5-20 \%$ of B cells from healthy individuals (8). Though these observations tried to explain autoimmunity on the basis of escape of self reacting B cells, more questions than answers ensued. What role do self-reactive naïve B cells play in healthy individuals? In what way are these cells different in healthy individuals and autoimmune patients? How are the cells bearing self-reactive receptors kept in check in healthy individuals? It follows that various mechanisms contribute to keep the selfreactive lymphocytes in a state of non-responsiveness called "anergy". Engagement of lymphocyte receptors by self-antigens without receiving a second signal (from an activated helper T cells or CD80/86 expressed on APC) promotes desensitization of receptor for subsequent re-exposure to antigen. Chronic engagement of BCR with selfantigen results in low calcium influx and activation of inhibitory signaling that prevents activation of B cells $(9,10)$. Survival and proliferation of B cells on receptor engagement 
is dependent on B cell activating factor (BAFF; also known as BLyS) and self-reacting B cells have a higher threshold for BAFF signaling and so they are outcompeted by naïve B cells for survival in lymphoid follicles (11). Many more mechanisms for maintaining peripheral tolerance have been found (12-14) and force us to think about other mechanisms by which autoimmune disorders arise. What conditions favor the engagement and activation of self-reactive B cells leading to the initiation of autoimmune diseases? Presently, we have some knowledge about factors that contribute to autoimmunity, yet understanding how autoimmune responses are initiated and perpetuated is far from clear.

Owing to the self perpetuating nature of autoimmunity in which the targeted antigen cannot be cleared from the body, autoimmune responses are extremely destructive. The autoimmune disorders cause extensive morbidity and can sometimes become life threatening. The chronic morbidity of autoimmune diseases hampers the normal lifestyle and productivity of millions of affected people. In the past hundred years, ever since the discovery of the first autoimmune condition, many more disease states have been identified that have an autoimmune component.

\section{Types of Autoimmune Disorders}

Presently, there are around 80 autoimmune diseases known in humans (15). The prevalence of autoimmune disorders is estimated to be about $5 \%$ in the world population $(16,17)$. The current classification of autoimmune disorders is based on the organs involved, the type of lesion, the morbidity and also the type of autoimmune process involved. Broadly, autoimmune disorders are grouped into organ-specific or systemic autoimmune disorders. In organ-specific disorders, the autoimmune immune response is directed against an antigen that is localized to one particular organ. The classical example of such an organ-specific autoimmune disorder is Hashimoto's thyroiditis in which the thyroid gland gradually becomes dysfunctional. Autoantibodies against thyroid-specific antigens, thyroid peroxidase, thyroglobulin and thyroid stimulating hormone (TSH) receptor, are found in patients with Hashimoto's thyroiditis. Autoantibodies mediate the destruction of the thyroid resulting in hypothyroidism. Other organ-specific autoimmune disorders include type 1 Diabetes Mellitus (T1D), Graves' disease and Autoimmune Hemolytic Anemia.

In systemic autoimmunity, the autoimmune response is directed against a target antigen that is widespread in its distribution. Systemic lupus erythematosus (SLE) is a prototype of systemic autoimmunity and is characterized by autoantibodies against double stranded deoxyribonucleic acid (dsDNA), histones and a variety of other nuclear antigens that are found in every tissue of the body. The main organ systems involved in SLE are the kidneys, heart, joints, skin, lung, blood vessels and the nervous system. Other systemic autoimmune disorders are Rheumatoid Arthritis (RA), Sjogren's syndrome (SS) and Dermatomyositis. The development of organ-specific or systemic autoimmunity depends on the autoantigens targeted by the immune system and their distribution within the body. In some autoimmune conditions, the cells bearing the 
targeted antigen are distributed to all parts of the body by the circulation leading to widespread disease manifestations. Classic examples of such autoimmune conditions are the small vessel vasculitides that involve neutrophil antigens and are characterized by the presence of anti-neutrophilic cytoplasmic antibodies (ANCA). Three subtypes of small vessel vasculitides are Wegener's granulomatosis (WG), Churg-Strauss syndrome (CSS) and microscopic polyangiitis (MPA). All three of them are characterized by severe inflammation and granuloma formation in the kidney, lungs, upper respiratory tract, gastrointestinal tract and skin.

In order to provide effective clinical treatment to millions of patients with autoimmune diseases, it is necessary to understand how and when some self antigens become targets of the immune response. A better insight into what drives a particular autoimmune process will help in preventing autoimmune conditions and guide the development of new treatments.

\section{Proposed Mechanisms for Autoimmunity}

Autoimmunity has a complex etiology. Genetics and environmental factors play roles in the engagement, activation and proliferation of self-antigen specific lymphocytes resulting in autoimmunity. Several examples of genetic defects predisposing to autoimmunity have been described. For example, the src-family tyrosine kinase lyn, which is associated with membrane immunoglobulin (Ig), is essential for B cell signaling, and defects in lyn signaling may result in B cell activation by self-antigens and autoimmunity (18). T cells express an inhibitory receptor, cytotoxic T-lymphocyte antigen (CTLA4) that competes with CD28 for binding to CD80/86 molecules and transmits inhibitory signals (19). Polymorphisms in CTLA4 gene were found to be associated with Grave's disease as well as T1D (20). In one mouse model of T1D a variation of CTLA4 mRNA splicing resulting in a molecule with reduced ability to interact with CD80/CD86 ligands were found (20). With development of new biological techniques for studying autoimmunity, the following mechanisms have been proposed to influence initiation of autoimmunity.

\section{Genetic Predisposition}

Genetic predisposition plays an important role in the development of autoimmune disorders. Multiple polymorphisms in major histocompability complex (MHC) genes, the most extensively studied region of human genome, have been found associated with autoimmune disorders. In human genes encoding $\mathrm{MHC}$ molecules are located on the short arm of chromosome 6 (21). MHC gene loci are segregated into 3 different classes, with Class 1 including genes for human leukocyte antigens (HLA) A, B and C. Class II MHC consists of genes for heterodimeric HLA-DR, DP and DQ molecules. Many other gene products including complement components (eg $\mathrm{C} 4$ ), tumor necrosis factor alpha (TNF- $\alpha$ ) and heat shock protein 70 (hsp70) are grouped together in MHC Class III (22). With genes involved in signaling, cell death and antigen processing and presentation 
closely clustered in one small region of the genome, polymorphisms in MHC molecules influence immunoreactivity against many self antigens. As early as the seventh decade of the $20^{\text {th }}$ century, association of HLA-B27 with ankylosing spondyloarthopathy (AS) was established $(23,24)$. The association of HLA-B27 or its subtypes with AS holds true worldwide with higher prevalence of AS in populations with higher frequency of HLAB27 (25). Even after this close association between AS and HLA-B27, a genetic study found that only $16 \%$ of identical twins with HLA-B27 positive genotype developed AS (26). More recently, it was found that susceptibility to AS is not limited to HLA-B27 haplotypes, but extends to other Class I and Class II MHC molecules including A2, A9, Bw and DR1/DR2 (27). Thus a genetic component is found in almost all patients with AS, although the HLA haplotype is not the only contributor to the development of AS because about $90 \%$ of HLA-B27 positive individuals remain free of the disease (27). This suggests that there must be an environmental trigger for AS development. Nevertheless, the association of AS and HLA-B27 provide a classic example of one genetic trait conferring susceptibility to disease.

Genetic contributions to autoimmune susceptibility have been identified in almost all known autoimmune conditions. Recent studies have shown that certain HLA-DR2 and DR3 Class II haplotypes contribute to development of SLE (28). MHC associated risk factors for SLE are not limited to Class II haplotypes but extend to involve the Class III region also particularly in superkiller viralicidic activity 2-like (SKIV2L), tumor necrosis factor (TNF) and complement component C4 gene loci regions $(29,30)$. Genetic as well as animal model studies establish that HLA antigens, particularly HLADR and DQ also contribute to autoimmune susceptibility in RA and Type 1 diabetes (3133).

Advanced genetic studies in recent years have located several polymorphisms in genes outside the MHC cluster that are associated with increased incidence of autoimmunity. Linkage analysis studies identified polymorphisms associated with signal transducer and activator of transcription 4 (STAT4) in RA and SLE (34), and nucleotide binding and oligomerization domain 2 (NOD2) in Crohn's disease $(35,36)$. Genomewide association studies, possible after the complete sequencing of human genome, further enhanced the number of genes associated with autoimmune conditions. Many of these genes are involved in signaling pathways of T cell (PTPN22)(37) and B cell (BANK1, BLK)(38-40) development, or in co-stimulation signaling during antigen presentation (CTLA4, CD40)(41-43). Polymorphisms in genes involved in interferon production (IRF5, IRF8, IRAK1, and STAT4) $(44,45)$ and cell to cell communication (IL-2, IL-12A, IL-23R) (46) may influence T and B cell interaction with self-antigens in various ways and thus be associated with autoimmune disorders. Even though many genetic polymorphisms and mutations have been found to associate with autoimmune disorders, the impact of any one genetic factor on the development of autoimmunity is very modest. Only a few conditions, such as autoimmune lymphoproliferative syndrome due to mutations in fas (CD95 or Apo 1) and autoimmune polyglandular endocrinopathy with candidiasis and ectodermal dysplasia (APECED) that occur due a mutation in the Autoimmune Regulator (AIRE) gene, are examples of autoimmune disorders arising due to direct consequence of genetic defects $(47,48)$. Most of the genetic factors provide 
additional susceptibility in the run up to full blown autoimmune disorders, but their contribution to initiation of autoimmunity remains unsubstantiated. Involvement of multiple genetic components in individual autoimmune disorders and also of one genetic polymorphism in multiple autoimmune conditions points to an input from multiple genes toward the susceptibility to autoimmunity.

Mouse models of autoimmune disorders have contributed to the understanding of the complex genetic contribution to autoimmune disorders. Clear evidence of genetic involvement in autoimmune diseases came from the observation that mice of a certain genetic background, such as MRL/lpr, (NZB x NZW) F1 and NZM2410 develop autoimmune conditions mimicking human disease. Study of such mice that spontaneously develop autoimmunity narrowed the boundary of genetic intervals that contribute to breaking of tolerance in these animal models of SLE. By backcrossing NZM2410 mice to C57BL/6 mice 3 genetic intervals named Sle1, Sle2 and Sle3 were identified that contribute to disease susceptibility (49). By segregating these susceptibility loci on a non-autoimmune background important insights in the mechanisms by which they promote autoimmunity was gained. It was found that Sle1 locus predisposes for autoantibody production against nuclear antigens $(49,50)$. Genes in the Sle2 interval lower the activation threshold of B cells, resulting in a higher production of polyclonal $\operatorname{IgM}$, but not of $\operatorname{IgG}$ antibodies $(49,51)$. B cells from mice harboring the Sle2 locus were hyperresponsive to antigen challenge suggesting that this locus may contain a gene that reduces the threshold for B cell activation (51). While Sle1 and Sle2 loci influenced the effector B and T cells, the Sle3 locus on chromosome 7 predisposed to autoimmunity by increasing antigen presentation by dendritic cells (52). The antigen presenting cells from mice bearing the Sle3 locus, were more efficient at presenting antigens to $\mathrm{T}$ cells and releasing more proinflammatory cytokines as compared to APCs from normal mice (53). Though these congenic mice models have helped in understanding the role each of these genetic intervals play in autoimmunity, the actual process by which autoimmunity is initiated is far from clear.

One problem in understanding the mechanisms of autoimmunity as a result of only genetic contribution is that at times one gene may predispose to many autoimmune conditions: PTPN22, a gene involved in B and T cell signaling is associated with autoimmune thyroiditis, RA, SLE and T1D (54). STAT4 which regulates INF gamma production is involved both in SLE and RA (55). Different manifestations of a single gene defect point to additional factors involved in the etiology of autoimmune disorders over and above the genetic elements that predispose to autoimmunity. Though an underlying genetic background favors the development of autoimmunity, disease manifestations may differ as they reflect differences in the underlying mechanisms of autoimmunity. These observations coupled with the fact that not every individual inheriting disease associated genotypes eventually develops autoimmunity suggest that multiple factors, in addition to genetics, are involved in the initiation and progression of an autoimmune response. Presently, it is believed that different genetic factors lower the threshold of an individual for breaking tolerance but the initiation of an autoimmune response needs an additional trigger. It is obvious that in addition to predisposing genetic factors, environmental influences contribute to overt autoimmunity. 


\section{Environmental Factors in Autoimmunity}

Several environmental toxins and infectious agents are associated with autoimmune disorders. Classical involvement of infections in initiating an autoimmune response is seen in rheumatic fever associated carditis and Guillian-Barre Syndrome (GBS) associated polyneuropathy (56). In rheumatic fever, the immune response against the M protein of Streptococcus pyogenes cross reacts with myosin from cardiac myocytes, thus leading to myocarditis $(57,58)$. A common event that preceeds GBS is infectious diarrhea caused by Campylobacter jejuni, suggesting infection with C.jejuni as a predisposing condition for GBS. Viral infections as well as vaccination against influenza virus have also precipitated GBS, clearly pointing to the role of infections in initiating this autoimmune myelitis (59). Definite mechanisms by which infections result in GBS are not known, but it is assumed that immune response against a pathogen later re-directs itself against antigens on gangliocytes. This type of cross reactivity is similar to the crossreactivity shown by $\mathrm{M}$ protein of $S$. pyogenes and antigens on cardiac myocytes in rheumatic fever. The cross reactivity is thought to arise from "molecular mimicry" between antigens expressed on pathogen and host tissue. Several other infections agents are thought to promote autoimmunity. Epstein-Barr virus (EBV) is associated with systemic lupus and in a study it was noticed that SLE patients develop antibodies to EBV protein EBNA-1 prior to developing autoantibodies (60). It is found that antibodies to EBNA-1 cross react with self-antigen Ro, one of the many autoantigens targeted by autoantibodies (61). Molecular mimicry has been postulated to be a mechanism by which infections can cause autoimmune diseases $(62,63)$.

The environment may influence autoimmunity in ways other than infections. Exposure to chemical substances can also promote autoimmunity. Mice exposed to environmental toxins such as mercury or demethylating agents, procainamide or hydralazine develop an autoimmune response similar to that seen in SLE. The "molecular mimicry" hypothesis does not provide an explanation for chemical-induced autoimmunity and the mechanism remains poorly understood. One possible explanation is that chemicals induce covalent modifications in the host antigens that cause the immune system to recognize modified self antigens in a manner such that an immune response occurs.

\section{Post Translational Modification of Proteins: Generation of "Neo-epitopes"}

Post translational modifications (PTM) play an important role in various cellular functions ranging from cell division to cell death by rapidly altering protein structure, function and interactions with other proteins. While doing so PTMs might also change the way the immune system recognizes a particular protein by generating "NeoEpitopes". A neo-epitope is a portion of a protein that gets modified in a manner such that recognition by a lymphocyte antigen receptor is also altered. PTMs are one way neoepitope can arise. Tolerance to neo-epitopes has not been established during lymphocyte development. Such "neo-epitopes" may activate lymphocytes in a similar manner as foreign antigens. 
A variety of PTM including phosphorylation, acetylation, glycosylation, methylation and deimination have been identified on autoantigens $(64,65)$. Importantly, several of these modified autoantigens are preferentially processed, presented and recognized by the immune system contributing to the development of autoimmunity (66, 67). PTMs associated with apoptotic cell death increase the immunogenicity of self antigens and predispose to autoimmunity. Examples of apoptosis-related PTMs include phosphorylation, acetylation, ubiquitination and transgluamination of histones (68). Phosphorylation of small nuclear ribonucleoprotein (snRNP) (U1-70K) and acetylation of histone H4 (H4-8K) cause enhanced immunoreactivity with systemic lupus sera $(69,70)$. PTMs contributing to autoimmune disease are not limited to nuclear self-antigens. Hydroxylation and glycosylation of collagen result in severe disease manifestations in a collagen-induced mouse model of arthritis $(71,72)$. In addition, deiminated proteins are targets of autoantibodies in RA (73). The high specificity and sensitivity of antideiminated protein antibodies in RA led to the development of several diagnostic kits (74). Although the preferential recognition of PTMs by autoimmune sera supports the "neo-epitope" theory for initiation of autoimmunity, the lack of reactivity to these PTM in control subjects argues for effective tolerance mechanisms. So how and when these PTM affect the development of autoimmune disorders is still an open question.

\section{Cell Death, Exposure of Hidden Antigens and Defective Clearance}

The involvement of dead cell remnants in the etiology of autoimmunity was reported in 1943 by Hargraves et al. who showed that dead polymorphonuclear (PMN) leucocytes are responsible for the LE cell phenomenon (75). They found that PMNs from the bone marrow of SLE patients have phagocytosed free nuclear material that appeared to be partially "lysed," "digested" or "decondensed". This was in contrast to the phagocytosis of nuclear material observed in the "Tart" cells from healthy individuals that retain all the characteristic of nuclear staining. The degenerated nuclear material of LE cells attracted PMNs, to form rosettes. They also observed that the chemotactic attractiveness of the nuclear material was lost once it was been engulfed by the PMNs. These were the first observations to suggest that the exposure of nuclear remnants and defective clearance may constitute mechanisms for the development of autoimmunity. Haserick and Bortz found that a plasma factor, immunoglobulin, was responsible for the LE cell phenomenon (76). This heralded the discovery of a plethora of autoantibodies against the nucleus and its components (anti nuclear, anti-DNA and anti histone autoantibodies) that are found commonly in SLE patients. Although, the mechanism for the induction of these autoantibodies is not completely understood, there is evidence that membrane disintegration following cell death might make these autoantigens accessible (77-81). In accordance with this view, increased apoptotic rates for lymphocytes were observed in patients with SLE and other autoimmune disorders (82). This raises important questions like whether it is the excessive cell death in SLE which predisposes for autoimmunity. Or is the increased apoptosis seen in SLE patients a consequence of an underlying autoimmune disease process?

Evidence so far suggests that both of the above possibilities are true to some 
extent. Apoptotic mechanisms not only expose nuclear and other potential autoantigens but also enhance the immunogenicity of these autoantigens by subjecting them to various PTMs and enzyme action (83-85). Caspases, the main effectors for apoptosis, also facilitate the translocation of nuclear autoantigens to cytoplasm by cleaving the nuclear localizing signal of various proteins (86) and allow the development of apoptotic surface blebs by activating ROCK1 kinase $(81,87,88)$. With these studies, it became clear that apoptotic cells are involved in making nuclear autoantigens available to react with $\mathrm{B}$ cells or other APC. This however doesn't explain the mechanism for the initiation of the autoimmune response. A big question remains: if potential autoantigens are expressed on cells undergoing apoptosis then why do not all individuals develop an autoimmune response at some point of time?

Excessive cell death or defects in the clearance of dead cells also predispose to autoimmunity, as implied by studies of knock-out mice lacking genes involved in clearance or regulation of apoptosis (89). One of the first clues indicating that autoimmunity arises from clearance defects was provided by $\mathrm{C} 1 \mathrm{q}$ deficient mice that developed autoimmunity resembling SLE, including immune complex deposition and accumulation of apoptotic bodies in the glomeruli (90). Similarly, animals defective for other molecules involved in the clearance of dead cells such as Mer tyrosine kinase (91, 92) or milk fat globule E8 (MFG-E8) (93) developed high titres of autoantibodies and glomerulonephritis. Clearance defects are also found in SLE patients. Macrophages derived from circulating monocytes of SLE patients are small, have impaired function and die earlier than those derived from control subjects (94). Nuclear remnants of dead cells were also found to be associated with follicular dendritic cells (FDC) in the germinal center in patients with SLE (95). This highlights the defective clearance mechanisms that are prevalent in SLE patients, and links them to the persistent antinuclear B cell activation that characterizes SLE.

Though it became clear that effective clearance of dead cells is necessary for avoiding autoimmunity, researchers soon discovered that an absence of factors involved in clearing dead cells does not always lead to autoimmunity. The strongest association between autoimmunity and dead cell clearance is observed in C1q deficiency. Yet, a specific mixed genetic background - (129/O1a X C57BL/6)F2 was needed in C1q knockout mice to develop autoimmunity (90). This may be due to the redundancy of mechanisms involved in clearing dead cells and may suggest that additional mechanisms are involved in developing the manifestations of an autoimmune phenotype. Dead cell membranes lose their phospholipid asymmetry leading to the exposure of anionic phospholipids such as phosphotidylserine (PS). The exposed PS can be recognized by a number of phagocytic scavenger receptors (96) as well as many freely circulating opsonins like T-cell immunoglobulin mucin (Tim-1, Tim-4) (97), MFG-E8 (98) and Protein S (99). This redundancy means that the deficiency of one component of a clearance system can be tolerated well. Also apart from specialized phagocytic cells, such as macrophages, dead cells can be taken up and cleared by epithelial, endothelial and fibroblastic cells (100-105).

The role of apoptosis in the initiation of autoimmunity came into question when it 
was found that apoptotic cells promote secretion of anti-inflammatory factors such as IL10 and TGF- $\beta$ from resident dendritic cells. This increases the rate of dead cell clearance while maintaining immune unresponsiveness $(106,107)$. Moreover, macrophages encountering apoptotic cells respond in an anti-inflammatory and immunosuppressive way $(108,109)$. Apoptotic cells inhibit the activation of macrophages by inducing production of TGF- $\beta$, prostaglandin E2 (PGE2) and platelet activating factor (PAF) (109). While mechanisms involved in all anti-inflammatory mediator release are not fully understood, TGF- $\beta$ secretion appears to depend on PS recognition (110). In addition to the indirect suppression of macrophage activation by TGF- $\beta$ and IL-10, direct recognition of apoptotic cells by the c-mer proto-oncogene receptor tyrosine kinase (MerTK) inhibits toll like receptors (TLR) signaling by upregulating the production of suppressor of cytokine synthesis (SOCS) proteins (111). Moreover, monocytes incubated with apoptotic cells produced IL-10 in response to lipopolysaccharide (LPS) treatment indicating the dominant immunosuppression mediated by apoptotic cells (112). Apoptotic cells also suppress TNF- $\alpha$ secretion by epithelial cells in response to LPS (104). The immunosuppression by apoptotic cells can be mediated by a rapid transcriptional repression of inflammatory cytokines $(113,114)$ as well as by the inhibition of extracellular signal-regulated kinases 1 and 2 (ERK1/2) (115).

These observations, together with the fact that apoptosis is an ongoing process that is essential in development, raised the possibility that different cell death pathways elicit varied responses from the immune system. In support of this, it was shown that necrotic cells induce the production of proinflammatory cytokines by engaging TLRs (116). The plasma membrane damage in necrotic cells results in leakage of molecules that can be recognized by pattern recognition receptors (PRR). Many of these "danger" molecules (e.g. HMGB1) are capable of promoting inflammation by engaging TLRs (117). The involvement of these endogenous molecules supports the danger model of immune activation (118). Necrosis occurs commonly as a consequence of an external insult such as infections. The danger molecules, together with pathogen associated molecular patterns (PAMPs) associated with infectious agents are capable of stimulating the production of proinflammatory molecules. Thus, innate immune responses provide suitable conditions for initiation of an adaptive immune response. It is likely that the presence of self antigens in proinflammatory conditions, such as arise during infection, may predispose for the development of adaptive autoimmunity.

\section{Autoimmunity: Interplay of Innate and Adaptive Immunity}

Autoimmunity by definition is the immune response against self antigens that are recognized as if they were "foreign". In understanding autoimmunity a lot can be inferred from the way the immune system reacts against infectious agents. After an infectious agent has breached the cellular integrity of host, the damaged cells of the host alarm the locally residing macrophages to a foreign intrusion. Soluble (complement, IgM), cell surface expressed (TLR 1,2,4,5 \&6), endosomal (TLR 7,8,\&9), and cytoplasmic (NOD like receptors) PRRs recognize PAMPs that are evolutionarily conserved on infectious agents and prepare for a response against invading agent. The 
response usually consist of local infiltration by cells of innate immune system that are capable of responding to infection as well as preparing for the presentation of foreign antigens to the adaptive immune system for a vigorous and more focused response.

\section{Immune Response to Infectious Agents and Lessons for Autoimmunity}

As soon as the infectious agent or host cell damage is identified, surrounding endothelial and stromal cells release numerous cytokines such as IL-1 $\beta$, IL-8, IL-18, IL-25, IL-33 (119) as well as several alarmins such as HMGB1, heat shock protein (HSP) and uric acid $(120,121)$ that activate locally residing macrophages, recruit monocytes and neutrophils from intravascular space into the extravascular site of inflammation and promote activation of $\mathrm{B}$ and $\mathrm{T}$ cells. Macrophages and monocytic cells recruited at the site of infection are capable recognizing PAMPs and and are primed to phagocytose and kill extracellular pathogens. If infection cannot be brought under control by these mechanisms, these cells also promote processing and presentation of antigens to $\mathrm{T}$ cells that influx into the site of inflammation. Antigens from previously unencountered pathogens are engaged by $B$ and $T$ cells with assistance initially from macrophages and later by dendritic cells (122). T cells so activated further help antigen specific B cells to differentiate into antibody producing plasma cells and long lived memory B cells completing the recruitment of the adaptive arm of immunity to fight invading infection (123). If $\mathrm{B}$ and $\mathrm{T}$ cells have responded to the pathogen earlier and have generated memory cells, an effective adaptive immunity mounts quickly. In this manner, the innate immune system not only fights against the invading pathogen, but also facilitates the activation of the adaptive arm of immunity (124).

What lessons can be learnt from pathogen-immune system interactions that can help us understand autoimmunity? It can be easily deduced that adaptive autoimmune immune responses in a local inflammatory environment will follow an activation course potentially involving autoantigens that will be similar to that against foreign infectious agents. After encountering antigens B cells undergo somatic hypermutations and affinity maturation so as to produce more specific antibody response (125). Similarly, antibodies from patients with autoimmune diseases show characteristics of hypermutation and affinity maturation suggesting that autoimmunity is an antigen driven process (126). Two fundamental differences that separate autoantigens from antigens of foreign infectious agents are "inaccessibility" as most autoantigens are within membrane compartments and "lack of inflammation" at least in the initial phase. In circumstances such as cell death there is large expression of hidden autoantigens that can be equated to the initial inoculation by an invading pathogen. If activation of innate immune response occurs simultaneously the result can be development of autoimmune response against self-antigens. 


\section{Successful Adaptive Immune Response Requires Contribution from Innate Immunity}

Previously considered as two separate branches of immunity, it is now evident that innate and adaptive components of immune system interact and intricately influence the activation of each other so as to offer comprehensive protection against foreign antigens. Various PRRs on cells of the innate immune system (e.g. dendritic cells, macrophages) are instrumental in directing the $\mathrm{T}$ cell responses by increasing the presentation of peptide MHC complex as well as upregulation of co-stimulatory molecules $(127,128)$. Engagement of BCR with an antigen together with the interaction of its CD40 molecules with CD40L expressed on activated CD4+ helper T cells promotes B cell clonal expansion and differentiation into short lived plasma cells. B cell interaction with helper T cells also promotes class switch recombination of BCR receptor and affinity maturation, migration, and transition into memory cells and long lived plasma cells (129). The role of innate immunity in facilitating B cell immunity against antigen does not stop to just promoting helper $T$ cell activation. Certain populations of dendritic cells $\left(\mathrm{CD} 11 \mathrm{c}^{\mathrm{lo}}\right)$ promote activation and proliferation of Marginal Zone B cells (MZ B cells) and B1 B cells against antigens in a T cell independent manner(130).

$\mathrm{T}$ independent $\mathrm{B}$ cell responses are mediated by the release of BAFF and a proliferation inducing ligand (APRIL) secreted by activated dendritic cells, macrophages and neutrophils $(130,131)$. It is not just the innate immune system that feeds into the adaptive immune system. Antigen specific antibodies from B cells promote clearance of opsonized antigens through Fc receptor mediated phagocytosis (132). Also activated $\mathrm{T}$ cells secrete cytokines that promote activation and maturation of macrophages and dendritic cells.

\section{Components of Innate Immunity Involved in Autoimmunity and Tolerance}

Processing and presentation of antigens are not limited to foreign or infectious agents. Dendritic cells were found to take up and process self antigens, especially from dying cells (133-135). Then how is tolerance maintained against self antigens? It follows that in the absence of inflammation uptake of self antigens, mostly from apoptotic cells, is done without maturation of dendritic cells. These immature dendritic cells express low levels of MHC-peptide complex and are devoid of co-stimulatory molecules (136). In case of infectious agents, engagement of TLRs by PAMP of infectious agents induces maturation of dendritic cells (136). Maturation of dendritic cells results in increased secretion of inflammatory chemokines (137), cytokines (138) as well as several growth factors (IL-2) needed for T cell maturation (139). Formation of peptide-MHC complexes is increased (140) along with the expression of co-stimulatory molecules that results in $\mathrm{T}$ cell activation. Interaction of self-antigens with immature dendritic cells devoid of co-stimulatory signals results in deletion or anergy of self specific T cells $(141,142)$. Are mature dentritic cells that express co-stimulatory molecules capable of initiating an immune response against self antigens? With an elegant study, Probst et al found that presentation of LCMV derived peptides by steady state immature dendritic cells induced T cell anergy whereas those presented by mature 
and activated dendritic cells promoted an immune response (143). This supports the idea of PAMP or endogenous TLR ligand engagement by dendritic cells promoting autoimmune $\mathrm{T}$ cell response against self-antigen. Though specific challenge of mice transgenic for ovalbumin expression in pancreas with various TLR ligands failed to induce autoimmunity (144) indicating that perhaps local inflammatory environment is needed for successful presentation of self-antigens.

Both murine and human B cells express TLRs including TLR1, TLR2, TLR7, TLR9 and TLR10 (145) which can be activated by various PAMPs and even with some endogenous ligands. B1 and MZ B cells are particularly responsive to TLR mediated maturation, proliferation and differentiation into plasma cells that is independent of $\mathrm{T}$ cell help (146). Nuclear autoantigens associated with RNA or DNA can activate autoreactive $\mathrm{B}$ cells by TLR7/TLR9 engagement by providing the required second signal after BCR engagement and so promoting autoantibody production without $\mathrm{T}$ cell help $(147,148)$. An example of this can be seen in activation of rheumatoid factor producing B cells with endogenous DNA of immune complexes acting through simultaneous BCR and TLR engagement (148). Thus, activation of innate immunity can promote autoimmune responses from B cells by direct activation of TLRs expressed on B cells or indirectly by promoting activation of APCs. Infections increase host cell death and promote activation of innate immune system by engaging various PRRs and thus provide a unique example by which activation of innate immunity can proceed to development of autoimmunity. Several endogenous TLR ligands like fibrinogen, hyaluronate, heat shock proteins and modified low density lipoproteins have been identified as capable of activating B cells by engaging TLR receptors (149).

\section{Infections Generate an Environment Suitable for Autoimmunity}

For a long time, infections have been thought to contribute to the development of autoimmunity. Many autoimmune conditions like GBS or autoimmune carditis arise after a bout with infection. In many chronic autoimmune conditions such as SLE and RA, infections cause flairs and worsening of symptoms. There are multiple mechanisms by which infectious agents may contribute to autoimmunity.

\section{Molecular Mimicry}

Molecular mimicry suggests that some pathogens carry epitopes similar to host molecules and that these may be responsible for the development of autoimmunity. The similarity in amino acid sequence or structure between host and infectious molecules can cause the immune response against infectious agent to cross react with self. Molecular mimicry, as a mechanism of autoimmunity is most studied in cardiac autoimmune conditions. Acute cardiomyopathy of rheumatic fever after group A streptococcal pharyngitis is a classical example of molecular mimicry related autoimmunity.

Monoclonal antibodies developed from the B cells of patients with acute rheumatic fever with streptococcal pharyngitis cross react with both streptococcal M protein and also with 
cardiac myosin (150). CD4+ and CD8+ T cells isolated from a patient with rheumatic carditis have shown cross reactivity to both recombinant myosin $\mathrm{M}$ protein and human cardiac myosin, laminin and tropomyosin (151). Molecular mimicry is also seen between Coxsackievirus B (CVB) and cardiac myosin (152). It was suggested that the immune response arising against CVB3 virus cross reacts to myosin to cause myocarditis (153). In another example of molecular mimicry being involved in the development of an autoimmune disease in humans, it was found that LPS derived from C.jejuni serotypes that predispose to GBS resembles human ganglioside structure $(154,155)$. Immunization of rabbits with C. jejuni LPS caused development of flaccid paralysis similar to that seen in human GBS patients. Affected rabbits also developed antibodies against GM1 ganglioside (156). Though many such examples of cross reactivity between infectious agents and host tissue exist, molecular mimicry fails to provide an easy explanation for the development of autoimmunity. The majority of individuals who get infected with $\mathrm{C}$. jejuni do not develop flaccid paralysis. Therefore, multiple factors from both host and infectious agents must be responsible for determining whether an episode of infection will result in autoimmunity.

\section{Bystander Activation and Epitope Spreading}

Immature APCs maintain peripheral tolerance by continuously presenting self antigens to naïve $T$ cells in absence of costimulatory molecules (157). Such engagement without costimulatory signalling results in T cell anergy. In an inflammatory environment, APCs upregulate CD80/CD86 costimulatory molecules while presenting foreign derived peptides to antigen specific T cells. APCs also secrete generous amounts of cytokines (IL-2, TNF- $\alpha$ and IL-6) which can activate nearby naïve T cells with other antigen specificities independent of TCR engagement in a phenomenon called "bystander activation" $(158,159)$.

Self reactive T cells thus can get activated to respond against self antigens in an inflammatory environment. Good examples of bystander activation leading to autoimmune disorders are provided by T1D and Multiple Sclerosis (MS). In both these diseases the concordance rates in monozygotic twins are approximately $40 \%$, indicating an environmental link to the development of autoimmunity (160-162). Rubella, mumps, CVB4 and Cytomegalovirus (CMV) infections are found to associate with T1D and antibodies against pancreatic islets are found in rubella (163) and CMV (164) infected patients. Many children get rubella and mumps early in life and protective antibodies against the two persists nearly for life. As the majority of these kids do not develop diabetes, molecular mimicry by itself does not explain the association between these viral infections and T1D. Though the exact mechanism by which different viruses can cause T1D is not fully understood, bystander activation of self-reactive $\mathrm{T}$ cell clones is likely to be involved. One study found that preexisting autoreactive T cells are needed for acceleration of disease in the CVB4-infected NOD mouse, thus implying that the accelerated disease manifestation is the effect of bystander activation rather than CVB4 infection induced direct damage of the pancreas (165). Viral (and many bacterial) components can induce the release of inflammatory cytokines and up regulation of $\mathrm{MHC}$ 
class I and class II molecules on cells. In Theiler's murine encephalomyelitis virus (TMEV) induced model of MS, the reactivity against myelin epitopes does not arise due to molecular mimicry between the virus and myelin sheath. Instead, there is a temporal progression of anti-myelin reactivity from an immunodominant myelin epitope to other myelin components and blocking of costimulatory B7 CD27 prevents the onset of chronic autoimmune demyelination (166). The TMEV model of MS also gives a good example of epitope spreading, where the immune response slowly progresses from immunodominant epitope of myelin to other non immunodominant components. Persistance of viral RNA during this time suggests bystander activation as mechanism responsible for initiation of antimyelin autoimmunity.

\section{Cryptic Antigens and Epitopes}

Self antigens commonly associated with autoimmune disorders are usually hidden inside cell membranes. Most notable of these are the nuclear antigens. This has led to the hypothesis that autoimmunity arises when these membrane bound or cryptic antigens become exposed to circulating T/B cells. Cell death provides circumstances where nuclear antigens become exposed at the cell surface (80). Infections cause massive cell death as well as result in the influx of neutrophils, monocytes and lymphocytes. Thus infections provide an environment where self ignorant, non-tolerant immune cells can be activated while encountering cryptic self antigens. While this seems a simple and likely explanation for the initiation of autoimmunity against organ specific or cell sequestered cryptic self antigens, it has been found that organisms have mechanisms to avoid "immunological ignorance" during T cell development (167). With the mediation of AIRE (autoimmune regulator of gene expression) in the thymus, several organ specific antigens are expressed in the medullary region of thymus so that even organ-specific self reactive $\mathrm{T}$ cells can be eliminated from the peripheral repertoire (167). This greatly decreases and often eliminates the risk of autoimmunity towards self antigens expressed in organ specific locations. Because self reactive $\mathrm{T}$ cells are known to exist in the periphery, it can be assumed that infections can still contribute to the development of autoimmunity by inducing cell death and providing an excess of cryptic self-antigens.

Crypticity is not only associated with antigens, but also with certain epitopes within self antigens. Certain (cryptic) portions of an antigen are not recognized by $\mathrm{T}$ cells, whereas other immunodominant portions are easily recognized. This is clearly evident from studies of proteolipid protein (PLP), an autoantigen in MS. Epitope mapping studies using overlapping peptides and PLP-specific T cells from MS patients revealed two immunodominant peptides (p30-49, p180-199) such that $\mathrm{T}$ cell lines generated using these two peptides responded well to intact PLP. However, other T cells responded to different PLP peptides (p80-99, p90-109, p170-189, p260-276) but not to intact PLP (168). Even in the case of acetylcholine receptor (AChR), three peptides (p75-115, p138-167 and p309-344) generated T cell lines but these T cells did not respond to either full length or fragments of the AChR alpha chain. Interestingly one p309-344 specific T cell line responded to a trypsin treated AChR fragment (p256-366) but not to the untreated fragment (169). This gives an example of how differential 
processing of antigen by proteases can unmask cryptic epitopes on self antigens and influence the generation of immune response against self antigens. These studies however don't implicate cryptic epitopes in the etiology of autoimmune disease, as proliferation of $\mathrm{T}$ cells in response to peptides can be viewed as an in vitro artifact. Evidence that cryptic epitopes are important in manifestation of autoimmune diseases comes from Lewis rat models of uveoretinitis where disease can be induced by immunizing rats with bovine interphotoreceptor retinoid binding protein (IRBP). Apart from the full protein, the disease was also induced by immunizing rats with cryptic peptides (170) or by transferring cryptic epitope-specific T cells (171). The importance of antigen processing by APC in the response against cryptic peptides became evident as $T$ cells lines against one of the epitopes (p1158-1180) responded to IRBP treated with endopeptidases but not against intact IRBP. Effect of antigen processing or presentation on generation of cryptic peptides involved in autoimmunity is also observed when myocarditis resulted in Lewis rats by immunization with trypsin-digested but not intact, untreated porcine cardiac myosin (172).

Immunization of B10.BR mice with cryptic epitopes (p26-40, p56-70) from the D protein of small nuclear ribonucleoprotein (snRNP) induced a lupus like syndrome (173). Similarly a cryptic peptide (p104-117) from myelin proteolipid protein was able to induce experimental autoimmune encephalomyelitis (EAE) in SJL/J mice when used as an immunogen (174). Also T cells specific to p104-117 of PLP were able to introduce disease when adoptively transferred to naïve mice. Splenocytes from SJL/J mice immunized with whole PLP did not proliferate when incubated with the cryptic p104-117 peptide (174) indicating that this epitope does not result from processing of the native protein by APCs. The ability of cryptic p104-117 peptide to induce splenocyte proliferation in an antigen specific manner, when immunized in SJL/J mice, suggests that lymphocytes recognizing such self-antigen cryptic epitopes may be a part of the normal repertoire. Events, such as infection or injury, can lead to the unmasking of these cryptic epitopes for antigen processing and presentation to such self reactive lymphocytes. Unmasking of cryptic epitopes may occur as a direct consequence of infection as several infectious agents carry unique proteases. Alternatively, the host response to infection may activate many proteases. For example, neutrophils enter infected tissues and release primary, secretory and azurophilic granules rich in various proteases (Elastase, PR3 and Cathepsin G). They also kill microorganisms by a burst of reactive oxygen species which can unmask cryptic epitopes by introducing non specific nicks in host proteins.

Moreover, neutrophil activation results in PTM of host proteins that can unmask cryptic epitopes or generate "neo-epitopes". Thus, in addition to direct involvement of infectious agents, innate host responses, such as neutrophil activation, provide conditions for the activation of self reactive lymphocytes, possibly initiating autoimmune disorders.

\section{Neutrophils: First Line of Defense against Infections}

Neutrophils are the first cells to be recruited to the site of inflammation and in most instances neutrophil responses are sufficient to clear pathogens. Being the frontline warriors against infection, neutrophils are the most numerous and most reactive immune 
cells in the body. They form about $60-70 \%$ of circulating immune cells in human blood and they enter sites of inflammation in great numbers. Neutrophils employ numerous mechanisms to capture and kill microorganisms. They can either ingest a pathogen to kill it by phagocytosis or can release granules with various toxic substances for extracellular killing.

\section{Neutrophil Engagement of Infectious Agents: Mechanisms and Consequenses}

The primary role of neutrophils is to engage and eliminate invading microbial agents. For this, they employ many effective mechanisms. Neutrophils granules can be discharged as soon as an invading infectious agent is identified. The first of these granules to be emptied are the peroxidase negative gelatinase granules (secondary granules) that contain a variety of matrix metalloprotinases (MMP8, MMP9, MMP25). These proteases degrade surrounding laminins, collagen, proteoglycans and fibronectin. Matrix metalloproteinase action is important in degrading the surrounding tissue and facilitating more neutrophil recruitment. Proteases also help in collapsing the blood and lymphatic vessels, thus blocking the escape routes for the bacteria and preventing further colonization. Secondary granules contain a variety of antimicrobial (lactoferrin, lipocalin, lysozymes) and chemotactic substances such as cathelicidin-LL37. While lactoferrin and lipocalin starve the bacteria for iron, lysozyme and LL37 are microbicidal in their own right.

Depending on the infectious agent involved and severity of infection, release of gelatinase granules is followed by the release of peroxidase-positive azurophilic (primary) granules. The azurophilic granules contain potent serine proteases: cathepsin $\mathrm{G}$, neutrophil elastase, protease 3 along with azurocidin/CAP37 and myeloperoxidase (MPO) $(175,176)$. Azurocidin/CAP37, a homologue of serine proteases is potently bactericidal but lacks protease activity (177). Azurocidin is also chemoattractant to moncytes and macrophages and helps in their recruitment at the site of inflammation (178). MPO is involved in the production of numerous reactive oxygen species (ROS), mainly hypochlorous acid, which can react with amines to form bactericidal chloramines (179). The importance of ROS production by neutrophils for the elimination of infections is evident from the fact that the absence of ROS production is associated with high morbidity from severe infection (180). Azurophilic granules also contain a LPS binding bactericidal permeability increasing protein (BPI) that provides potent bactericidal activity against Gram-negative bacteria (181).

Initially thought to form part of the innate arm of immunity, it is now understood that neutrophils play an important role in shaping the adaptive immune response against invading microorganisms. Being the first cells to arrive at the site of infection, neutrophils modulate different aspect of the immune response for effective clearance of infection. There is evidence that other than mounting their own robust effector mechanisms against infectious agents, neutrophils play a role in recruitment, activation and programming of APCs (182). Apart from secreting chemoattractant chemokines, neutrophils also recruit APCs with protease specific mechanisms that are unique only to 
neutrophils (183). Cathepsin G, a protease present in neutrophil granules mediates proteolytic activation of prochemerin to chemerin, a substance that specifically attracts APCs by engaging its ChemR23 receptor (184). Neutrophils also influence differentiation of dendritic cells to activated macrophages by secreting chemokines. The ability of dendritic cells to secrete IL-12 and TNF-alpha was severely curtailed in neutrophil depleted mice infected with Toxoplasma gondi (182). Other than secretion of cytokines, IL-12 and TNF-alpha by activated neutrophils, direct engagement of DCspecific, C type lectin, DC-SIGN with Mac-1 integrin of activated neutrophils induce maturation of DC which in turn is important for inducing T cell activation and proliferation (185). In addition, neutrophils can directly promote the proliferation and maturation of B and T cells by secreting TNF-related ligand B-lymphocyte stimulator (BLyS) (186) and interferon-gamma (187).

\section{Neutrophils as Antigen Presenting Cells}

In addition to creating an environment favorable for activation of $\mathrm{B}$ and T lymphocytes, neutrophils also function as APCs in certain conditions. Treatment of neutrophil cultures with GM-CSF, INF-gamma and IL-3 induces the expression of MHC class II molecules $(188,189)$. Neutrophils cultured with GM-CSF showed de novo synthesis of MHC class II molecules, along with CD80 and CD86. When incubated with staphylococcus enterotoxin and tetanus toxoid along with $\mathrm{T}$ cells, these neutrophils induced production of IL-2 and proliferation of T cells in a MHC class II restricted manner (190). In vivo expression of MHC class II and costimulatory molecules (CD80 and CD86) were found on neutrophils from patients with Wegener's granulomatosis $(191,192)$ and also from neutrophils recovered from the inflamed joints of RA patients (193). Neutrophils from normal healthy donors did not show constitutive expression of MHC class II or co stimulatory molecules, but their expression could be induced when neutrophils were cultured with T cells or T cell derived cytokines or with GM-CSF (192). These examples provide evidence that neutrophils and $\mathrm{T}$ cells can cooperate to manifest an adaptive immune response against microbial and perhaps against self antigens too.

\section{Neutrophil Extracellular Traps: Unique Mechanism to Counter Infections}

Fully differentiated neutrophils in circulation have two different fates - either to undergo apoptosis in the absence of any stimuli or to migrate to the site of an active infection and participate in microbial killing. Phagocytosis of microbes by activated neutrophils is a known and well studied mechanism. Recently, it was observed that other than phagocytosis, neutrophils kill microorganisms by releasing chromatin and granular proteins to engage the microbes (194). These extracellar "traps" of chromatin are named neutrophil extracellular traps (NETs) and contain proteins from both primary azurophilic granules (myeloperoxidase, elastase and cathepsin G) and secondary granules (lactorferrin and gelatinase) (194). DNA and histones form the meshwork of NETs and bind to Gram-positive and Gram-negative bacteria (194) as well as fungi (195). NET formation is now considered a unique form of programmed cell death, one that is 
different from both apoptosis and necrosis (196). Unlike in apoptosis, during the early stages of NET formation, phosphatidylserine (PS) does not relocalize to the outside of plasma membrane, DNA is not condensed or fragmented (at least initially) and caspases are not activated. Necrosis differs from NET formation in keeping the nuclear and cytoplasmic compartments separate with no appreciable changes in chromatin structure. In contrast, NET formation progresses through distinct phases, where first heterochromatin decondenses followed by a disintegration of nuclear membranes allowing mixing of nuclear and cytoplasmic contents, and finally release of a chromatin meshwork interlaced with cytoplasmic granular proteins from the cell (196).

The ability of neutrophils to deploy NETs is supported by the cytoskeleton in neutrophils, as demonstrated by a reduction in NET formation following exposure to agents that depolymerzise actin and microtubule filaments (197). Apart from cytoskeleton elements, NET formation is also dependent on ROS production by nicotinamide adenine dinucleotide phosphate (NADPH) oxidase $(196,198)$. Oxidative burst is needed for NET release, as neutrophils from patients deficient in functional NADPH oxidase subunits (who suffer from chronic granulomatous disease, CGD) are unable to form NETs (196). Recently, it was discovered that Rac2, a GTPase known to interact with NADPH oxidase is needed to make NETs (199). Also involved in NET formation are two proteases, neutrophil elastase and myeloperoxidase $(200,201)$, that degrade specific histones and allow chromatin release.

NET formation seems to be a late attempt by neutrophils to clear bacteria, as treating neutrophils with DNase affects the ability of neutrophils to eliminate bacteria at later time points ( $>2$ hours) (196). At earlier time points, DNase-treated and untreated neutrophils are equally potent in clearing bacteria, suggesting that phagocytosis is the primary mechanism employed by neutrophils. Apart from being morphologically unique NET formation is also associated with a unique biochemical process -deimination of histone arginine residues to citrulline (202). Exposure of neutrophils to inflammatory stimuli induces activation of peptidylarginine deiminase IV (PAD4), the enzyme that deiminates arginine residues on histones to citrullines (202). Activation of other PAD isoforms in higher vertebrates is associated with physiological growth processes. Deimination of histones during neutrophil activation in response to inflammatory stimuli or infectious agents provides a unique example of self protein deimination in response to external factors. The mechanism and regulation of NET formation is not fully understood, but the hypercitrullination (deimination) of histones is presumed to play a role in the decondensation of chromatin for extracellular release (203). It is likely that the conversion of positively charged arginines to neutral citrullines reduces the interactions between histones and negatively charged DNA. Does deimination of histones facilitate NET formation? NET formation requires rapid decondensation of chromatin (194). The linker histone H1 keeps the chromatin compact. Deimination of core histones is known at present. Whether deimination occurs in linker histone H1, a histone subtype responsible for maintaining the higher order structure of chromatin is still to be determined (see Chapter 3). 


\section{Deimination: a Post Translational Modification during Neutrophil Activation}

During neutrophil activation, deimination of histones occurs as a result of the action of PAD4. This enzyme belongs to a family of highly conserved, $\mathrm{Ca}++$ dependent enzymes known as peptidylarginine deiminases (PAD) $(204,205)$. Deimination of proteins is notable because it transforms a positively charged amino acid (arginine) to a neutral amino acid (citrulline) resulting in a net loss of one positive charge per transformed residue. So deimination changes not only the primary sequence of a protein is but also the net charge on the protein. During this reaction, the guanidino group of arginine is attacked by the Cys residue of a PAD enzyme resulting in the formation of a tetrahedral adducts. A nucleophilic attack by a water molecule then cleaves the adduct to form a keto group of citrulline while releasing ammonia and regenerating the Cys residue of the enzyme $(206,207)$. The net result is the conversion of arginine to citrulline with a mass shift of +1 Da. PAD4 is the only member of this family that has a nuclear localization signal and is therefore responsible for deimination of histone and other nucleroproteins during neutrophil activation. Other members of the PAD family, PAD1-3 and PAD6 have other tissue localization and substrate specificities.

\section{Physiological Effects of Protein Deimination}

Deimination of proteins changes their primary, secondary or tertiary structure and also influences protein-protein interactions. Deimination may result in a loss of ordered structure and denaturation of proteins (208). Deimination by causing loss of basic residues and alterations in charge distribution results in loss of ionic interactions and breaking of hydrogen bonds. In general, deimination results in a less compact configuration of proteins. In epidermis keratin (K1 and K10), filaggrin and trichohalin are known to undergo deimination $(209,210)$. After undergoing deimination, profilaggrin acquires an open configuration that is more accessible to the neutral protease calpain. Cleaved filaggrin units are osmotically active, contributing to hydration of the skin (211). Deimination further causes small filaggrin units to bind keratin more efficiently by lowering their isoelectric point and thus providing strength to the epidermis (211). During early postnatal life, deiminated myelin basic protein binds less tightly to gangliosides and thus provides the plasticity needed for brain development (212). Without deimination development of nerve and hydration of skin suffers. Also, without deiminating histones, neutrophils do not make NETs efficiently resulting in defective bacterial clearance $(203,213)$. These examples illustrate the important role deimination plays in the development of nerves, maintenance of skin characteristics and neutrophil function.

\section{Deiminated Proteins and Autoimmunity}

Interest in deimination as a PTM that enhances and promotes autoimmunity started when it was discovered that previously known anti-keratin antibodies associated with rheumatoid arthritis actually recognize deiminated filaggrin (214). Later, 
autoantibodies against several other deiminated proteins were identified in RA patients. These were directed against deiminated fibrin, vimentin and collagen. Many of these deiminated proteins were identified in the synovial joints of RA patients $(215,216)$. Detection of anti- deiminated protein antibodies paved the way for the development of kits used for diagnosing RA with very high sensitivity and specificity (217-219). Antibodies to deiminated proteins can be identified in the sera of RA patients even before the onset of disease and have been associated with severe erosive arthritis $(220,221)$. All these findings suggest that deimination plays an important role in the etiology of RA.

In addition to RA, deimination seems important for the etiology of multiple sclerosis (MS). A higher percent of deiminated MBP is found in the nerves of children that decreases with age (212). Deimination of myelin basic protein (MBP) found in adult MS patients is comparable to early post natal levels (212). T cells that interact with deiminated MBP were recognized in MS patients (222) as well as in mouse models of MS (223). When tested, deiminated peptides of MBP induced more severe disease in EAE models of MS than unmodified peptides (223).

How can peptides with one modified residue make such an impact in inducing autoimmune disorders? Perhaps deimination of arginine results in generation of "neoepitopes" influencing antigen processing, presentation or recognition. Studies have shown that positively charged P4 pockets of certain MHC class II molecules (of HLA DR $\beta$-chain) can interact with citrullines better than arginine for antigen presentation (224). Individuals which share a common epitope (EQKRAA) in the third hypervariable region of the DRB chain of MHC molecules share increased susceptibility to RA $(225,226)$. The genetic risk for RA is not limited to MHC haplotypes bearing the shared epitope but extends to include additional MHC alleles and genes outside MHC region (227). A genetic study of Japanese patient population found that certain haplotypes of PAD4 associate with an increased susceptibility for RA (228). The study revealed that the mRNA of RA susceptible PAD4 haplotypes is 4-5 time more stable than the mRNA of non-susceptible haplotypes (228). Increased mRNA stability of PAD4 may result in more extensive deimination of proteins due to higher expression of PAD4. Other evidence which points to the role of PAD4 induced deimination in RA includes the presence of anti-PAD4 antibodies in anti CCP positive RA patients (229) and colocalization of PAD4 and deiminated proteins in RA synovial tissue $(230,231)$. Also patients with anti-PAD4 autoantibodies have a severe RA phenotype (232). Certainly autoantibodies against deiminated proteins play a role in destruction of synovial tissues and joints, but how these autoantibodies arise is not yet known.

\section{Neutrophil Activation: Confluence of Factors for Initiation of Autoimmunity against Deiminated Histones?}

The following factors are implicated in predisposing to autoimmunity: 1. Genetic background influencing immune signaling, antigen processing and presentation; 2. Excessive cell death; 3. Defects in clearance of dead cells; 4. environmental agents particularly infections and chemical pollutants; 5. Exposure of membrane bound antigens 
and 6; Post translational modifications generating 'neo-epitopes'. Autoimmunity is a multifactorial event and neutrophil activation can bring majority of above mentioned factors together resulting in autoimmunity in susceptible individuals. In addition to phagocytic response to clear infections, neutrophils employ NETs with extruded chromatin laced with deiminated histones to contain infectious agents. Death of neutrophils during NET formation adds to increased cell death seen during infection and may overwhelm local clearance mechanisms. NETs also cause contact activation of plasma kallikrein (PK) of intrinsic coagulation pathway (233). On one hand this results in coagulation of clotting factors thereby augmenting microbial trapping while on the other hand it results in the release of bradykinin, a potent proinflammatory peptide (234). This along with the inflammatory cytokines released by neutrophils, resident macrophages and epithelial cells generates a highly proinflammatory microenvironment. Thus, neutrophil activation and NET formation increase the local concentration of chemotactic and stimulatory cytokines that further enhance the infiltration of $\mathrm{B}$ and $\mathrm{T}$ lymphocytes at the site of infection. Adjuvant effects of bacterial products such as LPS can only increase the risk of initiating an unwanted immune response against NET associated proteins.

Autoantibody targets on neutrophils were identified in SLE, small vessel vasculitides and many of these targeted antigens are integral components of NETs (235238). Autoantibodies have been identified against neutrophil proteases- neutrophil elastase, cathepsin $\mathrm{G}$ and proteinase 3 (PR3) $(235,236)$ as well as other bactericidal substances, such as defensins and the LL-37 peptide $(239,240)$. In SLE patients neutrophils are reported to have increased propensity to make NETs (241). These patients also have increased serum levels of NETs and associated granular proteins (242) as well as autoantibodies against them (243). Both levels of NET associated granular proteins and autoantibodies against them correlate well with the severity of disease. Released NETcomponents in SLE patients causes direct endothelial damage as well as contributes to the production of type 1 IFNs from plasmacytoid dentritic cells $(240,242)$. The fact that anti-DNA autoantibodies of lupus patients protect NETs from being degraded by nucleases only adds to the persistence of NETs further amplifying the damage (243).

Two major components of NETs are DNA and core histones that constitute about $70 \%$ of NET associated proteins (244). Histones are also well known autoantigens in diseases like lupus (245) and Felty's Syndrome (246). Of the 4 core histones present in NETs, 3 (H2A, H3 and H4) are known substrates of PAD4. The fact that autoantibodies are found against all major NET components and that histones in NETs undergo deimination, a PTM that changes recognition of self antigens in a manner to initiate an autoimmune response, made us think that autoantibodies against deiminated histones should also arise in susceptible individuals. Therefore, we began this study to find neutrophil involvement in autoimmune diseases. 


\section{Aims and Objectives}

Infections have long been suspected to play a role in the etiology of autoimmune disorders. Though the role of infections in the initiation of autoimmune disease is not clear, evidence of flares in autoimmune disease during episodes of infection support their involvement in autoimmunity. Infections provide a unique confluence of events which may promote initiation of an autoimmune response. First, infection can cause massive recruitment and activation of neutrophils. Second, deimination a PTM known to alter self antigens to "neo-epitope" is associated with neutrophil activation. Third, NET formation brings deiminated histones to the outside from the confines of cellular membranes and exposes them directly to the antigen presenting cells. Fourth, the adjuvant effect of the trapped microbial components increases the efficiency of antigen processing and presentation. Fifth, a variety of cytokines and chemokines released from the activated neutrophils, macrophages and APCs at the site of infection recruits T and B cells, some of which may be capable of binding to deiminated histones if central tolerance against deiminated histone "neo-epitopes" is not established previously. Sixth, the lack of any known mechanism for reversing deimination combined with the fact that deiminated histones are embedded into exposed NETs encapsulating pathogen increases the possibility that deiminated proteins persist in an environment suitable for recognition by lymphocytes. Keeping these points in mind we formed our hypothesis that PTM, particularly those that are introduced in a pro-inflammatory context (deimination), are responsible for breaking tolerance.

In this study our main objective was to determine if deiminated histones are targets in autoimmune diseases. In the chapters to follow we provide evidence for autoimmune response against deiminated histones in patients with Felty's syndrome (FS) but not in SLE, RA and small vessel vasculitides (Chapter 1). Next, we identify linker histone $\mathrm{H} 1$ as a new substrate of PAD4, provide evidence for deiminated $\mathrm{H} 1$ being an autoantigen in SLE and map citrullines on H1 subtypes expressed in neutrophils (Chapter 2). Finally, we describe profound B cell tolerance to deiminated histone seen in both non-autoimmune and autoimmune prone mice (Chapter 3).

\section{Significance}

Mechanisms involved in the breaking of tolerance to self antigens and initiation of autoimmune disorders are multifactorial. Environment and infections seem to influence the development and progression of autoimmune disorders. Autoimmune disorders flair during infection indicating direct role for infectious agents in the disease progression. Mechanisms like molecular mimicry of host antigens by microbial proteins have been identified as directly contributing to autoimmunity. Though a direct contribution by infections to autoimmunity has been established in some cases, in this study we looked for evidence for indirect contribution of infections in breaking tolerance. We focused on how an immune response (neutrophil activation) initiated to fight infections may contribute to the initiation of autoimmune response. We found evidence for the presence of autoantibodies against deiminated histones in a subset of patients with FS, SLE and 
RA. In contrast, autoantibodies from patients with vasculities preferentially bound to non-deiminated histones over deiminated histones suggesting that different circumstances or genetic differences can skew the autoimmune response to one form of histones. Preferrential binding of anti-deiminated histone autoantibody containing sera to activated neutrophils and the specific recognition of deiminated histones in activated neutrophil lysates suggest involvement of neutrophils as source of deiminated histones to drive autoimmune reaction.

In order to test the contribution of deimination in initiating an immune response against self-antigens, we immunized BALB/c mice with deiminated histones. Our results show that though deiminated histones are able to induce proliferation of deiminated histone specific T cells, B cell tolerance to deiminated histones is similar to that against non-deiminated histones. In mice with defective tolerance against histones (NZB X NZW, NZM2410 and SLE congenic mice derived from them), we found that spontaneously arising autoantibodies preferred non-deiminated histones over deiminated histones. This suggests that B cell tolerance to deiminated histones is actively maintained even in autoimmune prone mice. On futher investigation we found the presence of deiminated histones in the spleen of autoimmune mice and conclude that deiminated histones, probably from activated neutrophils, are trapped in the reticulo-endothelial system of spleen (247) and help in maintaining peripheral tolerance against deiminated histones even when tolerance to non-deiminated histones is compromised.

Alternatively, the preference of B cell response for non-deiminated histones over deiminated histones may be due to differences in processing and presentation of the two antigens. Proteolytic processing of proteins is a prerequisite for MHC loading. Though many proteases of the lysosomal compartment have overlapping activity, certain proteases are specific for digesting and presenting specific proteins. For example, tetanus toxin C fragment (TTCF) is specifically cleaved by asparaginyl endopeptidase (248). Consequently, APCs treated with inhibitors of asparaginyl endopeptidase, and not other protease inhibitors, have reduced presentation of TTCF antigen (248). With regard to deiminated histones, cleavage by neutrophil proteases may shift position relative to nondeiminated histones. Neutrophils express cathepsin H (249), a lysosomal cysteine proteinase involed in antigen presentation (250). Cathepsin $\mathrm{H}$ recognizes arginine as its major cleavage site. Neutrophils also express trypsin, a protease that cleaves proteins at carboxy terminal of arginine. Thus, by deiminating arginine to citrulline, the loss of a cleavage site on histones may result in different peptide fragments. Because histones are abundant self proteins, it is not surprising that they are presented in MHC. Thus, an arginine containing peptide from histone $\mathrm{H} 2 \mathrm{~A}$ (RIIPRHLQL) has been identified as a natural endogenous ligand for HLA-G (MHC-1) (251). Deimination may change presentation of this or similar histone peptides by MHC. Differences in presentation may influence $\mathrm{T}$ cell central tolerance and $\mathrm{B}$ or $\mathrm{T}$ cell activation in the periphery. As splenocytes from (NZB X NZW) F1 mice proliferated in response to both deiminated and non-deiminated histones, we suggest that MHC presentation of deiminated histones is intact in lupus prone autoimmune mice. Therefore, the preference of IgG binding for non-deiminated histones may be due to the presence of a $\mathrm{B}$ cell tolerance mechanism to deiminated histones. 
Our finding that autoantibodies to deiminated histones are present in humans with FS represents the first instance where a product of the innate immune system is directly targeted by an autoimmune response. Lack of autoantibodies against deiminated histones in patients with vasculitis indicate that different genetic predisposition or environmental circumstances determines whether an autoimmune response against deiminated histone will be initiated or not. Our observation in autoimmune mice that peripheral tolerance to deiminated histones is intact and separate from tolerance against non-deiminated histones also points to the fact that different factors, both genetic and environmental, will determine whether an autoimmune response will be initiated against either self-antigens. Understanding tolerance against deiminated histones in autoimmune lupus prone mice may increase the possibility of tolerance being manipulated in ways so as to to put an autoimmune disease into long term remission. 


\title{
CHAPTER 2. FELTY'S SYNDROME AUTOANTIBODIES BIND TO DEIMINATED HISTONES AND NEUTROPHIL EXTRACELLULAR TRAPS*
}

\begin{abstract}
Summary
To test the hypothesis that autoantigen modifications by peptidylarginine deiminase IV (PAD4) increase immunoreactivity, sera from patients with systemic lupus erythematosus (SLE), rheumatoid arthritis (RA), Felty's syndrome (FS), and ANCAassociated vasculitides (AAV), along with non-autoimmune controls, were tested for binding to activated neutrophils, deiminated histones, and neutrophil extracellular traps (NETs). IgG binding to LPS-activated neutrophils was assessed by confocal microscopy, and binding to in vitro deiminated histones was measured by ELISA and Western blot. In addition, we quantitated histone deimination in freshly isolated neutrophils from the blood of patients. Increased IgG reactivity with activated neutrophils, particularly NETs, was paralleled by preferential ELISA binding to deiminated histones over nondeiminated histones in a majority of FS but only rare SLE and RA sera. Immunoblotting revealed autoantibody preference for deiminated histones $\mathrm{H} 3, \mathrm{H} 4$ and $\mathrm{H} 2 \mathrm{~A}$ in most FS and a subset of SLE and RA patients. In patients with AAV, serum IgG preferentially bound non-deiminated histones over deiminated histones. Increased levels of deiminated histones were detected in neutrophils of RA patients. Circulating autoantibodies in Felty's are preferentially directed against PAD4-deiminated histones and bind to activated neutrophils and NETs. Thus, increased reactivity to modified autoantigens in FS implies a direct contribution of neutrophil activation and the production of NET-associated nuclear autoantigens in the initiation or progression of FS.
\end{abstract}

\section{Introduction}

Autoimmune disorders such as systemic lupus erythematosus (SLE) or rheumatoid arthritis (RA) may progress slowly and follow a chronic path with gradual worsening of disease manifestations $(252,253)$. In other individuals, sudden flares of more intense disease manifestations may interrupt lengthy periods of symptom quiescence. One notable example of worsening in a chronically progressing disorder is provided by Felty's syndrome (FS), a variant of RA that is defined by arthritis involving axial joints, enlargement of the spleen, and a decline in neutrophil numbers (254). The decrease in neutrophil numbers is thought to be due to excessive activation of mature neutrophils and their clearance in the patient's spleen. Felty's usually arises in 1-3\% of RA patients after 10 to 15 years of fairly typical symptoms (254).

* Adapted with permission. Dwivedi, N., J. Upadhyay, I. Neeli, S. Khan, D. Pattanaik, L. Myers, et al. 2011. Felty's syndrome autoantibodies bind to deiminated histones and neutrophil extracellular traps. Arthritis Rheum (In Press). 
In an alternative view, FS does not arise as chronic progression of RA. Instead, Felty's may be closely related to a T-cell form of large granular lymphocyte leukemia (LGL) with which it shares its defining clinical features and an oligoclonal CD8+ T cell expansion (255). Due to the neutropenia, FS patients experience an elevated risk of infections. The factors determining the course of disease in any given patient are largely unknown. A prevalent finding is that autoimmune disorders may worsen in parallel with various types of infections (256), although it is difficult to separate environmental effects from underlying genetic and stochastic contributions.

Just how infections may affect autoimmune reactivity and potentially lead to sudden flares in the presentation of autoimmune disorders is not established. One possibility is that, as result of infection, the number of apoptotic cells could transiently rise because diverse pathogens induce apoptosis in infected cells (257). The increased numbers of apoptotic cells may exceed the clearance capacity of tissue-resident scavenger cells and lead to the stimulation of the immune system with antigens from the apoptotic cells $(105,258)$. This proposed mechanism is consistent with the increased risk of autoimmunity arising from genetic defects in serum factors that recognize and bind apoptotic cells or defects in phagocyte receptors that function in uptake and clearance of apoptotic cells (258). Whether inefficient clearance of cells that die from other forms of cell death also increases the risk of autoimmunity has been less thoroughly tested.

An alternative form of cell death that is induced during an infection is "NETosis". NETosis received its name from neutrophil extracellular chromatin traps (NETs) that are released in response to infectious agents ranging from bacteria to fungi (194). Once at the site of an infection, neutrophils deploy extracellular chromatin that is studded with additional bactericidal granule components and may serve to immobilize and destroy microbes $(244,259)$. The release of NETs is induced by a wide range of inflammatory stimuli (202) and depends on signals from the cell surface and the participation of the cytoskeleton (197). Autoantibodies to NET components, including lactoferrin, lysozyme and elastase $(260,261)$, arise in autoimmune disorders, suggesting that NETs should be viewed as possible stimuli for such antibodies. Nevertheless, conclusive evidence linking NETs to the induction of autoantibodies is lacking.

Core histones in NETs contain arginines that are converted to citrullines (202) by peptidylarginine deiminase 4 (PAD4), a post-translational modification (PTM) that is essential for NET release (213). PAD4 acts on various autoantigens (210, 262-266), such that antibody reactivity against citrulline-containing peptides has proven useful in the diagnosis of autoimmunity. In RA, autoantibodies react with a peptide of filaggrin, provided it contains citrulline (267), and anti-cyclic-citrullinated-peptide (anti-CCP) autoreactivity constitutes a reliable marker for this condition (217).

Because histones are the major substrates of PAD4 (268), and because antihistone antibodies are produced in SLE (245), RA (269) and FS (246), we hypothesized that, if NETs are the source of the autoantigens, autoantibodies from patients with these disorders might bind deiminated histones with preference over non-deiminated histones. 
Our results with FS sera support this hypothesis: By immunofluorescence, ELISA, and Western blotting, we found preferential binding to deiminated histones $\mathrm{H} 3, \mathrm{H} 4, \mathrm{H} 2 \mathrm{~A}$ or H1. In comparison, only few RA and SLE sera showed preferential binding to deiminated histones. A control group of patients with anti-neutrophil cytoplasmic autoantibody (ANCA)-associated vasculitides (AAV) expressed antibodies with preference for non-deiminated histones over deiminated histones. We propose that activated neutrophils contribute as sources of deiminated autoantigens to the pathogenesis of FS.

\section{Patients and Methods}

\section{Patient Sera}

Sera were collected at the University of Lubeck, the Hospital for Special Surgery, from UTHSC, and from the Veterans' Administration RA (VARA) serum repository (270) and used in accord with IRB approvals from each of the participating institutions. All patients fulfilled the American College of Rheumatology classification criteria for RA (271) or SLE (272) or ANCA vasculitis (273). Felty's syndrome was clinically defined by the coexistence of RA, chronic neutropenia $(<2000 / \mu 1)$, and splenomegaly (254), except that VARA participants were classified as having FS based on the determination of an International Statistical Classification of Disease (ICD-9) code corresponding to this disorder (714.1).

\section{Neutrophil Isolation and Stimulation}

Neutrophils were isolated from healthy donor blood (Key Biologics, Memphis, $\mathrm{TN}$ ), or from patients with RA or FS, as described (202). Briefly, neutrophils were enriched using dextran sedimentation, recovered by isolymph density gradient (GallardSchlesinger, Plainview, N.Y, USA), and suspended in PBS (without $\mathrm{Ca}++/ \mathrm{Mg}++$ ) with $0.1 \%$ glucose and $0.5 \%$ heat-inactivated human serum. Neutrophils were stimulated with $100 \mathrm{ng}$ of LPS/ml of HBSS or PBS, $2 \mathrm{mM} \mathrm{Ca}++$, for 2 hours, and lysed in $2 \%$ SDS, $5 \%$ 2-mercaptoethanol and $10 \%$ glycerol in $62.5 \mathrm{mM}$ Tris, $\mathrm{pH} 6.8$. Alternatively, autoimmune or control sera were diluted to $2 \mu \mathrm{g} / \mathrm{ml}$ of IgG and used in place of LPS.

\section{Confocal Microscopy}

Purified neutrophils were allowed to settle onto poly-L-lysine-coated glass coverslips and examined as described (202). Cells were treated with LPS (100 ng/ml) for 1 hour at $37^{\circ} \mathrm{C}$, rinsed with ice-cold HBSS, and fixed with $4 \%$ paraformaldehyde in HBSS. Coverslips were incubated with patient serum $(10 \mu \mathrm{g} / \mathrm{ml} \mathrm{IgG})$ or rabbit anticitrullinated histone H3 Abs (Abcam, Cambridge, MA, Cat \# ab5103) at 1:100 dilution for 1 hour. Antibody binding was detected with goat anti-human IgG-AF647 or goat 
anti-rabbit IgG-AF488 and Sytox orange (all from Invitrogen) and analyzed by confocal microscopy.

\section{ELISA}

Calf thymus histones or recombinant human histone H3.3 (Millipore Corp., Billerica, MA), were incubated with $0.2 \mu \mathrm{M}$ recombinant PAD4 to completion of reaction as described (274). Flat bottom, 96 well microtiter plates (Immulon 4HBX) were coated with $5 \mu \mathrm{g} / \mathrm{mL}$ of deiminated or non-deiminated histones. As control, recombinant PAD4 was added to histones in the presence of EDTA to test whether binding to PAD4 confounded our ELISA readings. Blank values were determined from plates incubated with coating buffer. Plates were blocked with $2.5 \% \mathrm{BSA}$ in PBS, and sera were serially diluted from $20 \mu \mathrm{g} / \mathrm{ml}$ of IgG and incubated for 2 hour. Binding was quantified using goat anti-human IgG alkaline phosphatase (Southern Biotech, Birmingham, AL) after addition of phosphatase substrate (Sigma) and measurement of optical density. ANCA were detected by immunofluorescence or capture ELISA, as described (275). Anti-citrullinated protein antibodies (ACPA) were measured by anticitrullinated vimentin or anti-CCP ELISA, according to manufacturers' instructions.

\section{Western Blot}

Proteins were resolved by $15 \%$ SDS-PAGE and transferred to nitrocellulose. Membranes were blocked in 5\% BSA, 0.1\% Tween 20 in TBS and incubated with patient sera at $5 \mu \mathrm{g} / \mathrm{ml}$ of IgG in TBS containing $2.5 \%$ BSA, $1 \% \mathrm{NP}-40$ and $0.1 \%$ SDS for 2 hours. Anti-human IgG-HRP was used for chemiluminescence development with "Western Lightning" substrate (PerkinElmer).

\section{Statistical Analysis}

We used an unpaired, two-tailed t test to evaluate whether FS IgG had greater preference for deiminated histones than IgG from other experimental groups.

Chemiluminescence images of Western blots were scanned and the differences in binding between different sera were evaluated by paired permutation test, as described in the results. The relative abundance of deiminated histones in neutrophils ex vivo was determined by dividing the intensity of binding to deiminated histone $\mathrm{H} 3$ by the total $\mathrm{H} 3$ binding intensity. The statistical differences between the groups were computed by Mann-Whitney (Wilcoxon) Rank test. 


\section{Results}

\section{Characteristics of Patient Populations}

To analyze potential mechanisms that induce autoreactivity, we collected sera from 32 patients with SLE, 37 with RA, 23 with FS, 55 with small vessel vasculitides, along with 10 healthy controls. Patient data are given in Table 2-1. Despite the fact that SLE, RA and FS primarily affect women, patients included 67 females and 76 males who ranged from 13 to 88 years of age (avg. 62). The male predominance is because 34 of the FS and RA samples were from the VARA repository which contains mostly male patients. Disease was active in 52 of 60 RA and FS patients, whereas 22 of the SLE patients had inactive disease. In addition, our study also included 32 males and 23 females with active vasculitis, including 25 patients with WG, 15 with MPA and 15 with CSS. Most patients were treated with combinations of azathiopine, prednisolone, methotrexate and endoxan, and 10 RA or FS patients were on anti-TNF therapy (Table 2-1). Clinical assays were performed to assess the levels of anti-citrullinated protein antibodies (ACPA or anti-CCP). Most (17 of 23) FS patients and (22 of 37) RA patients had antibodies to citrullinated antigens, an indication of more rapidly progressing arthritis. In contrast, $24 \mathrm{WG}$ patients and $14 \mathrm{MPA}$ patients contained ANCA that predominantly bound PR3 or MPO respectively (Table 2-1). The clinical usefulness of these assays was recently evaluated $(275,276)$.

\section{Neutrophil Activation Increases Autoantibody Reactivity}

Neutrophils respond to bacterial breakdown products, such as LPS, and other proinflammatory stimuli by releasing granule enzymes and casting chromatin NETs (244, 259). These complex morphological and enzymatic reactions could potentially affect autoantibody binding. To test whether neutrophil activation increases autoantibody reactivity, we prepared neutrophils from healthy donors, exposed them to LPS or left them untreated, and measured binding of IgG from SLE, RA, and FS patients. Bound IgG was detected with fluorescent rabbit anti-human IgG antibodies and visualized

relative to DNA (Figure 2-1). Control serum IgG did not detectably bind to unstimulated neutrophils or to activated neutrophils (Figure 2-1B). In contrast, IgG from FS, SLE and RA patients reacted strongly to LPS-activated neutrophils (Figure 2-1D, F, H, J, L and $\mathbf{N})$, yet binding to unstimulated neutrophils remained low.

Binding of patient IgG to NETs was particularly striking. FS2 IgG binding largely overlapped with NET chromatin (Figure 2-1D), and IgG binding closely matched the lace-like distribution of dispersed nuclear DNA. A similar pattern of IgG binding was observed with the FS3 serum (Figure 2-1F). The binding of RA3 and RA4 IgG was focused into domains that mostly coincided with the location of DNA, although certain patches of chromatin remained unbound (Figure 2-1H and $\mathbf{J}$ ). Binding of SLE6 IgG was observed in close association with NETs and it extensively overlapped with the extracellular chromatin (Figure 2-1L), as did SLE7 IgG, whose binding closely matched 
Table 2-1: Characteristics of patients

\begin{tabular}{cccccccc}
\hline Parameters & SLE & RA & FS & WG & MPA & CSS & Non-AI \\
\hline Patients (n) & 32 & 37 & 23 & 25 & 15 & 15 & 10 \\
Male (n) & 3 & 18 & 17 & 16 & 7 & 9 & 8 \\
Female (n) & 29 & 19 & 6 & 9 & 8 & 6 & 2 \\
Mean Age (yrs) & 35.2 & 64.9 & 70.3 & 54.7 & 67.4 & 60.7 & 36.2 \\
Active Disease & 10 & 31 & 21 & $25^{*}$ & 15 & 15 & - \\
pANCA( $\alpha$-MPO) +ve & 1 & 1 & - & 2 & 14 & - & - \\
cANCA ( $\alpha-P R 3)+v e$ & - & 1 & 1 & 23 & - & - & - \\
ACPA +ve & 2 & 22 & 17 & 1 & - & - & - \\
Azathiopine & 9 & - & - & 2 & 3 & 1 & - \\
Prednisolone & 26 & 24 & 8 & 24 & 12 & 14 & - \\
Methotrexate & 1 & 25 & 12 & 9 & 3 & 2 & - \\
Endoxan & 2 & - & - & 9 & 5 & 8 & - \\
Hydroxychloroquine & 17 & - & - & - & - & - & - \\
Mycophenlate & 9 & - & - & - & - & - & - \\
Anti-TNF & - & 4 & 6 & - & - & - & - \\
\hline
\end{tabular}

* Sera from the WG patients were also tested in the inactive phase of disease.

(Abbreviations: SLE, Systemic Lupus Erythematosus; RA, Rheumatoid Arthritis; FS, Felty's Syndrome; WG, Wegener's Granulomatosis; MPA, Microscopic Polyangiitis; CSS, Churg-Strauss Syndrome; Non-AI, Non-Autoimmune.) 
Figure 2-1: Binding of autoimmune sera to activated neutrophils

Autoimmune or control IgG bound to unstimulated or LPS-stimulated neutrophils (A-N). Representative sera shown are: Control serum (CS; panels A and B), FS2 (C and D), FS3 (E and F), RA3 (G and H), RA4 (I and J), SLE6 (K and L), SLE7 (M and N). Stimulated neutrophils, having released NETs, react more strongly with autoimmune IgG.

Anti-human IgG alone did not bind (O). Stimulated neutrophils reacted with antideiminated histone $\mathrm{H} 3$ (green fluorescence in $\mathrm{P}$ ). Anti-IgG binding is displayed in red and DNA in blue. 


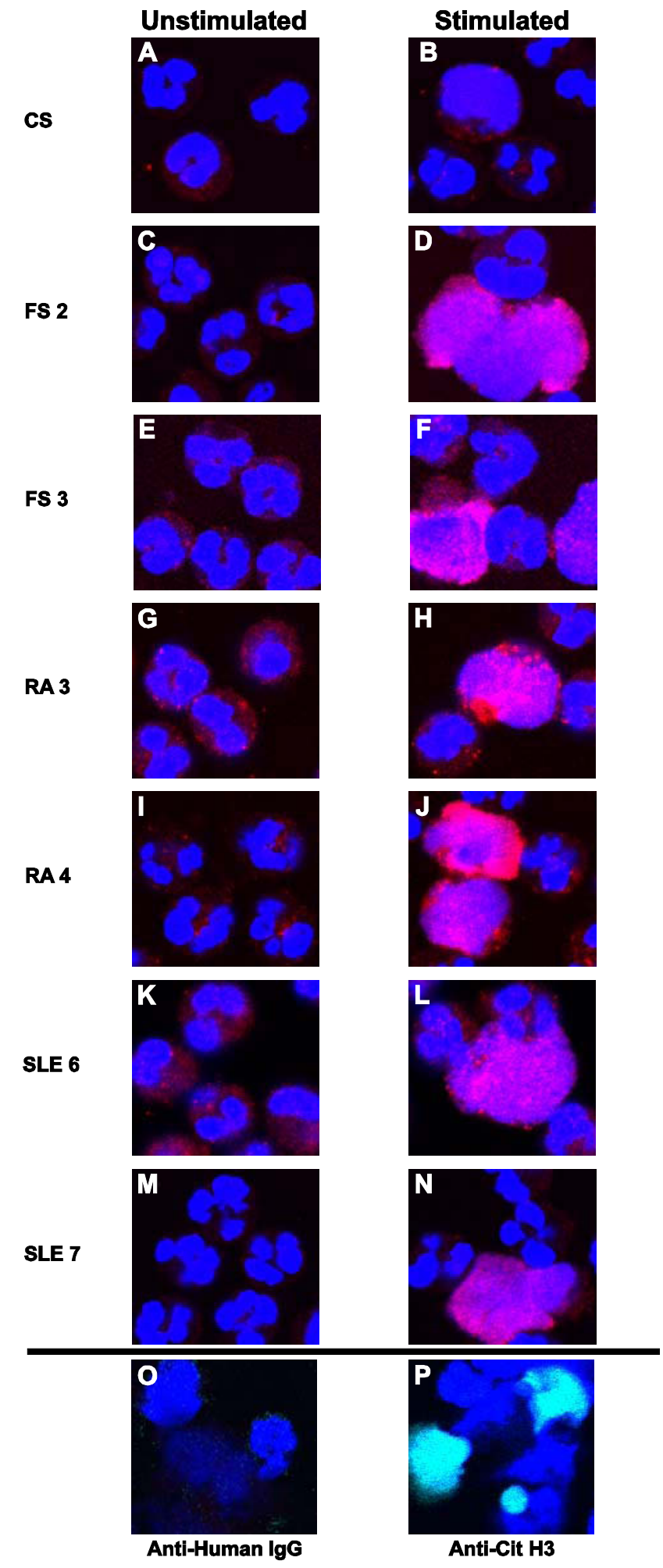


the distribution of NET DNA (Figure 2-1H). The pattern of patient IgG binding resembled, in most instances, the distribution of deiminated histone H3 (Figure 2-1P). The congruent distributions of IgG immunoreactivity and of deiminated H3 suggested that, at least in part, immunoreactivity may be enhanced by histone deimination.

\section{Preferential Antibody Binding to Deiminated Histones in ELISA}

Previous reports had indicated that neutrophils efficiently deiminate core histones (268). Our own data confirmed that core histones are the major substrates of PAD4 deimination in activated neutrophils (Figure 2-2), although a number of other proteins also are PAD4 substrates. To directly assess whether deiminated histones are preferred targets for FS, RA, or SLE patient autoantibodies, we deiminated histones in vitro and measured IgG binding by ELISA.

Binding curves were determined for 23 FS, 37 RA and 32 SLE sera. Figure 2-3A-D shows representative curves. Five of six FS sera showed IgG binding to histones in ELISA, and, for each of those 5 sera, binding to deiminated histones exceeded binding to non-deiminated histones by 2 to 10 -fold (Figure 2-3A). The sixth sample showed negligible binding. In contrast, RA sera bound less avidly to histones and, except for one, the sera showed little to no preference for deiminated histones (Figure 2-3B). Histone binding was most pronounced with SLE sera, yet except for one serum (triangles in Figure 2-3C), the binding to deiminated and non-deiminated histones was comparable. In the exceptional serum, IgG binding to deiminated histones was more than 30 -fold stronger than to non-deiminated histones. Control (Non-AI) sera showed very little to no binding to either type of histone (Figure 2-3D). LG2-2, a mouse monoclonal anti-H2B (277), gave equivalent binding to either substrate, thus indicating equal coating of plates with deiminated and non-deiminated histones (Figure 2-3E).

We subtracted the binding to non-deiminated histones from the binding to deiminated histones at $6.6 \mu \mathrm{g} / \mathrm{ml} \mathrm{IgG} \mathrm{(in} \mathrm{the} \mathrm{linear} \mathrm{range} \mathrm{of} \mathrm{titration} \mathrm{curves),} \mathrm{in} \mathrm{order} \mathrm{to}$ determine the relative binding preference for deiminated histones (Figure 2-3F). This measure indicated that FS sera were unique among the sera tested, in that binding to deiminated histones was generally higher than binding to non-deiminated histones. The SLE group had one serum with greatly enhanced preference for deiminated histones, and other sera that bound with only low relative preference to deiminated histones. IgG from RA sera showed only slightly higher binding to histones than control sera and only two sera bound preferentially to deiminated histones in ELISA. These data indicate that FS patients differ significantly from RA and SLE patients in their preferential antibody binding to deiminated histones.

\section{Autoantibodies Distinguish between Different Deiminated Histones}

The ELISA with total histones revealed binding preferences that were robust and directed at native antigens. However, the ELISA may not have detected subtle 


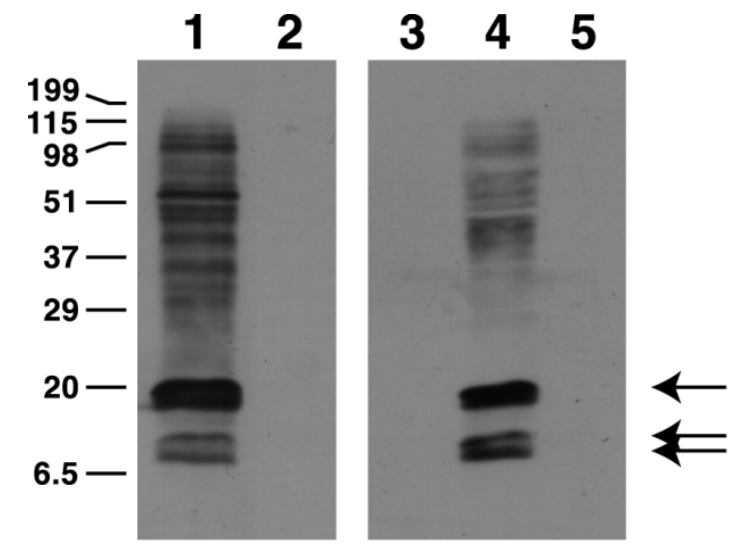

Figure 2-2: $\quad$ Neutrophil substrates of PAD4

Neutrophil extracts were probed for citrulline by Western blot. Lanes 1 and 2:

Neutrophils treated with or without calcium ionophore. Lanes 3, 4 and 5: Freeze thaw lysates of neutrophils maintained in the absence of $\mathrm{Ca}++$ (Lane 3), in the presence of $2 \mathrm{mM} \mathrm{Ca}++$ (Lane 4$)$, or in the presence of $2 \mathrm{mM} \mathrm{Ca}++$ with added Cl-amidine, a PAD4 inhibitor. Arrows indicate putative core histone bands that exceed multiple other deiminated proteins in staining intensity. 

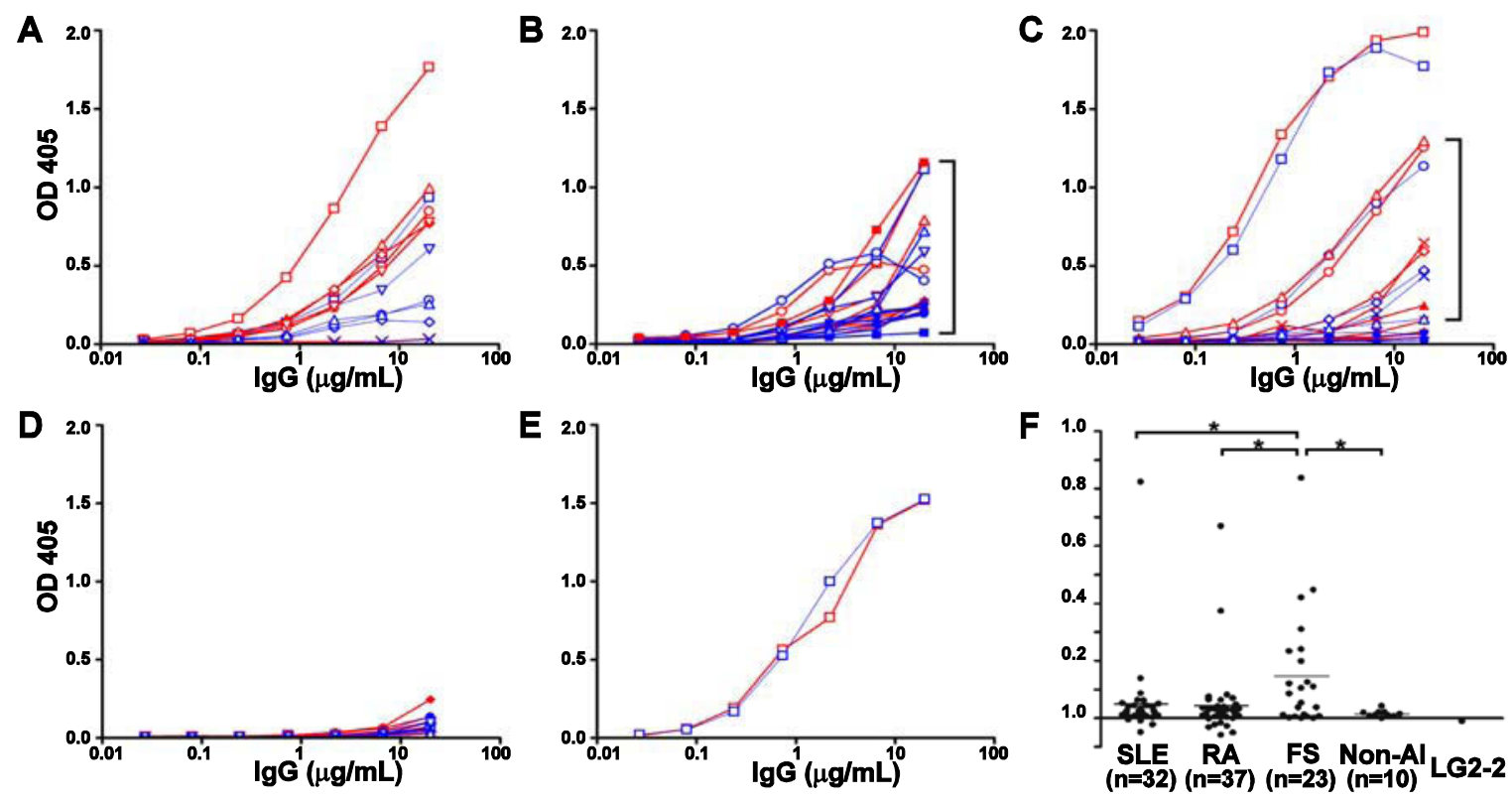

Figure 2-3: Serum IgG binding to deiminated or non-deiminated histones Sera from FS (A), RA (B), or SLE patients (C) and controls (D) were tested for binding to deiminated (red) and non-deiminated histones (blue) and OD values were plotted. Mouse anti-histone H2B (LG2-2) was used as standard (E). Differences in binding were computed for each serum (F). Different symbols represent different sera. Brackets in panels B and C identify sera with the greatest preference for deiminated histones. Asterisks indicate statistical significance between groups (FS vs. RA: $p=0.017$; FS vs. SLE: $\mathrm{p}=0.042$; and FS vs. Non AI: $\mathrm{p}=0.047$ ). 
preferences directed at individual histones. To determine which histones are preferentially bound by autoantibodies, we separated deiminated and non-deiminated histones by SDS-PAGE and probed them with patient and control sera (Figure 2-4). As shown by Coomassie Blue staining, both types of histones were present in equivalent amounts. Moreover, the faster mobility of the deiminated histones and their immunochemical reaction pattern indicated that, as expected, three core histone contained citrullines.

The majority of FS sera and a minority of the RA and SLE sera (some, e.g. RA1, quite weakly) bound with preference to one or more deiminated histones (Figure 2-4). Most of the samples that scored positive for antibodies to deiminated histones bound deiminated H3, although preferential reactivities to deiminated H2A (FS2) or H4 (SLE8) were also observed. Preferential binding to citrulline-containing epitopes from different core histones demonstrates that flanking sequences contribute to antibody binding.

Preferential binding to deiminated over non-deiminated histone $\mathrm{H} 3$ was confirmed by probing multiple gels and quantifying the statistical significance of binding (Table 2-2). In short, chemiluminescence images of Western blots were scanned and analyzed using Photoshop imaging software. Western blots were repeated at least 4 times for each patient serum. Different exposures were developed in order to establish the saturation range of the x-ray film. Films were scanned under constant settings of brightness/contrast in all cases. Band intensities were converted to numerical values using the histogram function of Photoshop. These numerical values were used to compare binding to the following pairs of histones: deiminated vs. non-deiminated $\mathrm{H} 3$, and deiminated $\mathrm{H} 3$ vs. deiminated $\mathrm{H} 4$. P values were computed to ascertain differences in $\mathrm{Ab}$ binding to different proteins by paired permutation (exact) test using R Software (online at http://www.R-project.org). Since permutation tests are based on randomly generated permutations, the $p$ values for each data set were computed 100 times and the means of all the $p$ values were tabulated. Differences were taken as significant if the $p$ value was less than 0.01 .

Interestingly, four sera (FS2, RA8, SLE5 and SLE6) preferentially bound to a deiminated protein migrating near $35 \mathrm{kD}$. The anti-citrulline antibody (Anti-Cit) detected a band migrating near $35 \mathrm{kD}$, the approximate size of linker histone $\mathrm{H} 1$, suggesting that histone $\mathrm{H} 1$ is recognized by a variety of patient sera. Although it will be necessary to conclusively demonstrate that $\mathrm{H} 1$ is a substrate for PAD4, the possibility is intriguing because $\mathrm{H} 1$ controls the higher order structure of chromatin. The analysis of patient sera by Western blot confirmed the striking preference of FS antibodies for deiminated histones and identified histone epitopes that may be particularly autoreactive.

\section{Autoantibodies from ANCA-associated Vasculitides Prefer Non-deiminated Histones}

As a control, we analyzed anti-histone autoantibodies in autoimmune disorders characterized by anti-neutrophil immunoreactivity. Wegener's granulomatosis (WG), 


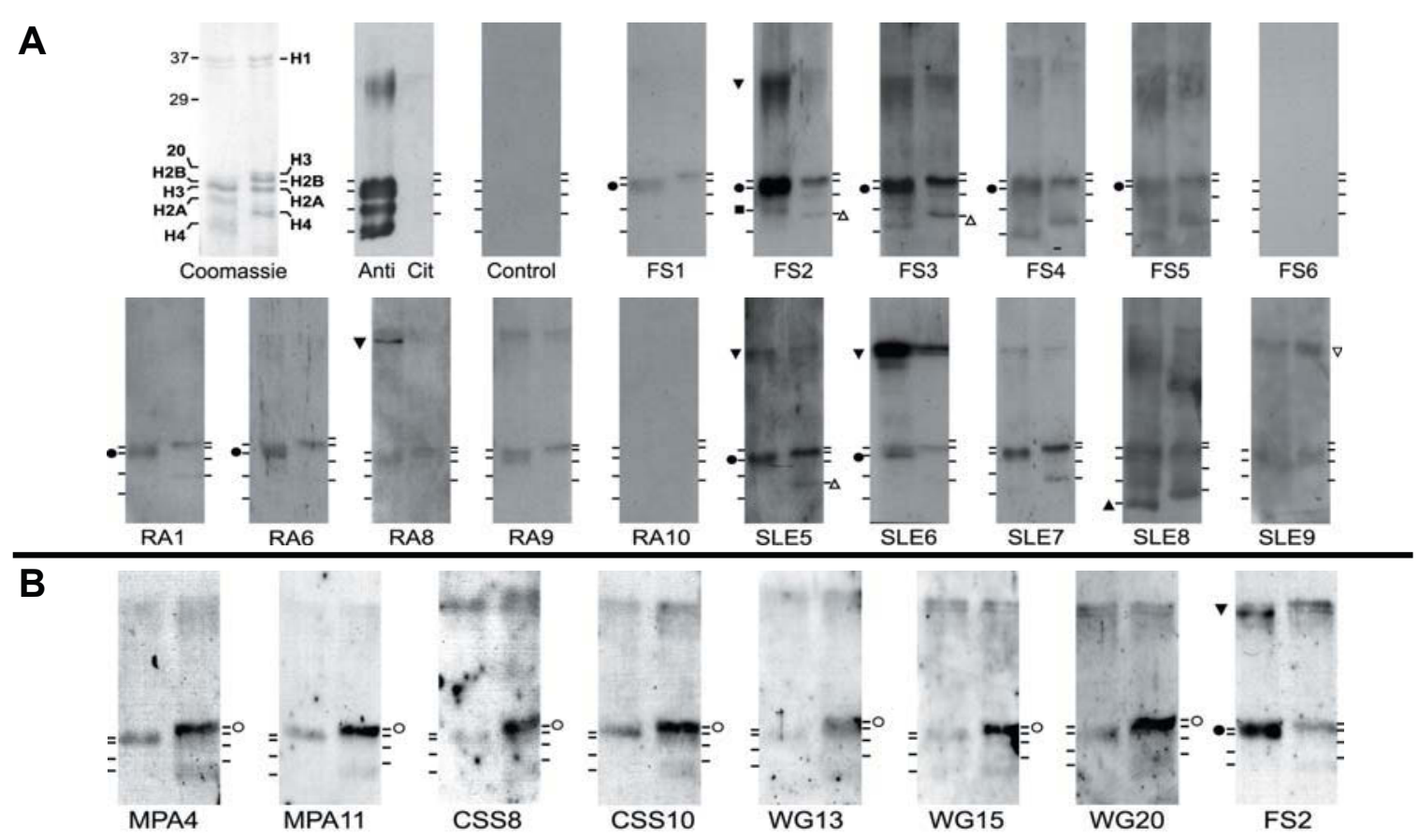

Figure 2-4: Autoantibodies distinguish different histones

(A) Representative SLE, RA, and FS sera exhibit different preferences for individual deiminated or non-deiminated histones. Deiminated (left lanes) or non-deiminated histones (right lanes) were resolved on SDS-PAGE and stained (Coomassie) or reacted with anti-citrulline (Anti-Cit) on blots. Binding of IgG from autoimmune patients (FS, RA or SLE) to histones was detected by Western blot. (B) AAV sera preferentially recognize non-deiminated histones. Western blots were performed with sera (2 MPA, 2 $\mathrm{CSS}$ and $3 \mathrm{WG}$ ) that bound histones. FS2 serum was used as positive control. Increased binding to individual histones is marked by symbols. Filled symbols indicate preference for deiminated histones, open symbols preference for non-deiminated histones. Circles denote histone $\mathrm{H} 3$, squares $\mathrm{H} 2 \mathrm{~A}$, up triangles $\mathrm{H} 4$, and down triangles histone $\mathrm{H} 1$. 
Table 2-2: Statistical analysis of IgG binding to histones

\begin{tabular}{|c|c|c|c|c|}
\hline \multirow[b]{2}{*}{ Disease } & \multirow[b]{2}{*}{ Patient } & \multirow[b]{2}{*}{$(\mathrm{n})$} & \multicolumn{2}{|c|}{ p Value } \\
\hline & & & Cit $\mathrm{H} 3$ and $\mathrm{H} 3$ & Cit H3 and Cit H4 \\
\hline Control & $\mathrm{CN}$ & 8 & 0.055015 & 0.14042 \\
\hline \multirow[t]{6}{*}{ Felty } & FS1 & 8 & 0.00783 & 0.00775 \\
\hline & FS2 & 10 & 0.001745 & 0.00189 \\
\hline & FS3 & 9 & 0.003945 & 0.003785 \\
\hline & FS4 & 8 & 0.007955 & 0.00749 \\
\hline & FS5 & 8 & 0.00777 & 0.007805 \\
\hline & FS6 & NA & NA & NA \\
\hline \multirow[t]{10}{*}{ RA } & RA1 & 9 & 0.003885 & 0.00387 \\
\hline & RA2 & 11 & 0.000865 & 0.00094 \\
\hline & RA3 & 10 & 0.45125 & 0.51419 \\
\hline & RA4 & 9 & 0.003635 & 0.00374 \\
\hline & RA5 & 11 & 0.40277 & 0.002895 \\
\hline & RA6 & 10 & 0.00185 & 0.031205 \\
\hline & RA7 & 9 & 0.78527 & 0.38305 \\
\hline & RA8 & 8 & 0.60146 & 0.007595 \\
\hline & RA9 & 11 & 0.022915 & 0.000885 \\
\hline & RA10 & NA & NA & NA \\
\hline \multirow[t]{10}{*}{ SLE } & SLE1 & 9 & 0.00419 & 0.0039 \\
\hline & SLE2 & 8 & 0.09374 & 0.054245 \\
\hline & SLE3 & 9 & 0.16782 & 0.00758 \\
\hline & SLE4 & NA & NA & NA \\
\hline & SLE5 & 8 & 0.007865 & 0.00782 \\
\hline & SLE6 & 8 & 0.007935 & 0.00807 \\
\hline & SLE7 & 9 & 0.015795 & 0.00378 \\
\hline & SLE8 & 11 & 0.1299 & 0.004625 \\
\hline & SLE9 & 8 & 0.03158 & 0.03125 \\
\hline & SLE10 & & NA & NA \\
\hline
\end{tabular}

Paired permutation test was used to calculate $p$ values for the identity in binding to deiminated and unmodified histones. Comparisons were made between deiminated H3 and unmodified $\mathrm{H} 3$, and between deiminated $\mathrm{H} 3$ and deiminated $\mathrm{H} 4$. Values of less than 0.01 were considered significant (bold). The number of observations is indicated by (n). 
microscopic polyangitis (MPA) and Churg Strauss syndrome (CSS) constitute a group of vasculitis disorders associated with ANCA (278). The characteristics of 25 patients with WG, 15 patients with MPA and 15 patients with CSS vasculitis, including their autoantibody profiles and therapy, are shown in Table 2-1. By ELISA, only 7 AAV patient sera bound to histones (data not shown). When those sera were tested by Western blot, all 7 AAV sera preferentially bound non-deiminated histones over deiminated histones (Figure 2-4). As before, the FS2 serum preferentially bound deiminated histones. These results showed that AAV patients overall have a low incidence of antihistone autoantibodies and that binding of those antibodies is inhibited by deimination.

\section{LPS Stimulation of Neutrophils Generates Preferred Histone Autoepitopes}

The preceding experiments demonstrated that in vitro deimination of histones with PAD4 affects autoreactive IgG binding. To test whether neutrophil activation also generates autoepitopes that mediate autoreactive $\mathrm{IgG}$ binding, we exposed neutrophils to LPS for 2 hours and probed blots of neutrophil lysates with FS2 and a control antiserum (Figure 2-5). Indeed, the FS2 antibodies showed preferential binding to a protein migrating with the expected mobility of deiminated histone $\mathrm{H} 3$, providing evidence that the relevant FS2 IgG histone autoepitopes are generated by neutrophil exposure to inflammatory stimuli. In addition, other proteins were immunoreactive, and LPS stimulation broadly increased the binding of FS2 IgG to cell extracts (Figure 2-5A). These results were consistent with the induction of immunoreactive epitopes by PAD4 activation in neutrophils.

\section{Deimination Is Responsible for Preferential Histone Binding}

Histones accept numerous post-translational modifications (PTM) that, in various combinations, constitute a "histone code" (279). In order to exclude possible contributions of other PTM to the preferential binding of autoantibodies, we used histone H3.3 produced in E. coli and thus devoid of eukaryotic PTM. We deiminated H3.3 with PAD4 in vitro. In parallel, we deiminated BSA to test IgG cross-reactivity with citrullines in an unrelated polypeptide.

By Western blotting, FS2 IgG bound with strong preference to the deiminated histone H3.3 over the non-deiminated histone. This result indicated that citrulline is a major determinant of FS2 IgG binding. Furthermore, a low level of binding to deiminated BSA was also observed (Figure 2-5B), suggesting that binding can accomodate some degree of heterogeneity in flanking sequences.

To localize FS2 IgG binding relative to deiminated histone $\mathrm{H} 3$, we stimulated neutrophils with LPS on coverslips and incubated them with FS2 serum dilutions and rabbit anti-deiminated histone $\mathrm{H} 3$. Binding was visualized with anti-human IgG and antirabbit IgG antibodies (red vs. green in Figure 2-5C). The FS and rabbit antibodies were detected along NET chromatin that was visualized with Sytox orange. The extent of 
Figure 2-5: FS2 IgG prefer activated neutrophils, deiminated recombinant histone H3.3, and NETs

(A) Equal amounts of proteins from unstimulated (U) or LPS-stimulated (S) neutrophils were stained by Coomasie, or probed with FS2, or control sera. Protein of histone H3 mass (arrow) was more reactive with FS2 IgG following LPS treatment. Control serum (CS) did not bind. (B) Non-deiminated (H) or deiminated (C) H3.3 and BSA were probed on Western with FS2 IgG. LPS-stimulated neutrophils were reacted with FS2 (C) or control sera (D). IgG binding is shown in red, DNA in blue and Anti-Cit H3 in green. 

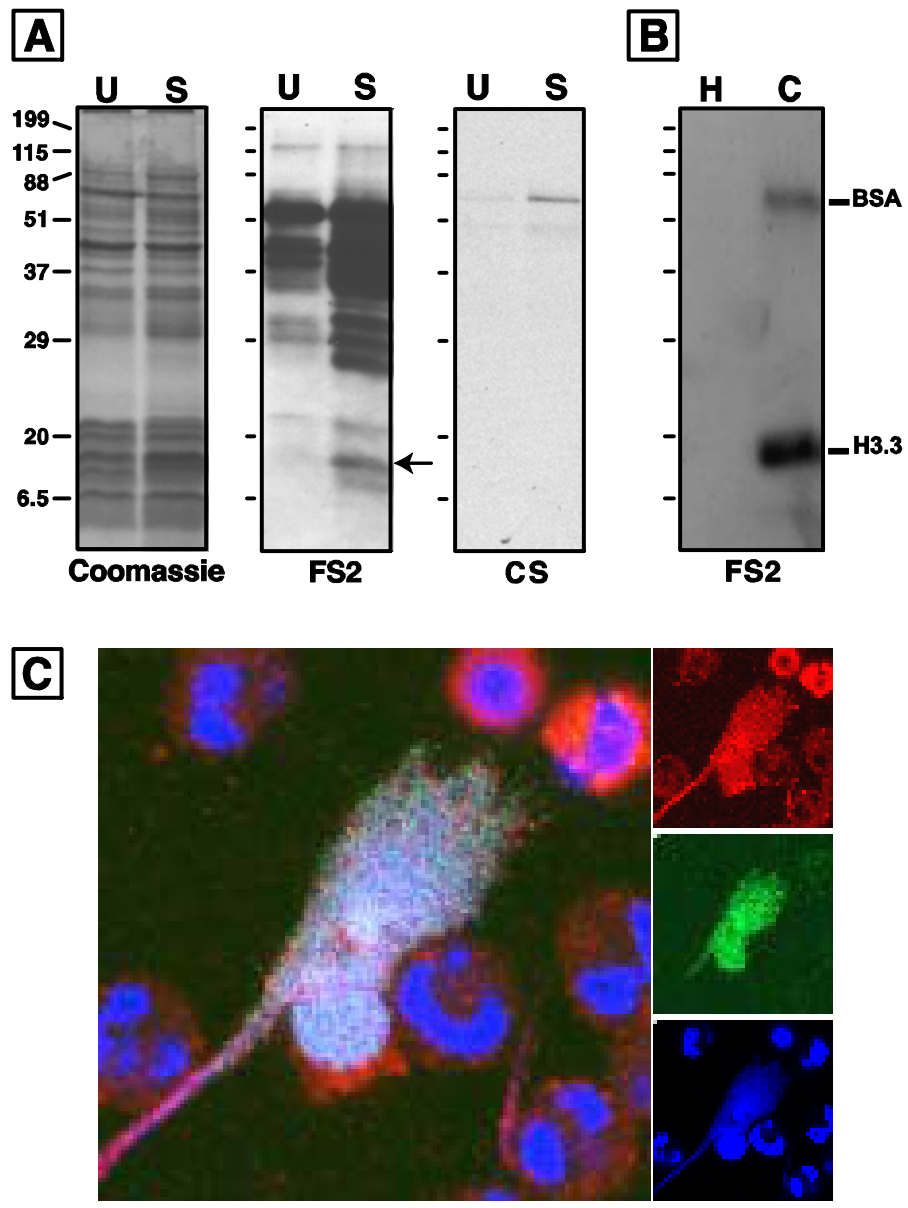

FS2

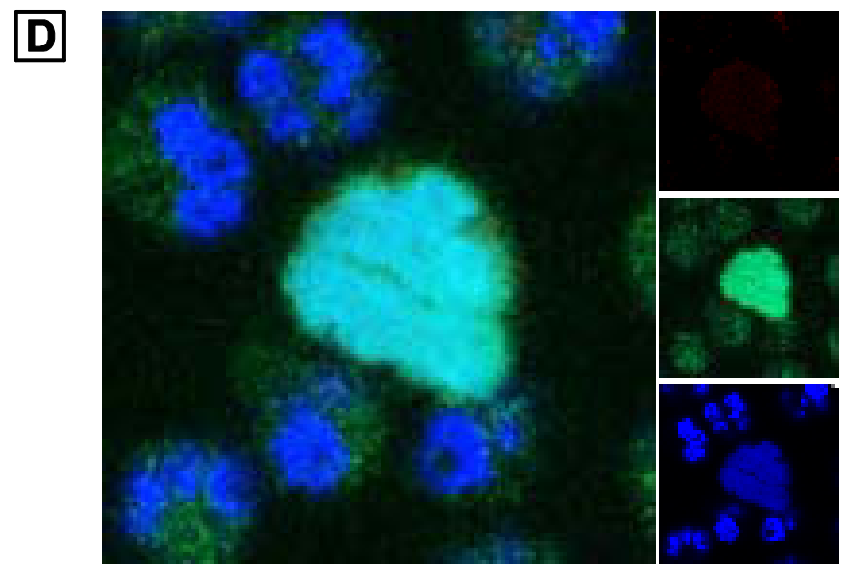

CS 
colocalization between the antibodies and DNA was nearly complete, revealing clusters of FS2 IgG along the extended NET chromatin fibers (bottom left of Figure 2-5C). This confirmed that FS2 serum contains antibodies that recognize deiminated $\mathrm{H} 3$ and other, more discontinuously arranged NET components. FS2 IgG also bound neutrophils prior to the release of nuclear chromatin, suggesting that autoantigen PTM occur in cytoplasmic foci of LPS-activated neutrophils.

\section{Circulating Neutrophils from RA Patients Show Spontaneous Activation}

To assess histone deimination in circulating neutrophils from RA, FS and control subjects, we isolated neutrophils and probed them for deiminated histone $\mathrm{H} 3$ by Western blot. Deiminated H3 signal intensities were normalized relative to total H3. The relative intensities showed a 2.9-fold higher level of $\mathrm{H} 3$ deimination in RA neutrophils over controls (Figure 2-6A; $\mathbf{p}<0.008$ ). The increased levels of deiminated histones in circulating neutrophils from RA patients suggest a greater degree of PAD4 activation. In contrast, we observed no significant increase in histone deimination in FS over control neutrophils $(\mathrm{p}>0.1)$.

To explore whether sera from FS, RA or SLE patients contain substances that activate neutrophils, we incubated neutrophils from healthy donors with $2 \mu \mathrm{g} / \mathrm{ml}$ of patient sera and observed histone $\mathrm{H} 3$ deimination at 2 hours of incubation. Both SLE and FS sera induced $\mathrm{H} 3$ deimination, suggesting the presence of immune complexes or cytokines that activate neutrophils, whereas RA sera were inactive as inducers of PAD4 in neutrophils (Figure 2-6B). Control sera showed low or undetectable H3 deimination.

\section{Discussion}

Autoantibodies target diverse PTM in autoantigens (67, 280-282), yet few PTM are uniquely associated with infections and inflammation. The exception is histone deimination by PAD4. A variety of inflammatory stimuli induce histone deimination in neutrophils, whereas apoptosis suppresses deimination (202). Here, we demonstrate that autoantibodies to deiminated histones are prevalent in patients with FS. In contrast, autoantibodies to deiminated histones are rare in RA and SLE, and absent from patients with ANCA-associated vasculitides. Our results thus suggest that inflammatory activation of neutrophils plays a central role in the induction of FS.

Our observations match findings of others that NET components are antigens in autoimmunity. Autoantibodies from patients with systemic autoimmunity recognize innate immune defense proteins that associate with NETs. This is the case for the granule proteases elastase, cathepsin $\mathrm{G}$ and proteinase $3(235,236,238)$ as well as for various bactericidal peptides that are stored in granules and co-purify with NETs, such as neutrophil defensins and the LL37 peptide $(239,240)$. PAD4, the enzyme responsible for histone deimination, is an autoantigen in RA patients (283) and anti-PAD4 antibodies precede development of clinical disease and predict its severity (284). In turn, a growing 

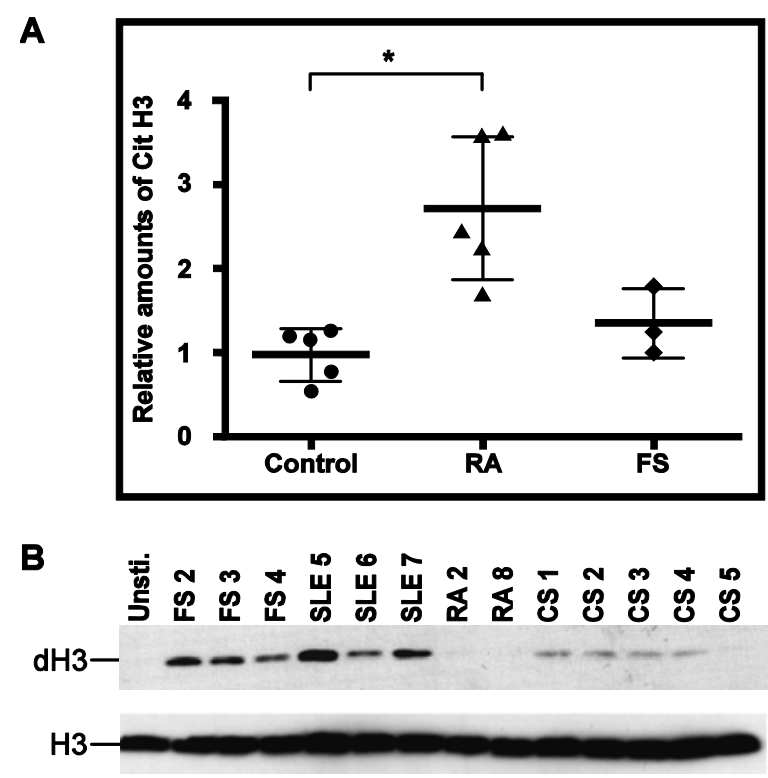

Figure 2-6: Deiminated histone $\mathrm{H3}$ in circulating neutrophils and neutrophil stimulation by autoimmune sera

Neutrophil lysates were prepared from freshly isolated neutrophils from RA, FS and control subjects. H3 deimination was determined by rabbit anti-deiminated histone $\mathrm{H} 3$ and normalized for total $\mathrm{H} 3$. The normalized amounts of deiminated $\mathrm{H} 3$ were plotted relative to the controls (set to 1). Horizontal lines represent the mean (thick line) and standard deviation (thin lines) for the groups. The difference between the RA and control groups was statistically significant $(\mathrm{p}$ value $=0.008)$ but not the difference between the FS and control groups $(\mathrm{p}>0.1)$. (B) Neutrophils were stimulated by autoimmune sera diluted to $2 \mu \mathrm{g} / \mathrm{ml}$ of $\mathrm{IgG}$ and analyzed for histone $\mathrm{H} 3$ deimination ( $\mathrm{dH} 3$ ). The blot was stripped and reprobed with rabbit anti-total $\mathrm{H} 3$. 
number of PAD4 substrates have been recognized as targets of autoantibodies. These include deiminated vimentin (264) and proteins of the extracellular matrix $(210,263$, 266), suggesting that PAD4 is active both inside and outside of cells.

How PAD4 may act on extracellular substrates became clear when it was discovered that PAD4 activation is part of the innate immune response to infections (202). Neutrophils release nuclear chromatin and associated enzymes in response to an encounter with pathogens $(194,244)$. The externalized chromatin entangles pathogens and thus becomes linked to potent adjuvants that may stimulate adaptive immunity (285). Clearly, uptake and presentation of "NET-ted" pathogens juxtaposes microbial antigens and NET autoantigens, including DNA and histones. Upon pathogen uptake and processing, self and foreign antigens may be presented to $T$ cells. The identification of autoantibodies to deiminated histones strongly suggests that NETs provide a source of autoantigens in vivo. Identification of autoantibodies to deiminated histones and other NET components $(244,260,261)$ implicates NETs as the stimulus for autoantibody production and suggests a link between infections or inflammation and the development of autoreactivity.

To directly examine the availability of autoantigens in vivo, we quantified deiminated histone $\mathrm{H} 3$ in freshly isolated blood neutrophils from RA, FS and control subjects by Western blot. We observed nearly 3-fold higher levels of deiminated histones in RA patients relative to controls (Figure 2-6A), suggesting ongoing spontaneous activation of circulating neutrophils. In FS, deiminated histones were not significantly elevated relative to controls. Because FS exhibits spontaneous activation and accelerated clearance of circulating neutrophils, our observation may be skewed by the fact that an elevated proportion of circulating neutrophils in FS is comprised of immature (PAD4-negative) neutrophils (286). Thus, the deiminated histones that were detected in FS neutrophils may have been derived from a small fraction of total neutrophils that expressed sufficiently high levels of PAD4. The observation of a potent neutrophilstimulating activity in FS and SLE sera (Figure 2-6B) supports this conclusion. Together, these results suggest the depletion of mature neutrophils from FS patients and the chronic generation of neutrophil-derived autoantigens.

In certain forms of vasculitis, neutrophils are hyperactivated and serum levels of neutrophil granule components are elevated in parallel with more advanced disease (287). In addition, the severity of lupus correlates with the levels of autoantibodies to the NETs' structural scaffold, DNA (243). Other studies directly point to neutrophil activation as a key factor in the stimulation of autoantibodies. Notably, SLE patients' neutrophils show an exacerbated response to autoantibody complexes and are more prone to NET release (241). One interesting aspect of these and our studies is that reactivities to different NET antigens correlate with different disease manifestations. Just as PR3-ANCA predominantly occur in WG and MPO-ANCA in MPA (288), the anti-deiminated histone autoantibodies associate selectively with FS. We interpret the rare development of antideiminated histone antibodies in RA as indication that tolerance to histones remains active and resilient even after development of clinical disease. Tolerance to deiminated histones may only be broken after strong stimulation of adaptive immunity by unique 
epitopes that drive both $\mathrm{B}$ and $\mathrm{T}$ cell expansion.

In closing, we propose that anti-deiminated histone autoantibodies are markers deserving particular attention in patients with severe inflammation and chronic activation of neutrophils who may be at risk of progressing to more severe disease. Chronic presentation of deiminated antigens may, over time, lead to the development of antigenspecific responses that target the modified antigen determinants and contribute to the development of other clinical features of FS. The data presented in this study allow us to propose a model of FS pathogenesis that is based on the biological properties of neutrophils, the disease manifestations of FS, and our data on the high incidence of autoantibodies to deiminated histones. In FS, pre-existing arthritis and an increased incidence of infections may stimulate a neutrophil response that includes histone deimination and the expulsion of chromatin from the cell. NETs containing deiminated histones, in complex with bacterial adjuvants, are the most likely antigenic trigger to account for the production of autoantibodies to the modified histones. These autoantibodies or their immune complexes may further stimulate neutrophils, thus completing a self-sustaining cycle that drives depletion of mature neutrophils. 


\title{
CHAPTER 3. AUTOANTIBODY RECOGNITION OF CITRULLINE IN THE GLOBULAR DOMAIN OF LINKER HISTONE H1
}

\begin{abstract}
Summary
B cells producing anti-nuclear autoantibodies require a source of antigens. Extracellular chromatin, released from activated neutrophils at sites of inflammation, may provide access to nuclear autoantigens. Deimination, a posttranslational modification of arginine to citrulline by PAD4, plays an important role in neutrophil chromatin release, and deiminated histones thus represent a mark of inflammation. Here, we identified autoantibodies in sera from systemic lupus erythematosus and Sjögren's syndrome patients that preferentially bound deiminated histone H1.2 over the unmodified H1.2. One serum bound epitope included a citrulline located at a highly conserved position in the H1.2 globular domain. We inventoried H1 histone subtypes in human neutrophils by RNA expression and by mass spectrometry. We mapped citrullines by liquid chromatography and tandem mass spectrometry. The data indicate that deimination of linker histones generates $\mathrm{H} 1$ isoforms with enhanced autoimmune potential.
\end{abstract}

\section{Introduction}

Autoantibodies in systemic autoimmune disorders such as systemic lupus erythematosus (SLE), Sjögren's disease, or scleroderma often target nuclear antigens such as DNA and histones(289-291). How such antigens engage B cells and induce autoantibodies remained unclear until circumstances were uncovered in which chromatin antigens become accessible at the cell surface $(77,292)$. Immunochemical analysis of apoptotic cells revealed that nuclear fragments bleb from the plasma membrane, thus exposing DNA and core histones to the external milieu (293). When clearance of apoptotic cells is inefficient, apoptotic blebs may supply nuclear antigens to B cells (105). However, autoantibodies to the linker histone $\mathrm{H} 1$ represented an exception to this pathway. Anti-H1 bound to early apoptotic cells but the binding to surface blebs was undetectable (293). This observation raised the question whether different circumstances are necessary for the exposure of $\mathrm{H} 1$ to the extracellular space.

In viable cells, the $\mathrm{H} 1$ linker histones bind DNA at the entry and exit points of the nucleosome core particle, thereby determining the trajectory of linker DNA that connects neighboring nucleosomes (294). By controlling the stacking of adjacent nucleosomes, $\mathrm{H} 1$ modulates overall features of the chromatin fiber. In addition, $\mathrm{H} 1$ histones control access to internucleosomal DNA, thus contributing to the regulation of gene expression (295). The involvement of H1 in the control of higher-order chromatin structure and gene transcription is reflected in the expression of multiple, structurally diverse and celltype-specific isoforms of $\mathrm{H} 1(296)$.

The capacity of $\mathrm{H} 1$ histones for chromatin condensation is modulated by a repertoire of post-translational modifications (PTM) that fine-tune $\mathrm{H} 1$ affinity for DNA 
and mediate H1 interactions with other proteins (297). Multiple H1 serine and threonine phosphorylation events correlate with mitotic chromosome condensation (298), whereas individual H1 PTM affect the interactions between H1 and RNA polymerases near active genes (299). Additional PTM in H1, including lysine methylation and acetylation, are known but their functions have not been as thoroughly examined (297).

A remarkable histone PTM that is induced in neutrophil granulocytes in response to inflammatory stimuli is the conversion of specific arginines to citrullines $(202,203)$. The conversion is mediated by peptidylarginine deiminase (PAD4) $(300,301)$, an enzyme that is required for the release of neutrophil extracellular traps (NETs) (213, 302). NETs consist of chromatin fibers that associate with components of the neutrophils' vast armamentarium of bactericidal substances (244). NETs presumably serve to enmesh bacteria and yeast pathogens and surround them with compounds that reduce their viability (194). Neutrophils from mice deficient in PAD4 are unable to produce NETs and, as consequence, the animals suffer more serious bacterial infections (213). Although core histones are prominent components of NETs, it has been controversial whether H1 histones associate with NETs $(194,244)$.

Recently, we observed that certain human autoimmune sera contain autoantibodies to deiminated core and linker histones (303). Therefore, we screened additional autoimmune sera and identified autoantibodies that recognize the deiminated human $\mathrm{H} 1.2$ histone. We used the antibodies to map the reactive $\mathrm{H} 1$ epitope and we surveyed expression of $\mathrm{H} 1$ subtypes in neutrophils by RNA expression, $\mathrm{H} 1$ purification and mass spectrometry. Purified H1 were subjected to LysC protease cleavage and citrulline mapping by MS/MS. We observed $\mathrm{H} 1$ deimination in activated neutrophils and increased immunoreactivity of one H1-reactive serum with activated neutrophils and NETs. The data argue that neutrophil activation and NET chromatin release provide circumstances for $\mathrm{H} 1$ extracellular release and stimulation of H1-reactive B cells. This observation, along with other published studies, suggests that NETs are a source of nuclear antigens that could stimulate autoreactive B cells.

\section{Materials and Methods}

\section{Patient Serum Samples}

Serum samples were collected at Institut de Biologie Moléculaire et Cellulaire; Strasbourg, France and the University of Lubeck, Germany. In all, 24 SLE, 20 Sjögren's Syndrome, 20 Scleroderma and 20 RA serum samples were tested. All patients fulfilled the American College of Rheumatology classification criteria for SLE (272), SS (304) and $\mathrm{Scl}$ (305). This study was done in accordance with the IRB approvals from the University of Tennessee, US, Institut de Biologie Moléculaire et Cellulaire; Strasbourg, France and the University of Lubeck, Germany. 


\section{In-vitro Deimination of Recombinant H1.2}

Recombinant human histone H1.2 (Axxora), were dissolved in PAD4 reaction buffer (100mM HEPES, $100 \mathrm{mM} \mathrm{NaCl}, 10 \mathrm{mM} \mathrm{CaCl} 2,0.1 \mathrm{mM}$ EDTA and $2 \mathrm{mM}$ Dithiothreitol) to a final concentration of $0.1 \mathrm{mM}$. The reaction mixture was incubated for $10 \mathrm{~min}$ at $37^{\circ} \mathrm{C}$ and then recombinant PAD4 was added to a final concentration of 0.2 $\mu \mathrm{M}$ (274). Deimination was tested by Western blot using antibody to chemically modified citrullines (Millipore) as per manufacturer's instructions.

\section{Western Blot}

For Western blot analysis, proteins were resolved on 15\% SDS-PAGE and transferred to nitrocellulose as previously described (303). Membranes were blocked in $5 \%$ BSA, $0.1 \%$ Tween 20 in TBS overnight at $4{ }^{\circ} \mathrm{C}$. Next day, the membranes were incubated with patient sera at $5 \mu \mathrm{g} / \mathrm{ml}$ of IgG in TBS containing $2.5 \%$ BSA, $1 \%$ NP-40 and $0.1 \%$ SDS using pre-blocked polyethylene pouches. After $2 \mathrm{~h}$ of incubation, membranes were washed with 1\% NP-40 in TBS. Anti-human IgG -HRP was used for detection at 1:40,000 in 0.05\% Tween 20 in TBS for $1 \mathrm{~h}$ and blots were developed using "Western Lightning" chemiluminescence reagent (PerkinElmer). For Western blots of human neutrophil extracts, cell lysates were prepared as described (202). Protein concentration of lysates was measured in a Nanodrop 1000 spectrophotometer (Thermo Scientific, Wilmington, Del., USA) and equal loading of the samples was confirmed by Coomassie blue staining. For competition assay, synthetic peptides, competitor 1 (KKAGGTP-Cit-KASGPPVS) with arginine 32 of $\mathrm{H} 1.2$ replaced with citrulline, and competitor 2 (KAVAASKE-Cit-SGVSLAA) with arginine 53 of H1.2 replaced with citrulline were kind gift from Dr. Jean-Paul Briand of Institut de Biologie Moléculaire et Cellulaire, Strasbourg, France. The peptides were incubated for $1 \mathrm{~h}$ with a dilution of SLE6 serum (5ug/ml of IgG) before the mixture was used for a Western blot.

\section{Preparation of Protease Digested Peptides}

Approximately $1.5 \mu \mathrm{g}$ of the recombinant Histone H1.2 was desalted using a 3000 kDa MW cut-off centrifugal filter device (Millipore) according to the manufacturer's instructions. Protease digestion of $\mathrm{H} 1.2$ was performed overnight at $37^{\circ} \mathrm{C}$ with $50 \mathrm{ng}$ of LysC and the digestion was stopped with the addition of TFA $(1 \%, v / v)$. The solution was dried in a Vacufuge Concentrator (Eppendorf, Westbury, NY, USA), and the dried pellet was reconstituted in $20 \mathrm{ul}$ of $0.1 \%$ TFA solution. The lysC digested peptides were loaded on a C18 Ziptip column (Millipore), washed with $0.1 \%$ TFA and eluted with $10 \mu 1$ of a $50 \%$ acetonitrile solution. The eluant was near-dried in a vacufuge, and the residue was reconstituted in $4 \mu \mathrm{l}$ of a $0.1 \%$ formic acid solution. Two $\mu 1$ of that solution were used for the LC/MSMS analysis. 


\section{Liquid Chromatography-Mass Spectrometry and Database Search}

The H1.2 peptides were resolved by HPLC by a gradient elution in which the buffer changed from solvent A $(0.1 \%$ formic acid solution $)$ to solvent $\mathrm{B}(0.1 \%$ formic acid in $90 \%$ methanol solution). Peptide masses were determined by using a Q-TOF mass spectrometer in a positive-ion mode. The nano electrospray ionization source temperature was set at $90^{\circ} \mathrm{C}$ with capillary and cone voltage set to $3.0 \mathrm{kV}$ and $30 \mathrm{~V}$ respectively. Mass calibrations were done using polyalanine (Michrom Bioresources Inc., Auburn, CA, USA). Double- and triple-charged precursor ions with a count $\geq 20$ were selected to perform MSMS. MSMS scans ( 5 second) were followed by an MS scan (1 second). Masslynx software (version 3.5) was used to process the chromatographic and mass spectrometric data. To identify proteins, Swissprot and NCBInr databases were searched using Bioworks (version 3.2; Thermo-Fisher) and Mascot (web-version) software. The precursor ion (MS) and product-ion (MSMS) mass-error tolerances were both set to $0.1 \mathrm{Da}$. The modification of arginine was set to $0.98 \mathrm{Da}$ because the monoisotopic mass difference between citrulline $\left(\mathrm{C}_{6} \mathrm{H}_{13} \mathrm{~N}_{3} \mathrm{O}_{3}\right)$ and arginine $\left(\mathrm{C}_{6} \mathrm{H}_{14} \mathrm{~N}_{4} \mathrm{O}_{2}\right)$ is $0.98 \mathrm{Da}$.

\section{RNA Extraction and Microarray Analysis}

Total mRNA was isolated from neutrophils using RNeasy Mini Kit (Qiagen) as suggested by the manufacturer. Genomic DNA was eliminated by RNase-free DNase I (Qiagen) digestion during the isolation procedure. Isolated total RNA was analyzed on an Agilent 2100 Bioanalyzer using RNA 6000 Pico Labchip Kit (Agilent Technologies). Reverse transcription to cDNA was performed at $50^{\circ} \mathrm{C}$ for $1 \mathrm{~h}$ followed by $85^{\circ} \mathrm{C}$ for 5 min with Transcriptor First Strand cDNA Synthesis Kit (Roche) using random hexamer and anchored-oligo (dT) primers (Roche). RNA quantitations were performed using the Whole-Transcript Human Gene 1.0 ST array (Affymetrix).

\section{Neutrophil Isolation and Stimulation}

Neutrophils were isolated from healthy donor blood purchased from Keybiologics (Memphis, TN) and used in accordance with protocols approved by the Institutional Review Board (IRB), University of Tennessee. Neutrophils were isolated as described (202). Briefly, neutrophils were enriched using dextran sedimentation and recovered from an isolymph density gradient (Gallard-Schlesinger, Plainview, N.Y, USA) under endotoxin-free conditions. Erythrocytes were lysed in ice-cold, hypotonic buffer $(0.2 \%$ $\mathrm{NaCl})$ for $30 \mathrm{~s}$, and lysis was stopped by adding hypertonic saline $(1.6 \% \mathrm{NaCl})$. Neutrophils were suspended in HBSS (without $\mathrm{Ca}^{++}$or $\mathrm{Mg}^{++}$) with $0.1 \%$ glucose and $0.5 \%$ heat-inactivated human serum or in HBSS with $0.5 \%$ heat-inactivated human serum at a final concentration of $2 \times 10^{6}$ neutrophils $/ \mathrm{ml}$. Neutrophils were stimulated with ionophore in $\mathrm{HBBS}$ containing $2 \mathrm{mM} \mathrm{Ca}^{++}$at $37^{\circ} \mathrm{C}$ for $2 \mathrm{~h}$. Following incubations, neutrophils were pelleted and lysed in SDS-lysis buffer (2\% SDS in $62.5 \mathrm{mM}$ Tris, $\mathrm{pH}$ 6.8 , supplemented with 5\% 2-ME and 10\% glycerol). 


\section{Linker Histone H1 Extraction and Purification}

Perchloric acid extraction of $\mathrm{H} 1$ was performed as described earlier (297).

Briefly, 50 million neutrophils were resuspended in $0.5 \mathrm{ml}$ of $5 \% \mathrm{HClO}_{4}$ and subjected to three cycles of freezing (in mix of dry ice and methanol), thawing at $37^{\circ} \mathrm{C}$ and vortexing $(10 \mathrm{~s})$. The final suspension was centrifuged at $15,000 \times \mathrm{g}$ for $10 \mathrm{~min}$. The clear supernatants were mixed with an equal volume of ice-cold $66 \%(\mathrm{v} / \mathrm{v}) \mathrm{CCl}_{3} \mathrm{COOH}$. The linker histones were precipitated for $30 \mathrm{~min}$ on ice and collected by centrifugation at $15,000 \times \mathrm{g}$ for $10 \mathrm{~min}$. The pellets were washed with $0.2 \% \mathrm{HCl}$ in acetone, then twice with pure acetone, and vacuum-dried. The dried pellets were reconstituted in high salt buffer (25mM HEPES pH 7.6 with $550 \mathrm{mM} \mathrm{NaCl}, 12.5 \mathrm{mM} \mathrm{MgCl}_{2}$ and $0.1 \mathrm{mM}$ EDTA).

\section{Confocal Microscopy}

Neutrophils were isolated as described above and allowed to settle for $30 \mathrm{~min}$ onto a poly-L-lysine coated glass coverslips as described (202). Cells were treated with LPS $(100 \mathrm{ng} / \mathrm{ml})$ for $1 \mathrm{~h}$ at $37^{\circ} \mathrm{C}$. Coverslips were washed with ice cold HBSS, cells were fixed with $4 \%$ paraformaldehyde in HBSS for 15 min at room temperature and incubated overnight with blocking buffer (HBSS with 10\% FBS, 1\% BSA, $0.05 \%$ Tween 20 and $2 \mathrm{mM}$ EDTA) at $4^{\circ} \mathrm{C}$. The coverslips were washed with wash buffer (HBSS with $3 \%$ FBS $)$ and incubated with patient serum $(20 \mu \mathrm{g} / \mathrm{ml} \mathrm{IgG)}$ or rabbit Abs that bind citrullines in the $\mathrm{N}$ terminus of histone $\mathrm{H} 3$ (Abcam, Cat \# ab5103; 1:100 dilution) in wash buffer at $4^{\circ} \mathrm{C}$ for $1 \mathrm{~h}$. Coverslips were washed and incubated with AF647conjugated goat anti-human IgG, AF488-conjugated goat anti-rabbit IgG and Sytox Orange (all from Invitrogen) for $30 \mathrm{~min}$ at $4^{\circ} \mathrm{C}$. Coverslips were washed and mounted on glass slides using mounting media (wash buffer with $50 \%$ glycerol) and analyzed by confocal microscopy.

\section{Results}

\section{Identification of Autoantibodies to Deiminated H1.2}

Previously, we identified autoantibodies to deiminated core and linker histones (303). Because deimination of linker histones has not been characterized, we screened 24 additional SLE sera, 20 Sjögren's syndrome sera, 20 scleroderma sera, and 20 rheumatoid arthritis sera for antibodies with preference for deiminated $\mathrm{H} 1$ histones (representative results are shown in Figure 3-1A). As antigen, we used recombinant human H1.2 that was treated with PAD4 in vitro or left untreated. In all, 72 sera contained anti-H1 antibodies, as assessed by Western blot, and 4 sera bound deiminated H1.2 with at least 2-fold preference over non-deiminated H1.2. However, the vast majority of patient sera showed comparable binding to either form of $\mathrm{H} 1.2$ and no serum showed a bias toward the non-deiminated H1.2. We may have missed some autoantibodies to deiminated linker histones because we did not measure binding to all 
A

Systemic Lupus Erythematosus

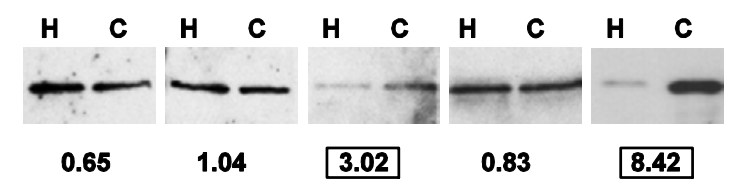

\section{Sjogren's Syndrome}

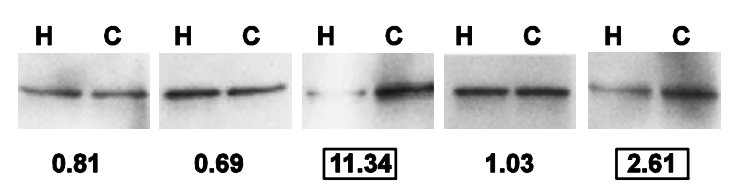

Scleroderma

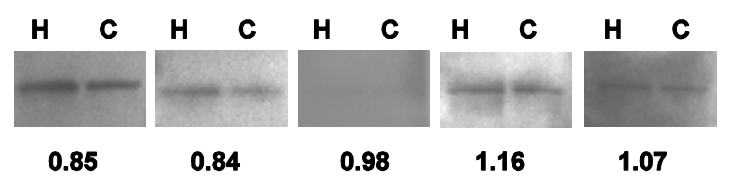

\section{Rheumatoid Arthritis}

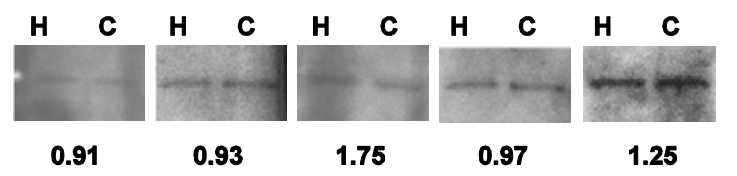

\section{B}

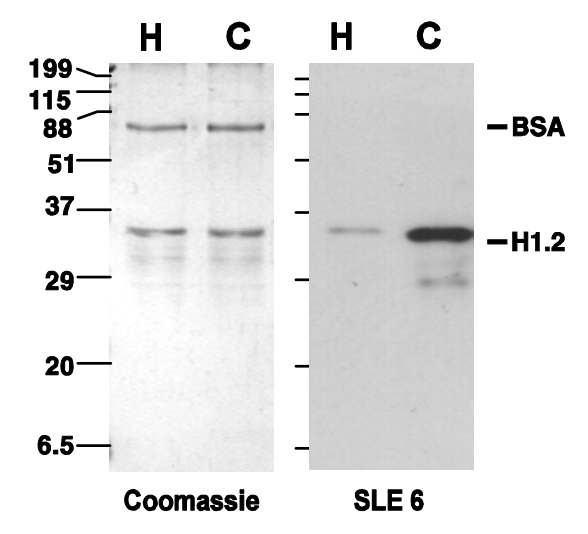

Figure 3-1: $\quad$ Preferential binding of autoimmune sera to deiminated H1.2 Equal amounts of non-deiminated $(\mathrm{H})$ and deiminated $(\mathrm{C}) \mathrm{H} 1.2$ were blotted on nitrocellulose and probed with sera from systemic lupus erythematosus, Sjögren's syndrome, scleroderma and rheumatoid arthritis patients. Five representative blots from each set are shown. The ratio of binding to deiminated vs. non-deiminated $\mathrm{H} 1.2(\mathrm{C} / \mathrm{H})$ was calculated with ImageJ software and values are listed below each blot. Two sera each of SLE and Sjögren's syndrome showed a greater than 2.0 binding preference for deiminated H1.2 (A). Equal amounts of non-deiminated $(\mathrm{H})$ and deiminated $(\mathrm{C}) \mathrm{H} 1.2$ and BSA were analyzed by coomassie staining or probed with SLE6 serum. Preferential binding of SLE6 to deiminated H1.2 over non-deiminated H1.2 and no detectable binding to deiminated BSA reveal the contribution of deiminated residue(s) and adjoining protein sequence as determinants of the specificity of SLE6 IgG (B). 
H1 subtypes whose unique epitopes may represent additional autoantibody targets (296). In the following experiments, we used the SLE6 serum (303) whose binding was highly biased toward deiminated H1.2 and did not extend to citrullines in a heterologous protein (BSA; Figure 3-1B).

\section{Determination of Autoepitope in Histone H1.2}

Because SLE6 antiserum preferentially bound deiminated histone H1.2, we asked whether one of the 3 arginines in H1.2, if converted to citrulline, formed the SLE6 binding determinant. We addressed the question by using synthetic peptides as inhibitors of antibody binding. To compete binding of SLE6 to the deiminated histone H1.2, we incubated the serum with either of two peptides (containing citrulline at position 32 or at position 53 of H1.2; Figure 3-2A) before reacting the serum against the intact histones on a Western blot (Figure 3-2B). Dilutions of the 16-mer peptides were prepared and their effect on binding to the immobilized histones was compared. The preference of SLE6 for deiminated H1.2 was maintained even after incubation with $1.0 \mathrm{ug}$ of Competitor 1 (KKAGGTP-Cit-KASGPPVS), whereas, in a parallel incubation, preferential binding was eliminated with a ten-fold lower amount of Competitor 2 (KAVAASKE-Cit-SGVSLAA). Thus, we concluded that Competitor 2, containing citrulline in place of arginine 53, was more effective as inhibitor of SLE6 binding and therefore shared structural features with the authentic H1.2 autoepitope recognized by the SLE6 serum autoantibodies.

\section{Deimination of Recombinant H1.2}

To confirm that incubation of H1.2 with PAD4 converts arginine 53 to citrulline, we subjected the deiminated H1.2 to Lys C protease cleavage and analyzed the peptides by MS/MS. We also asked if arginine 32 and 78 of H1.2 serve as substrates for PAD4 deimination. We observed multiple peptide fragments (including the $b_{2}$ and $y_{9}$ ions; Figure 3-3A) that were consistent with arginine deimination occurring within the amino terminal dipeptide of ER*SGVSLAALK. In addition, we identified citrulline at position 32 of $\mathrm{H} 1.2$ by performing MS/MS on the peptide AGGTPR*K (Figure 3-3B). The mass determination using Q-TOF spectroscopy was consistent with the presence of citrulline (1130.70 Da for the LysC-derived peptide, compared to the theoretical monoisotopic masses of 1129.65 and 1130.63 Da for the unmodified versus the deiminated peptides). To estimate the uncertainty inherent in the measurements, the experiment was performed three times and each time the error was less than $62 \mathrm{ppm}$. Thus, the mass measurements proved reliable and sufficient to discriminate between arginine and citrulline in this peptide. In contrast, we observed that the NNSRIK peptide was resistant to deimination, inasmuch as we did not detect citrulline within that peptide even after a $4 \mathrm{~h}$ incubation with recombinant PAD4 (Figure 3-3C). 


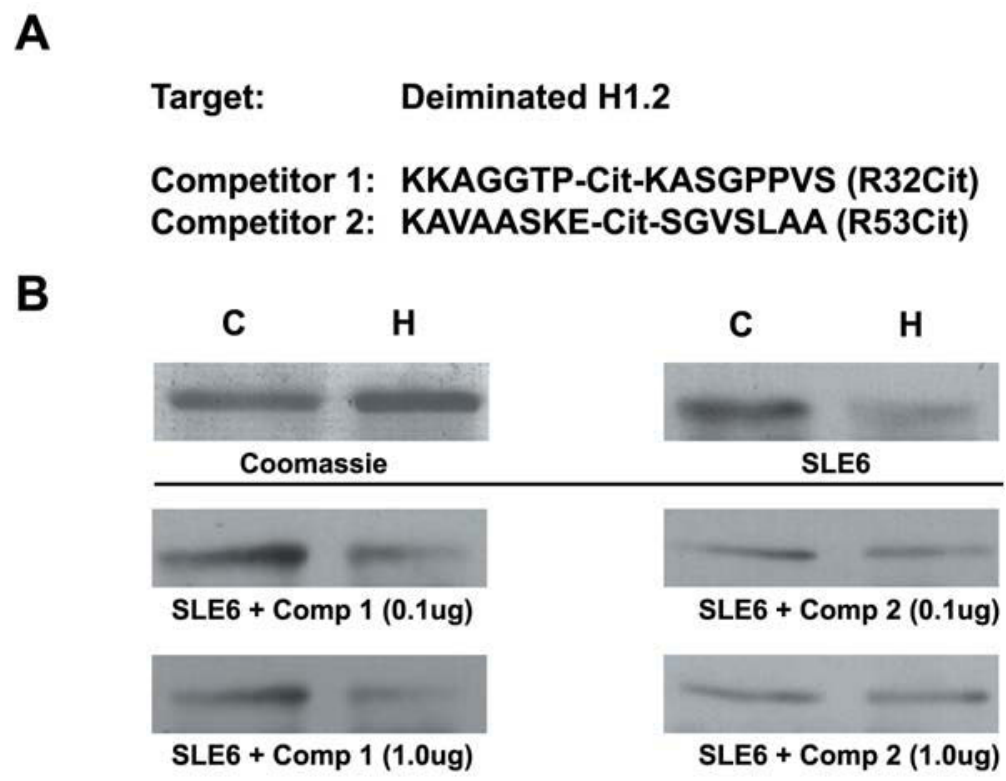

\section{Figure 3-2: $\quad$ Peptide inhibition of SLE6 binding to deiminated H1.2}

The preferential binding of SLE6 to deiminated H1.2 was assessed after a preincubation of the serum in the presence of either of two 16mer synthetic peptides (Competitor 1= R32Cit; KKAGGTP-Cit-KASGPPVS) and Competitor 2= R53Cit; KAVAASKE-CitSGVSLAA). The peptides were designed to match $\mathrm{H} 1.2$ with citrulline residues substituting for arginine 32 or 53 (A). Equal amounts of deiminated (C) and nondeiminated $(\mathrm{H}) \mathrm{H} 1.2$ were analyzed by SDS-PAGE (Coomassie) or blotted and probed with SLE6 serum. Panels below the horizontal line show results of Western blot performed following a preincubation in the presence of competitors 1 or 2 . The mass of the peptide is indicated beneath each panel. Competitor 2 (R53Cit) was more effective at blocking preferential binding of SLE6 to deiminated H1.2 (B). 


\section{Figure 3-3: $\quad$ MS/MS analysis of in vitro deiminated recombinant $\mathbf{H 1 . 2}$}

MS/MS spectra of doubly charged precursor ions of deiminated histone H1.2 treated with LysC protease to release peptides (A-C). Fragmented ions (b series and y series) are marked on respective peptide sequences and on the MS/MS spectra. Two of 3 arginines in H1.2, peptide ER*SGVSLAALK (A) and AGGTPR*K (B) showed mass consistent with deimination to citrulline. We found no evidence for peptide NNSRIK (C) deimination during the in vitro PAD4 reaction. 
A

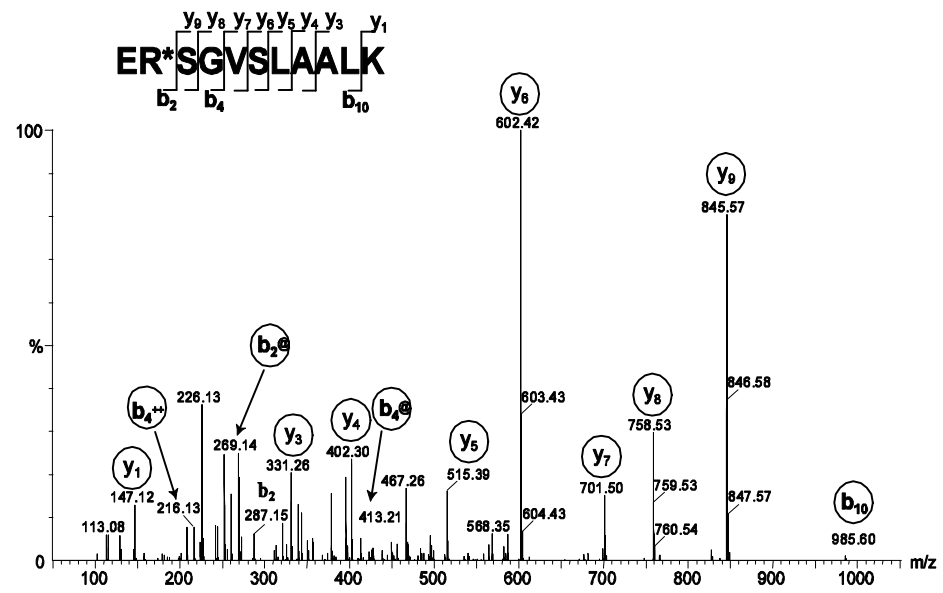

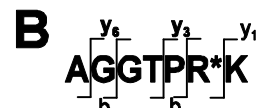

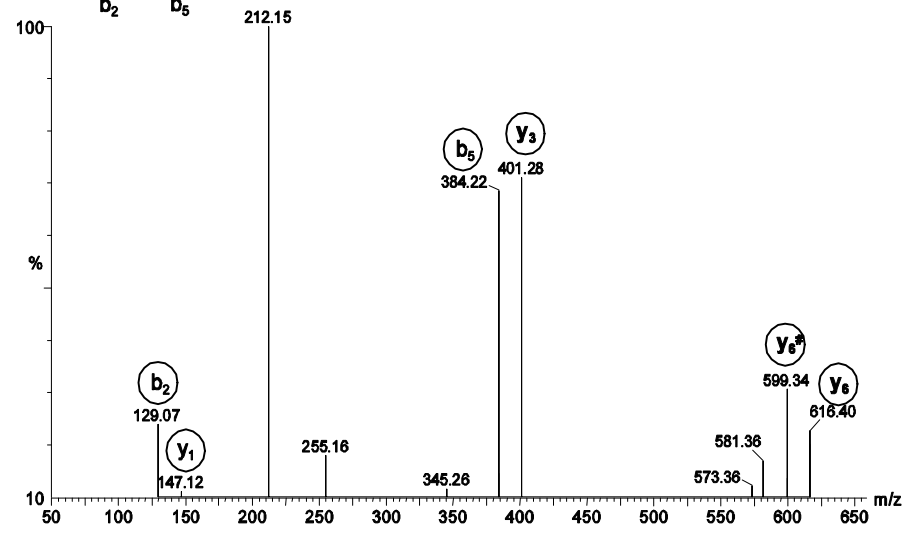

C

$y_{5} y_{4} y_{3} y_{1}$

$N]_{b_{2}}^{N} \int_{b_{4} b_{5}} R$

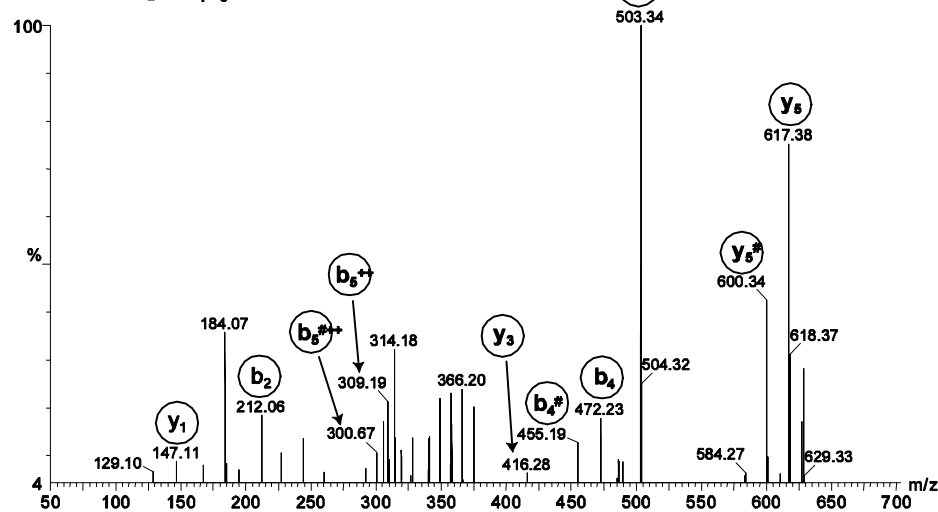




\section{Autoimmune IgG Preferentially Bind H1 Linker Histones in Activated Neutrophils}

and Neutrophil Extracellular Chromatin

To assess the range of SLE6-reactive proteins on Western blots, we prepared total cell lysates from unstimulated and Ca-ionophore stimulated human neutrophils. Lysates were resolved on SDS-PAGE, blotted onto nitrocellulose membranes and probed with SLE6 serum (Figure 3-4A). Antibodies bound numerous proteins from both $\mathrm{Ca}^{++}$ ionophore treated and untreated neutrophils, with notably little to no detectable binding to core histones. Binding of SLE6 to lysates showed that a protein in the molecular weight range of histone $\mathrm{H} 1$ along with few other proteins became more immunoreactive following treatment with $\mathrm{Ca}^{++}$ionophore (Figure 3-4A).

To examine the distribution of SLE6 antigen targets in cells, we stimulated neutrophils with LPS, an inflammatory stimulus that induces deimination and chromatin release from neutrophils (202), and used them for confocal microscopy. LPS-treated and untreated cells were incubated with SLE6, as well as a rabbit antibody to deiminated H3 and the DNA stain sytox orange (Figure 3-4B-D). SLE6 IgG binding increased in LPStreated neutrophils with decondensed chromatin (Figure 3-4C), whereas unstimulated neutrophils maintaining a lobed nuclear architecture showed minimal IgG binding (Figure 3-4B). In contrast, SLE6 IgG colocalized with deiminated H3 in decondensed intracellular chromatin (Figure 3-4C) and in some neutrophil NETs (Figure 3-4D). Intense granular SLE6 staining was observed in the cytoplasm of activated neutrophils, suggesting segregation of immunoreactive epitopes from the nuclear compartment before chromatin release (Figure 3-4D).

\section{Neutrophils Express Multiple H1 mRNAs and Proteins}

To obtain a more complete view of $\mathrm{H} 1$ subtype expression in neutrophils, we isolated total RNA from blood neutrophils and examined the representation of different H1 mRNA by hybridization to expression microarrays (Figure 3-5A). H1.4 was the most actively transcribed linker histone, followed by H1.0 and H1.2. In contrast, H1.1, H1.3 and H1.5 expression was near background levels (Figure 3-5A). The expression levels of the house-keeping gene, HGPRT were similar in magnitude to histone H1.2 (Figure 3-5A). These data indicate that mature neutrophils express a limited set of linker histones and that the bulk of newly made histones is comprised of H1.4.

Next, we asked whether the RNA present in mature neutrophils reflects the H1 protein complement that is present in neutrophils. For that purpose, we compared untreated human neutrophils to neutrophils stimulated with A23187 calcium ionophore for $2 \mathrm{~h}$. The $\mathrm{H} 1$ proteins were isolated from treated and untreated neutrophils by $5 \%$ perchloric acid extraction, separated by gel electrophoresis and probed with anti-citrulline antibodies (Figure 3-5B). H1 isolated from ionophore-treated cells reacted with the antibodies, indicating the presence of citrullines. In addition, the purified $\mathrm{H} 1$ resolved into a set of similar-sized proteins, suggesting the expression of different H1 subtypes. 


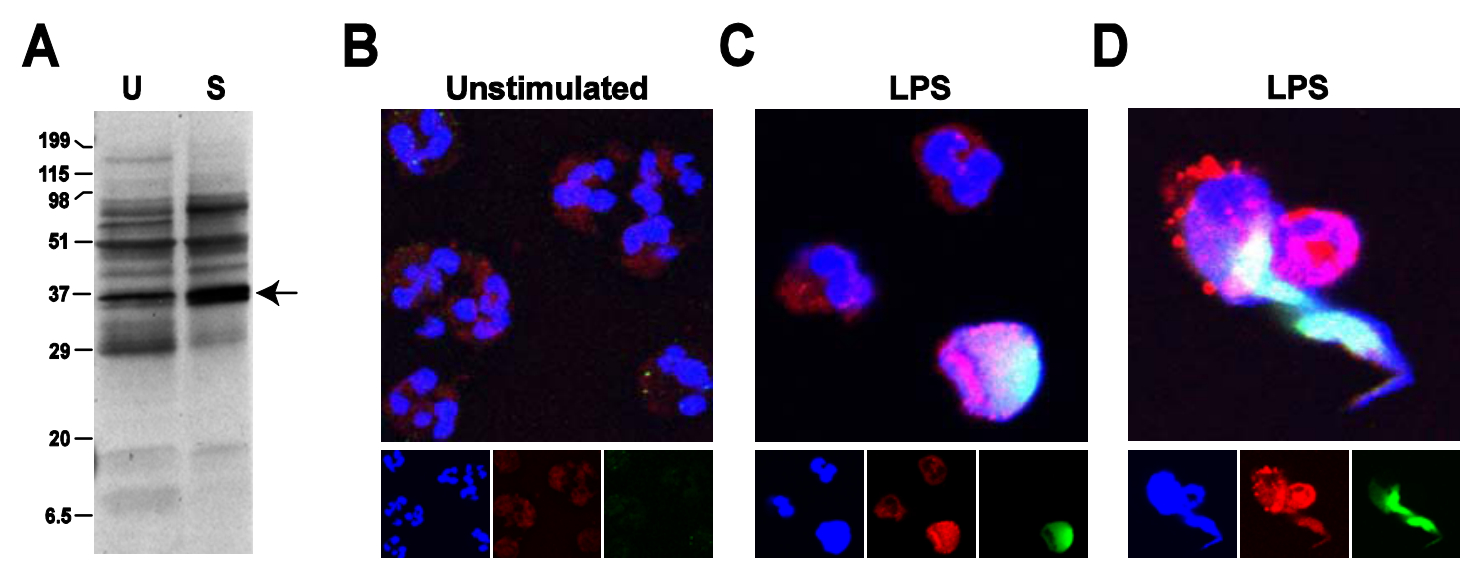

Figure 3-4: Neutrophil activation increases SLE6 reactivity

Lysates of unstimulated (U) and $\mathrm{Ca}^{++}$ionophore-treated (S) neutrophil were probed with SLE6 serum (A). Enhanced binding of IgG to a doublet of proteins migrating to a position near the $37 \mathrm{kD}$ marker is indicated (arrow). SLE binding (red) to unstimulated or LPS treated neutrophils was compared to the binding of anti-deiminated $\mathrm{H} 3$ antibody (green) and the DNA binding dye, sytox orange (blue) (B-D). Faint SLE6 binding to the cytoplasm of untreated neutrophils (B). SLE6 binding is increased in LPS-treated neutrophils that have lost the integrity of nuclear membrane $(\mathrm{C})$ and show increased reactivity with the anti-deiminated $\mathrm{H} 3$ antibody $(\mathrm{C})$. SLE6 bound to NETs that reacted with the anti-deiminated H3, although prominent cytoplasmic clusters of SLE6 binding were also observed (D). 
A B

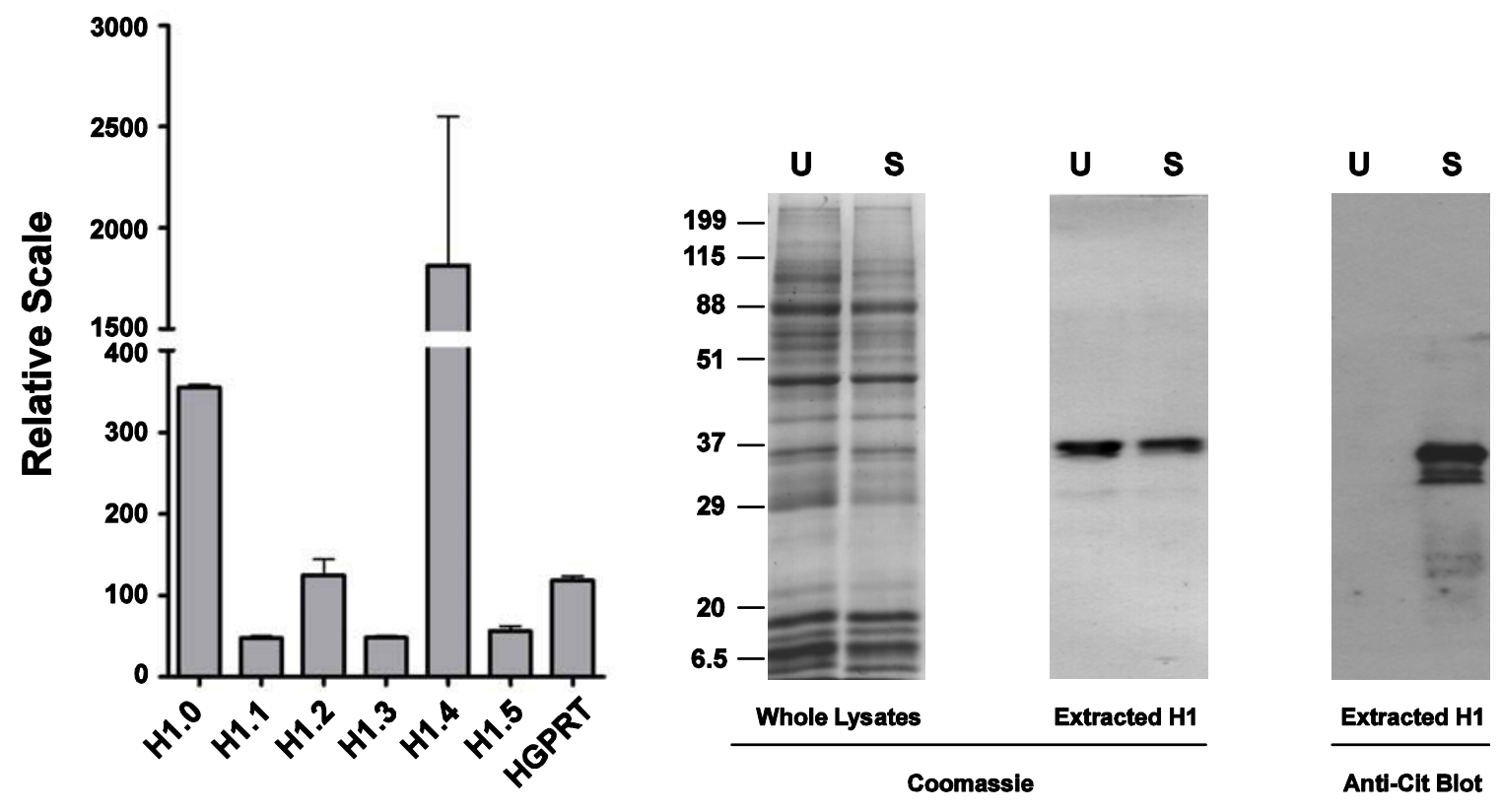

Figure 3-5: Analysis of linker histone transcripts and proteins from neutrophils Relative mRNA levels of different $\mathrm{H} 1$ subtypes were determined from total mRNA extracted from neutrophils. cDNA was prepared by reverse transcription and expression levels were measured on an Affymetrix Whole-Transcript Human Gene 1.0 ST Array. Relative fluorescence intensities were plotted for each H1 subtype (A). Whole cell lysates and perchloric-acid-extracted linker histones from unstimulated (U) and $\mathrm{Ca}^{++}$ ionophore-stimulated (S) neutrophils were analyzed by SDS-PAGE (coomassie). Extracted $\mathrm{H} 1$ histones were blotted, chemically modified and probed with anti-Cit (modified) antibody. Only H1 from activated neutrophils reacted with the antibody (B). 


\section{MS/MS Analysis of H1 Histones from Activated Neutrophils}

Following LysC digestion of $\mathrm{H} 1$ from activated neutrophils, mass spectroscopy identified the ER*SGVSLAALK peptide that may derive from H1.2, H1.3 or H1.4 (296). To confirm that the deimination of arginine to citrulline is consistent with the predicted peptide sequence, we performed MS/MS of the product-ions exclusively on the precursor ER*SGVSLAALK peptide (Figure 3-6A). The MS/MS spectrum contained a series of $\mathrm{y}$-ions $\left(\mathrm{y}_{1}-\mathrm{y}_{9}\right)$ that did not contain $\mathrm{R}^{*}$. However, the $\mathrm{b}$-series ions $\left(\mathrm{b}_{2}-\mathrm{b}_{10}\right)$ exhibited masses consistent with citrulline: the $\mathrm{b}_{2}{ }^{\#}\left(\mathrm{~b}_{2}-\mathrm{NH}_{3}\right), \mathrm{b}_{4}{ }^{@}\left(\mathrm{~b}_{4}-\mathrm{H}_{2} \mathrm{O}\right)$, and $\mathrm{b}_{10}$ ion were found at $\mathrm{m} / \mathrm{z} 270.11,413.21$, and 985.60 , respectively. The mass errors were $0.02,0.03$, and $0.07 \mathrm{Da}$, respectively for an average error of $0.04 \mathrm{Da}$. Therefore, the masses of all the bions closely matched the masses that are predicted by the presence of citrulline instead of arginine at position 53 of H1.2 (Figure 3-3A). Similar arguments apply to the homologous peptide that was derived from histone H1.5 (ER*NGLSLAALK, Figure 3-6C), in that masses corresponding to the $b_{3}, b_{7}, b_{8}, b_{9}$ and $b_{10}$ ions closely matched those that are predicted if citrulline replaces arginine.

Interestingly, the $\mathrm{H} 1$ histones that were purified from activated neutrophils also yielded peptides that were identical to the ones described above except for the presence of arginines (Figure 3-6B and D). There, the $b$ and $y$ series ion masses were consistent with the presence of arginine, indicating that a $2 \mathrm{~h}$ treatment of neutrophils with ionophore does not completely convert histone $\mathrm{H} 1$ arginines to citrullines. Moreover, we observed the deimination of arginines in unique peptides derived from histone H1.0: GVGASGFR*LAK (Figure 3-6E) and R*LVTGVLK (Table 3-1). In contrast, we found no evidence for deimination of the arginine in NNSRIK that is part of the third helix in the globular domain (Figure 3-6F). Our results suggest that several arginines in the globular DNA binding domain of $\mathrm{H} 1$, including R53, the most conserved arginine in $\mathrm{H} 1$ histones, are converted to citrulline following neutrophil activation (Table 3-1).

\section{Summary of Histone H1 Expression in Neutrophils}

The mass spectrometry experiments provided evidence supporting the expression of at least 5 different $\mathrm{H} 1$ proteins in neutrophils. We identified peptides whose sequences unambiguously matched unique sequences in each of 5 different $\mathrm{H} 1$ subtypes (H1.0, H1.2, H1.3, H1.4 and H1.5; see Figure 3-7). It is interesting that we detected a peptide matching H1.3 (VAGAATPK) and two peptides matching H1.5 (KPAGATPK and KPAAAGVK), despite very low mRNA for H1.3 and H1.5 (Figure 3-5A). Overall, protease cleavage and mass spectrometry yielded a sequence coverage of $30-44 \%$ for each of the $\mathrm{H} 1$ proteins, although some peptides were shared between the most closely related H1 subtypes (Figure 3-7). 
Figure 3-6: $\quad$ MS/MS analysis of $\mathrm{H1}$ peptides from activated neutrophils $\mathrm{MS} / \mathrm{MS}$ spectra of peptides yielding doubly charged precursor ions of $\mathrm{H} 1$ histone from $\mathrm{Ca}^{++}$ionophore treated neutrophils (A-F). Fragmented ions (b series and y series) are marked on the peptide sequences or spectral peaks. Both peptide ER*SGVSLAALK (A) and ERSGVSLAALK (B) were observed, with a +1Da shift resulting from deimination of arginine in evidence in $b_{2}$ and $b_{10}$ ions. The peptide ERSGVSLAALK is conserved between H1.2, H1.3 and H1.4 subtypes. Both ER*NGLSLAALK (C) and its non-deiminated form ERNGLSLAALK (D) derived from H1.5 were identified. Again, the mass shift of corresponding $b$ ions is consisten with arginine deimination. Argininecontaining peptide GVGASGFR*LAK (E) from H1.0 was also identified, whereas peptide NNSRIK (F), conserved in subtypes H1.1-H1.5 was only found in its non-deiminated form. 
A

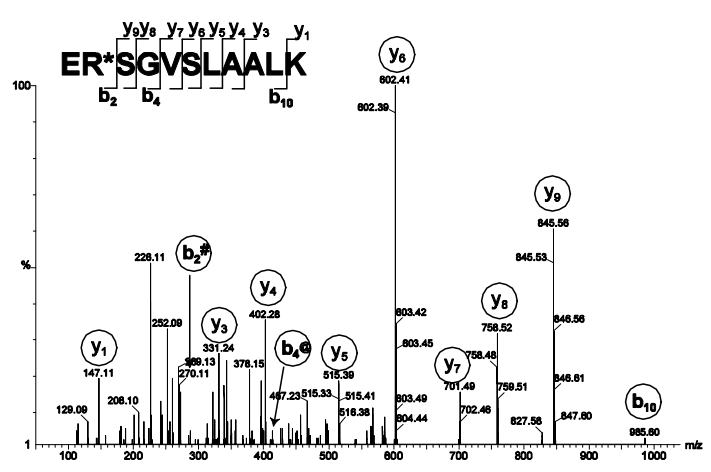

C

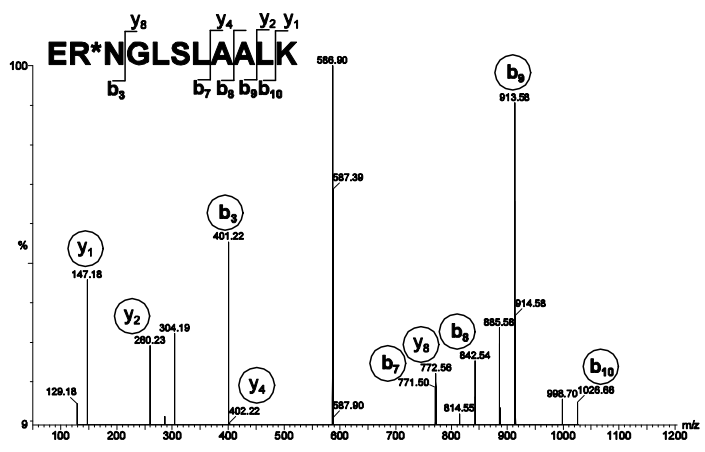

E



B

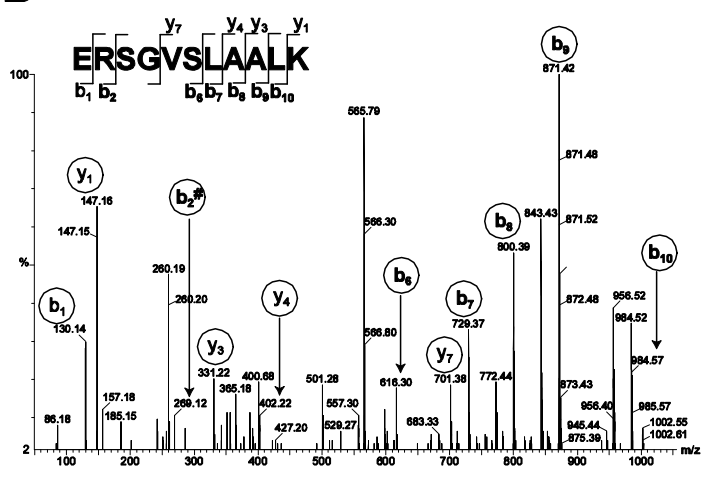

D

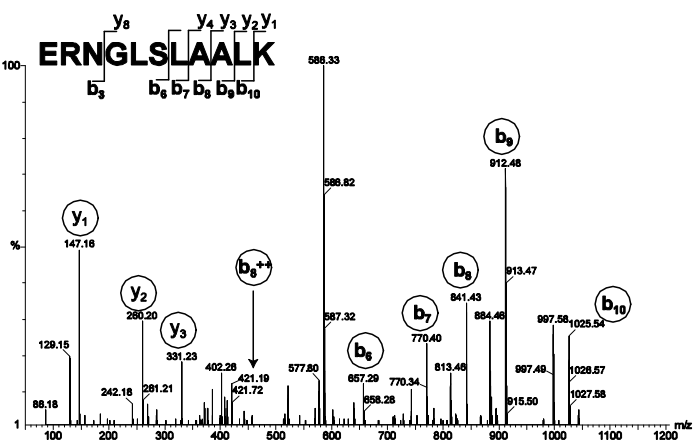

F

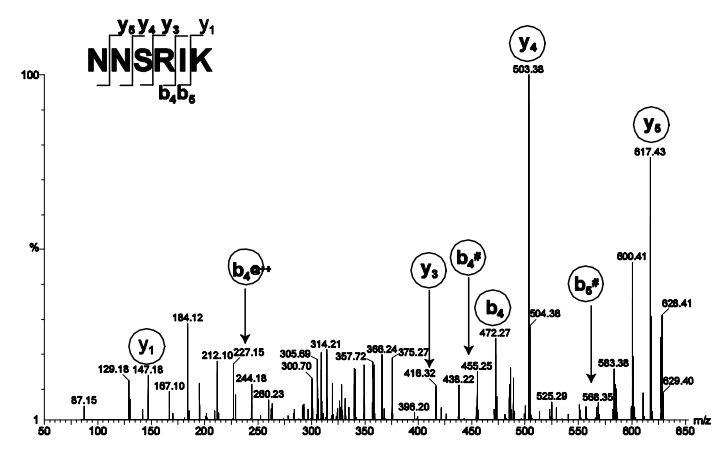


Table 3-1: Arginine containing peptides detected in $\mathrm{H1}$ extracted from activated neutrophils

\begin{tabular}{ccc}
\hline Deiminated Peptides & Non-modified Peptides & Corresponding H1 Isotype \\
\hline ER*SGVSLAALK & ERSGVSLAALK & $\mathrm{H} 1.2-\mathrm{H} 1.4$ \\
ER*NGLSLAALK & ERNGLSLAALK & $\mathrm{H} 1.5$ \\
GVGASGFR*LAK & - & $\mathrm{H} 1.0$ \\
R*LVTTGVLK & - & $\mathrm{H} 1.0$ \\
- & NNSRIK & $\mathrm{H} 1.1-\mathrm{H} 1.5$ \\
\hline
\end{tabular}

* Indicate deimination of arginine to citrulline. 


\begin{tabular}{|c|c|c|}
\hline 2 & . & AAPAAAPPAEKAPVKKKAAKKAG-GTP--RKASGPPVSELITKAVAASKERSGV \\
\hline 1.3 & 1 & SETAPLAPTIPAPAEKTPVKKK-AKKAG-ATAGKRKASGPPVSELITKAVAASKERSGVSLAALKKALAAAGYDVEKNNSRIKLGLKSLVSKGTLVQTKGTGASGSFK \\
\hline 1.4 & 1 & SETAPAAPAAPAPAEKTPVKKKARKS---AGAAKRKASGPPVSELITKAVAASKERSGVSLAALKKALAAAGYDVEKNNSRIKLGLKSLVSKGTLVQTKGTGASGSFKLNKKAA \\
\hline 1.5 & 1 & KERNGLSLAALKKALAAGGYDVEKNNSRIKLGLKSLVSKGTLVQTKGTGASGSFKLNKKAA \\
\hline 1.1 & 1 & ALKKALAAAGYDVEKNNSRIKLGIKSLVSKGTLVQTKGTGASGSFKLNKKAS \\
\hline 1.0 & 1 & TENSTSAPAAK------PKRAKASKKST--------DHPKYSSDMIVAAIQAEKNRAGSSR \\
\hline & & ----- \\
\hline 1.3 & 103 & AAKPRKPAGAAKKPKKVAGAATPKKSIKKTPKKVKKPA \\
\hline 1.4 & 102 & -АKK \\
\hline 1.5 & 105 & SGEAKPKAKKAGAAKAKKPAGAT--PKKAKKAAGAKKAVKKTPKKAKKPAA \\
\hline . & 5 & ETKPGASKV--ATKTKATGASKKLKKATGAS--KKSVK \\
\hline L. 0 & & KSVAFKKTKKEIKKVATPKKASKPKKAASKAPTKKPKATPV \\
\hline
\end{tabular}

Figure 3-7: Summary of mass spectrometry with linker histones from activated neutrophils

Sequences of somatic linker histone H1 subtypes were aligned to maximize homology. Arginine residues are indicated in red. Peptides identified by mass spectrometry highlighted in yellow, and H1 subtype-specific peptide sequences are in black and underlined. The globular domain of H1 is indicated by a broken line above the sequences. Brown boxes represent the 3 helices of the H1 winged helix. Residues contributing to DNA binding residues are indicated with arrows. 


\section{Discussion}

Evidence mounts that NETs participate in the induction of autoimmune responses. Because NETs are released at sites of inflammation (306-309) and NET chromatin intimately associates with bacteria $(194,310)$, NETs were predicted to contribute to autoimmunity (285). Recent observations support this prediction. Autoantibodies from patients with systemic autoimmunity recognize innate immune defense proteins that associate with NETs. This is the case for the major NET components, including the granule proteases elastase, cathepsin $\mathrm{G}$ and proteinase $3(235,236,238)$, as well as for various bactericidal peptides that are stored in granules and co-purify with NETs, such as neutrophil defensins and the LL37 peptide $(239,240)$. Therefore it is relevant to further characterize mechanisms involved in the release of NET chromatin.

NET release depends on PAD4 $(213,302)$. Although PAD4 deiminates a variety of substrates $(283,303)$, it is plausible that histone deimination plays an important role in the unfolding of nuclear chromatin into the extended structure of NETs. Consistent with this role, treatment of a nucleosome array with PAD4 decreased its capacity for compaction in the presence of linker histone H5 (311). Our studies have revealed that NETs contain deiminated histones (202). Deiminated histones thus can be considered as markers of NET chromatin $(312,313)$.

In that light, our recent studies support the idea that NETs induce autoantibody responses in susceptible individuals. We screened sera from patients with SLE, RA and Felty's syndrome and found examples of sera that preferentially bound to deiminated histones (303).

Our current results confirm that human autoimmune IgG react with the deiminated form of human H1.2. Autoantibodies to deiminated histone H1 provide support for the hypothesis that NETs induce autoantibodies. However, only a small fraction of the tested sera $(5 \%)$ had antibodies with a marked preference for the citrullinated form of $\mathrm{H} 1$. Antibodies to linker histones $\mathrm{H} 1$ are considered as important marker SLE as dsDNA. Detection of antibodies against H1 by ELISA yield a sensitivity of about $45 \%$ with a specificity of $98 \%$ in diagnosing SLE (314). H1 antibodies show a strong association with disease activity such as lupus nephritis (314) and neuropsychiatric lupus (315). H1 is also an important autoimmune target in Sjögren's syndrome as binding of purified IgG against an immunodominant epitope of La/SSB antigen was completely inhibited by $\mathrm{H} 1$ (316). Involvement of $\mathrm{H} 1$ in lupus is not limited to being an autoimmune target. PBMCs co-cultured with $\mathrm{H} 1$ from lupus patients, but not healthy controls, induced increased production of TNF-alpha (317). Whether antibodies to deiminated $\mathrm{H} 1$ derive from a subset of patients with particular clinical features is unknown.

Our results identify the complement of $\mathrm{H} 1$ subtypes expressed in human neutrophils. Although the H1.4 gene encodes the majority of mRNA in mature neutrophils (Figure 3-5A), neutrophils contain at least five H1 subtypes (Figure 3-7), including H1.3 and H1.5 for which very little if any mRNA could be detected. This 
observation suggests that $\mathrm{H} 1.3$ and $\mathrm{H} 1.5$ may be synthesized during neutrophil development but that they are replaced with H1.0, H1.2 and particularly with H1.4 as neutrophils mature. Because $\mathrm{H} 1$ histones play important regulatory roles in chromatin compaction (296), it is likely that changes in H1 subtype expression reflect changes in chromatin conformation, condensation, or nuclear morphology. A shift in subtype expression may alter global chromatin properties because different $\mathrm{H} 1$ subtypes differ in their DNA binding and/or protein interaction potential.

The binding of SLE6 serum IgG to an epitope containing citrulline at position 53 of the $\mathrm{H} 1$ winged helix was surprising because the arginine at position 53 was considered to be a DNA binding residue (318). DNA bound to arginine 53 may shield this domain of histone $\mathrm{H} 1$ from antibody binding. Moreover, arginine 53 is conserved in all human $\mathrm{H} 1$ subtypes and is located within the structurally conserved globular region of $\mathrm{H} 1$ (Figure 3-7). However, the involvement of R53 in DNA binding was deduced from mutagenesis of this arginine in histone H1.0 (318). A recent study which examined this residue in $\mathrm{H} 1.2$ found only a negligible effect of an alanine substitution on DNA binding (319). The authors suggested that the 53 position is not part of the DNA binding site and that their result may explain why H1.0 binds more tightly to DNA than H1.2 (319). This result may imply that H1.2 has only two DNA binding sites, whereas H1.0 has three.

If that conclusion holds true, then R53 must have another function that is highly conserved in evolution. It could be that R53 contributes to one or more of the protein interactions that have been identified for $\mathrm{H} 1$ histones. These range from interactions with core histones in chromatin (320) to interactions with RNA polymerase (299) and various transcription factors $(321,322)$. Because the two main $\mathrm{H} 1$ subtypes that are expressed in mature neutrophils, H1.2 and H1.4, may not use R53 for DNA binding, it may be that this residue has an important role in the specific nuclear chromatin changes that take place in neutrophil activation. 


\title{
CHAPTER 4. DEIMINATION REDUCES REACTIVITY OF HISTONES WITH MURINE LUPUS AUTOANTIBODIES
}

\begin{abstract}
Summary
Deimination of histones, a posttranslational modification of arginine to citrulline by peptidylarginine deiminase 4 (PAD4), is associated with neutrophil extracellular traps (NETs) and may compromise tolerance of self-antigens. The aim of this study was to test whether spontaneously autoimmune mice react preferentially with deiminated or nondeiminated histones.

IgG $\mathrm{Ab}$ binding to deiminated histones and non-deiminated histones were determined by ELISA and Western blotting, and splenocyte proliferation was analyzed in spontaneously autoimmune mice. We measured levels of deiminated histones in tissues from C57BL/6.Sle1.Sle2.Sle3 (B6.TC) and control mice, to search for putative tolerogens. Serum IgG from autoimmune (NZBxNZW)F $1, N_{2}$ 2410, and C57BL/6.Sle1.Sle3 mice consistently bound non-deiminated histones with preference

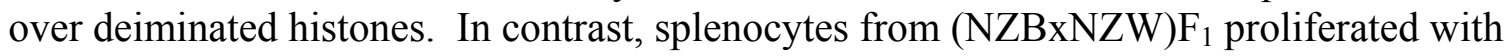
no bias for either antigen. In addition, the bone marrows and spleens of B6.TC mice contained increased amounts of deiminated histones as compared to control C57BL/6 mice.

Our study demonstrates that sera of autoimmune lupus prone mice react less strongly against deiminated histones than non-deiminated histones. We note that deiminated histones, presumably derived from NETs, are available at increased levels in primary and secondary lymphoid tissues of autoimmune mice relative to littermates. The deiminated histones may function as tolerogens to suppress production of anti-deiminated histone $\mathrm{IgG}$.
\end{abstract}

\section{Introduction}

Connections between the innate and adaptive immune system are intriguing and often surprising. This is particularly true with regard to mechanisms that distinguish self from non-self (323). A special challenge for adaptive immunity arises during neutrophil responses to infections. Neutrophils respond to bacterial infections by ejecting nuclear chromatin from the confines of the cell (194). The chromatin is associated with various bactericidal substances and it forms a mesh that immobilizes infectious organisms (324). These structures are called neutrophil extracellular traps (NETs). NETs serve to capture bacteria and fungi. It is important to efficiently clear NETs, as they can promote intravascular thrombosis (307).

The challenge for the adaptive immune response is to use the NET-ted bacteria as antigens, while maintaining tolerance to the structural components of NETs. A possible confounding factor in the recognition of NETs may be that their release is accompanied 
by a particular post-translational modification (PTM). Neutrophils exposed to inflammatory stimuli, or the physical proximity of microbes, induce the enzyme peptidylarginine deiminase 4 (PAD4) which converts arginines to citrullines (202).

Deimination of histones appears to play a special role in the release of NETs, as mice deficient in PAD4, the enzyme that deiminates histones, fail to make NETs and suffer more serious infections (213). Deimination may have broader physiological roles and yet several deiminated self-proteins are targeted by autoantibodies. Deimination of keratin and filaggrin occur during terminal differentiation of keratinocytes $(209,210$, 325 ) yet deiminated filaggrin is a target of antibodies (Abs) associated with rheumatoid arthritis (RA) (214). Generally, anti-citrullinated protein antibodies (ACPA) serve as reliable diagnostic markers for RA (326). Deimination of vimentin, a member of the intermediate filament family of cytoskeletal proteins, generates the target of anti-Sa autoantibodies in RA (264). It is not clear whether normal levels of deimination generate autoepitopes in RA or whether the deimination in RA is enhanced and produces unusual "neo" epitopes.

PAD4 is the only PAD that has a nuclear localization signal and deiminates histones H2A, H3, and H4 $(268,327)$. In a genetic study, a link between PAD4 and RA was observed: Haplotypes linked to increased RA susceptibility produced PAD4 mRNA that was more stable than mRNA from non-susceptible haplotypes (228). The increased mRNA stability may lead to increased expression of PAD4 in individual carriers of these susceptibility haplotypes. The association between deiminated proteins and various autoimmune diseases strongly suggests that deimination generates novel epitopes that are able to break tolerance and initiate autoimmune responses. However, it is not known whether deimination of histones is sufficient to break tolerance to nuclear autoantigens.

Recently, it has been suggested that NETs are responsible for inducing autoimmune reactions $(242,328,329)$. Type 1 IFN, involved in lupus pathogenesis, has been found to prime neutrophils to release NETs (328). Consequences of NET release are exposure of DNA-peptide complexes (242) that cause further release of Type 1 IFN from plasmacytoid dendritic cells (329). Also, the structural components of NETs and various associated proteins and peptides have been recognized as autoantigens in lupus patients $(260,330,331)$. Based on these facts and our observation that chromatin released in NETs consist of deiminated histones, we investigated the hypothesis that if NETs are induced by inflammation, as is typical of autoimmunity, and if NETs are immunogenic, then anti-deiminated histone antibodies should be prevalent in lupus-prone mice.

IgGs from $(\mathrm{NZBxNZW}) \mathrm{F}_{1}$ mice and their recombinant derivative strains, including NZM2410 and B6.Sle1, B6.Sle1.Sle3 or B6.TC congenics, showed strong preference for non-deiminated histones over deiminated histones by ELISA and Western blot. Thus, even after tolerance to histones was broken and autoantibodies to nondeiminated histones were expressed, autoimmune-prone congenic strains remained tolerant of deiminated histones. B cell binding to deiminated histones relative to nondeiminated histones was reduced. Therefore, autoantibody binding focused on PAD4 
substrate arginines. No such bias was observed with autoreactive splenocytes from autoimmune $(\mathrm{NZBxNZW}) \mathrm{F}_{1}$ mice. These splenocytes responded differentially to deiminated histones versus non-deiminated histones and the responses developed simultaneously with serum anti-DNA autoantibodies. The splenocyte response was idiosyncratic in preferentially targeting either deiminated histones or non-deiminated histones. The $\mathrm{B}$ cell bias against deiminated histones argues that deiminated histones remain effective tolerogens in autoimmune mice. In support of this possibility, we observed elevated levels of deiminated histones in bone marrows and spleens of autoimmune B6.TC mice. Our observations suggest that, even in overtly autoimmune lupus mice, tolerance mechanisms inhibit B cells that react with a deiminated variant of an important nuclear autoantigen.

\section{Methods}

\section{In-vitro Histone Deimination}

Calf thymus histones (US Biochemicals) were dissolved in PAD4 reaction buffer (100 mM Tris- $\mathrm{HCl}$ at $\mathrm{pH} 7.6,50 \mathrm{mM} \mathrm{NaCl}, 2 \mathrm{mM}$ DTT and $10 \mathrm{mM} \mathrm{CaCl}_{2}$ ) to a final concentration of $0.1 \mathrm{mM}$ total histones. The reaction mixture was incubated for $10 \mathrm{~min}$ at $37^{\circ} \mathrm{C}$ and then PAD4 enzyme was added to a final concentration of $0.2 \mu \mathrm{M}$ to start the reaction. Sixty $\mu 1$ aliquots were taken at different time points and the reaction was quenched with liquid nitrogen. Amounts of deiminated proteins were estimated by colorimetry as described (332) using L-citrulline (Sigma) as a standard. Deimination was also confirmed by Western blot using anti-deiminated histone antibody from Abcam (Cat \# ab5103) that recognizes citrullines at positions 2, 8, and 17 of histone $\mathrm{H} 3$.

\section{Immunization of Mice}

Experiments were performed on 4-6 week female BALB/c mice housed in the animal care facility of the University of Tennessee Health Science Center, Memphis USA. All experimental protocols were reviewed and approved by the Institutional Animal Care and Use Committee (IACUC). BALB/c mice were divided into different groups for immunization. Four mice each were immunized intraperitoneally with $100 \mu \mathrm{g}$ of non-deiminated histones or deiminated histones. Two mice were immunized with 100 $\mu \mathrm{g}$ of ovalbumin (OVA) as a positive control. Initial immunizations were done with emulsified proteins in complete Freund's adjuvant (CFA) and one subsequent immunization was done in incomplete Freund's adjuvant (IFA). Mice were boosted 10 days after the first immunization, and sera were collected 7 days later and used for comparison in ELISA. In addition, two mice each from the deiminated histone and nondeiminated histone groups were boosted with $100 \mu \mathrm{g}$ of deiminated histone or nondeiminated histone and $20 \mu \mathrm{g}$ of Imiquimod (Invivogen), a TLR-7 stimulant, along with IFA. The remaining two mice from each group were boosted with $100 \mu \mathrm{g}$ of deiminated histones or non-deiminated histones in IFA. One mouse from the OVA group was also 
boosted with $100 \mu \mathrm{g}$ of OVA along with $20 \mu \mathrm{g}$ of Imiquimod in IFA. Sera were analyzed seven days after each immunization.

\section{ELISA}

Sera were obtained from (NZBxNZW)F 1 , NZM2410, B6.Sle1, B6.Sle1.Sle3 and B6.TC mice at 7 months of age when these mice produce autoantibodies. The treatment and care of animals were in accordance with the guidelines of the IACUC of UTHSC, the University of Florida and the Norwegian Ethical and Welfare Board. Deiminated histones for ELISA and Western blot were prepared in vitro. Deimination of nondeiminated histones (Calf thymus histones, US Biochemicals) was done by recombinant PAD4 as described above. Flat bottom, 96 well microtiter plates (Immulon 4HBX; Thermo Electron Corp.) were coated overnight with $5 \mu \mathrm{g} / \mathrm{mL}$ of deiminated histones, non-deiminated histones, OVA (Sigma) or coating buffer alone (control). Plates were washed 3 times with $0.05 \%$ Tween-20 in PBS and blocked with $2.5 \%$ BSA in $0.02 \%$ $\mathrm{NaN}_{3}$ and PBS for $2 \mathrm{~h}$. A 1:200 initial dilution of primary sera along with 5-fold serial dilutions in 1.6\% Tween-20 and 1\% BSA in PBS were incubated for $2 \mathrm{~h}$ in plates. Then, serum dilutions were removed and wells were washed with $0.1 \%$ Tween-20 in PBS. Alkaline phosphate-conjugated goat anti-mouse IgG ( $\gamma$-chain-specific; Southern Biotech) was added at 1:1000 dilution in 1\% BSA with 0.05\% Tween-20 in PBS for $1 \mathrm{~h}$.

Phosphatase substrate (Sigma) was used to develop the ELISA and OD values were read at $405 \mathrm{~nm}$ on a Multiskan Plus plate reader (Labsystems).

ELISA with the sera from immunized BALB/c mice was done with the same protocol as described in methods with a modification such that the initial serum dilutions tested were 1:100 with 1:3 serial dilutions, whereas as initial serum dilutions for autoimmune mice were 1:200 with 1:5 serial dilutions.

Serum antibodies against dsDNA were detected by ELISA exactly as described $(333,334)$. In short, calf-thymus dsDNA (10ug/ml in PBS) was coated on microtitre plates (MaxiSorb; Nunc, Copenhagen, Denmark). Sera from mice were diluted 2 fold from 1:100 to 1:3,200 in PBS containing 0.02\% Tween-20 and incubated in wells. ELISA readings were obtained with peroxidase-conjugated rabbit anti-mouse Fc- $\gamma$ antibodies.

\section{Western Blot}

For Western blot analysis, proteins were resolved on 15\% SDS-PAGE and transferred to nitrocellulose. Membranes were blocked in 5\% BSA in $0.1 \%$ Tween 20 in TBS (TBST) overnight at $4^{\circ} \mathrm{C}$. Subsequently, the membranes were incubated with sera at 1:500 dilution in TBS containing 2.5\% BSA, 0.5\% NP-40 and 0.05\% SDS. After $2 \mathrm{~h}$ of incubation, membranes were washed with $1 \%$ NP-40 in TBS. Anti-mouse IgG-HRP (H+L-chain-specific; Millipore) was used for detection at 1:10,000 dilution in TBST for $1 \mathrm{~h}$ and blots were developed using "Western Lightning" chemiluminescence 
(PerkinElmer). Separately, autoimmune sera were treated with DNase1 prior to Western blotting. Briefly, 400ul of a 1:200 dilution of sera were incubated with 20 units of DNase1 (New England Biolabs) for $1 \mathrm{~h}$ at room temperature before adjusting the sera to a 1:500 dilution in Western blot binding buffer. To probe for deiminated histone $\mathrm{H} 3$ in B6.TC and C57BL/6 mice, equal amount of tissue lysates were resolved on $12 \%$ SDS-PAGE and transferred to nitrocellulose. Membranes were blocked with 5\% BSA in TBST for 30 min and incubated with anti-deiminated histone $\mathrm{H} 3$ rabbit antibody (Abcam, ab 5103) overnight at $4^{\circ} \mathrm{C}$. Membranes were washed and incubated with HRPconjugated goat-anti-rabbit secondary antibody for $1 \mathrm{~h}$ at room temperature, washed three times in TBST and twice in TBS alone. The HRP activity was detected as above.

\section{Splenocyte Proliferation Assay}

$\mathrm{BALB} / \mathrm{c}$ mice were boosted twice with $100 \mu \mathrm{g}$ antigen (deiminated histones, non-deiminated histones or OVA) 14 and 2 days prior to the splenocyte proliferation assay. Ninety-six well tissue culture plates (Corning Incorporated) were filled with 100 $\mu \mathrm{l}$ aliquots of $100 \mu \mathrm{g} / \mathrm{ml}$ (or 3 fold serial dilutions) of deiminated histones, nondeiminated histones, or ovalbumin in RPMI 1640 (Mediatech Inc.) supplemented with $10 \%$ FBS. Splenocytes were isolated and resuspended in RPMI with $10 \%$ FBS at $1 \times 10^{6}$ cells $/ \mathrm{ml}$. One hundred $\mu 1$ of cell suspension was added to each well and plates were incubated at $37^{\circ} \mathrm{C}$ in $5 \% \mathrm{CO}_{2}$ for 72 to $96 \mathrm{~h}$. Tritiated thymidine $(1 \mu \mathrm{Ci} /$ well $)$ was added for the last $17 \mathrm{~h}$ of incubation. Plates were harvested onto glass fiber filters and thymidine incorporation was assessed by scintillation counting.

Splenocyte proliferation assays for autoimmune mice used splenocytes from female (NZBxNZW) $F_{1}$ mice of different ages purchased from the Jackson Laboratory (Bar Harbor, ME). Following RBC lysis, splenocytes were resuspended in DMEM-10 media with $10000 \mathrm{U} / \mathrm{ml}$ penicillin and $10 \mathrm{mg} / \mathrm{ml}$ streptomycin. One hundred thousand cells were incubated with deiminated histones or non-deiminated histones $(20 \mathrm{ug} / \mathrm{ml}$ of protein $)$ in triplicate wells. Tritiated thymidine incorporation $(1 \mu \mathrm{Ci} /$ well $)$ was measured after 20 h, 3 days or 6 days by liquid scintillation, as described $(335,336)$.

\section{Ex-vivo Tissue Lysate Preparation}

Seven-month-old B6.TC female mice and age-matched control C57BL/6 IgHa were dissected. A portion of spleen, bone marrow, kidney and liver was cut, minced with scissors and crushed between two sterile frosted glass slides. Dissociated tissues were washed in PBS (without $\mathrm{Ca}^{++}$) and centrifuged at $5000 \mathrm{~g}$ for 5 min to pellet cells. Cell pellets were mixed with lysis buffer (65mM Tris $\mathrm{pH} 7.2$, 2\%SDS, 10\% Glycerol), containing protease inhibitors. To test for deiminated histones and total $\mathrm{H} 3$ in tissue lysates, equal amounts of total protein were analyzed by Western blotting, as described above. 


\section{Results}

\section{In-vitro Histone Deimination}

For the immunization and immunochemistry experiments, we prepared calf thymus deiminated histones by using recombinant PAD4 (274). The deimination reaction reached completion by $24 \mathrm{~h}$ (Figure 4-1A), as addition of more PAD4 enzyme to the reaction at that time did not increase deimination (data not shown). We measured the generation of 1.67 moles of citrulline per mole of total histones. Because histone $\mathrm{H} 2 \mathrm{~B}$ is not a substrate for PAD4, we estimate that about 2 citrullines were introduced into each modifiable core histone. Quantitation of citrullines was performed by colorimetry (Figure 4-1A). We confirmed histone deimination by Western blot using antibodies that recognize citrullines at the histone $\mathrm{H} 3$ amino terminus (Figure 4-1B). The starting calf thymus histone preparation had no detectable citrullines (Figure 4-1B, time $=0$ ).

\section{Spontaneously Arising Anti-histone Autoantibodies Bind Non-deiminated Histones}

To assess the production of anti-deiminated histones autoantibodies in mice that spontaneously develop anti-nuclear autoantibodies (ANA), we tested sera from $(\mathrm{NZBxNZW}) \mathrm{F}_{1}$ and their recombinant inbred derivative strain NZM2410 for binding to non-deiminated histones and deiminated histones. In addition, we tested the contribution of lupus-predisposing genetic intervals Sle1 and Sle3 that were back-crossed from the NZM2410 onto the C57BL/6 background (B6.Sle1 and B6.Sle1.Sle3). Sle1 is a locus that breaks tolerance to chromatin, whereas Sle3 affects functions of myeloid cells (53). We also tested B6.TC mice, a triple congenic strain that in addition to having the lupuspredisposing genetic intervals Sle1 and Sle3, also carries the Sle2 interval that results in the spontaneous activation and hyperactivity of B cells. The parental strains, NZB and $\mathrm{NZW}$, have distinct $\mathrm{MHC}, \mathrm{H}-2^{\mathrm{d}}$ and $\mathrm{H}-2^{\mathrm{z}}$ respectively. The lupus-predisposing $\mathrm{H}-2^{\mathrm{Z}}$ was maintained in the NZM2410 mice, whereas the congenics have the $\mathrm{H}-2^{\mathrm{b}}$ from C57BL/6. Antibody binding to deiminated histone and non-deiminated histone was assessed by ELISA (Figure 4-2) and Western blot (Figure 4-3 and Table 4-1). As controls, sera from age- and sex-matched C57BL/6 mice were used.

By ELISA, (NZBxNZW)F 1 (Figure 4-2A), NZM2410 (Figure 4-2B), B6.Sle1 (Figure 4-2C), B6.Sle1.Sle3 (Figure 4-2D) and B6.TC (Figure 4-2E) sera showed preference for non-deiminated histones over deiminated histones. This preferential

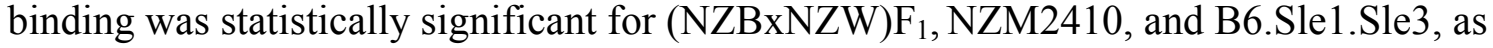
assessed by a paired, two tailed T-test. The strength of binding differed for different mice and serum dilutions but binding to non-deiminated histones was equal or greater than the binding to deiminated histones in all except one B6.TC mouse. We also confirmed the additive effect of Sle1 and Sle3 loci, as the B6.Sle1.Sle3 combination resulted in greater absorbance values relative to the B6.Sle1 mice. In parallel assays, sera from C57BL/6 mice (Figure 4-2F) showed no reactivity to histones. Both deiminated histones and non-deiminated histones were present in equivalent concentrations on the 

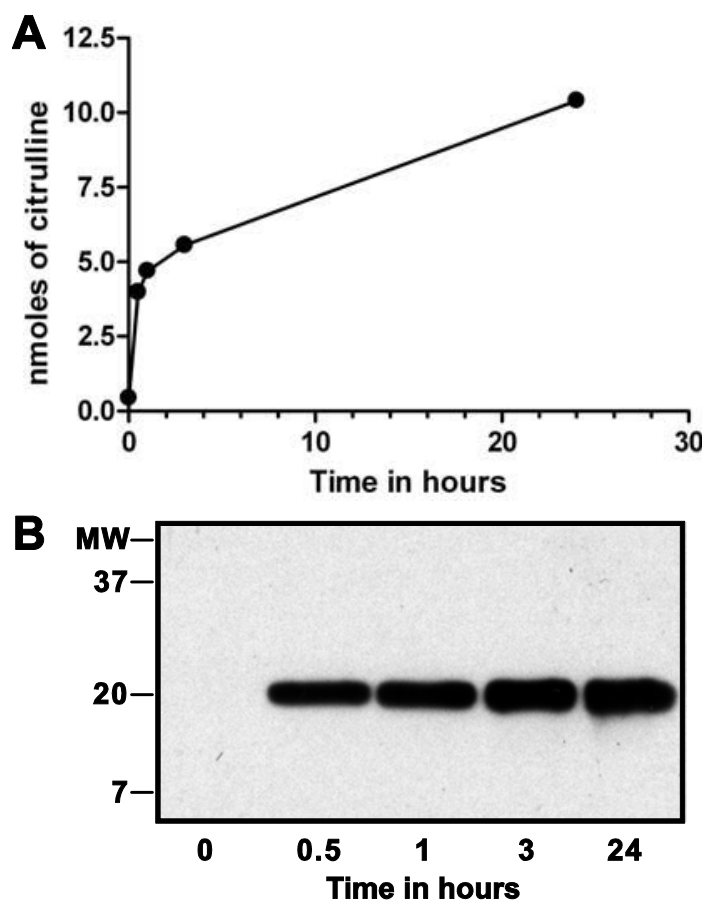

Figure 4-1: In-vitro deimination of histones by PAD4

Calf thymus histones $(0.1 \mathrm{mM})$ were incubated with $0.2 \mu \mathrm{M}$ PAD4 up to $24 \mathrm{~h}$ and deimination (in nmoles) was measured by colorimetry at $595 \mathrm{~nm}$ using a citrulline standard solution. B) Western blot showing deimination of calf thymus histones at different time points in a $24 \mathrm{~h}$ in-vitro PAD4 reaction using anti-deiminated histone $\mathrm{H} 3$ antibody. 

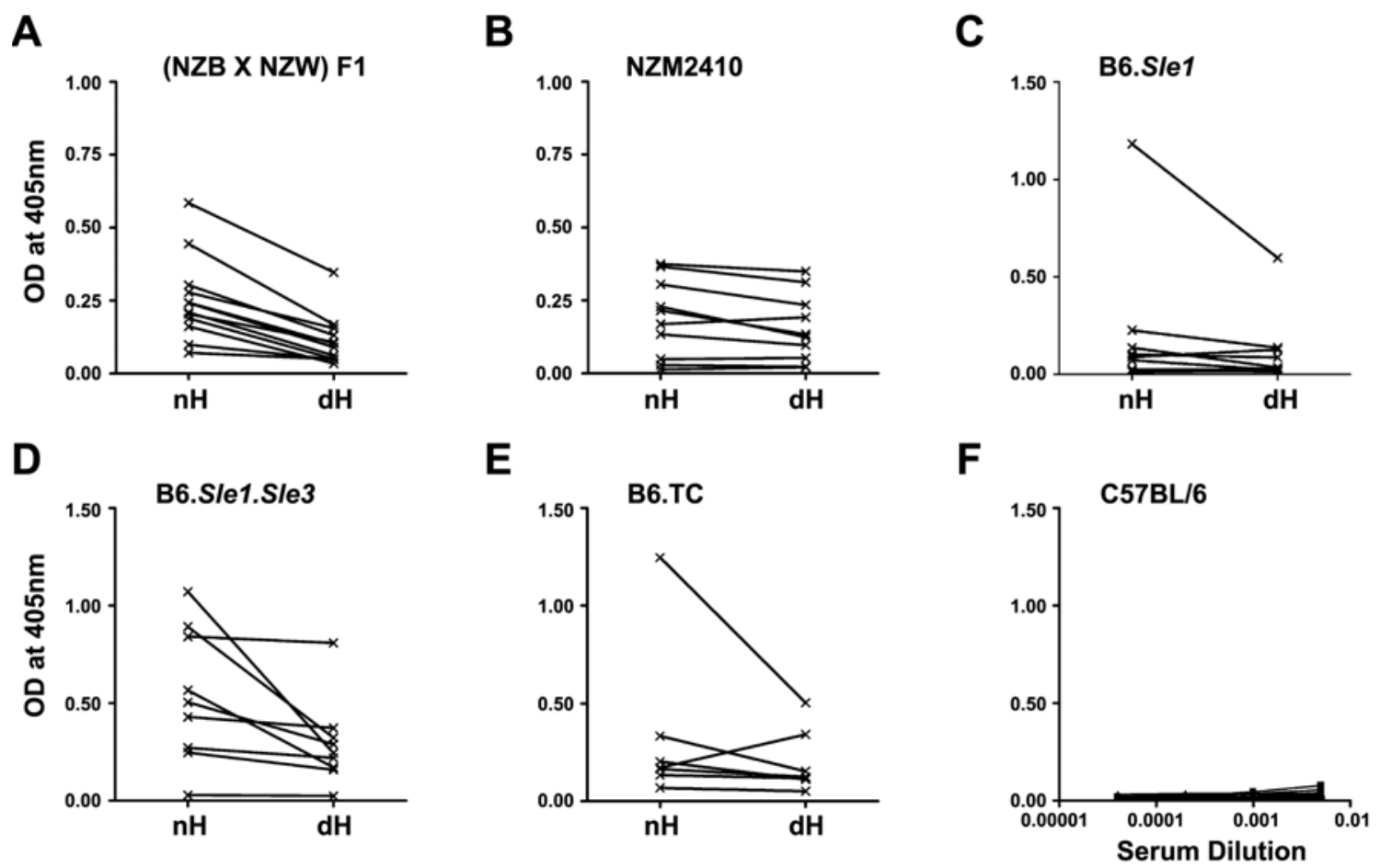

Figure 4-2: Binding of IgG from autoimmune prone and control mice to nondeiminated histones and deiminated histones

Sera from (NZBxNZW)F 1 (A), NZM2410 (B), B6.Sle1 (C), B6.Sle1.Sle3 (D), B6.TC (E) and control $\mathrm{C} 57 \mathrm{BL} / 6$ mice $(\mathrm{F})$ were tested for $\operatorname{IgG}$ binding to non-deiminated histones $(\mathrm{nH})$ and deiminated histones $(\mathrm{dH})$. Complete binding curves were obtained and OD values for a single dilution were plotted in panels $\mathrm{A}$ to $\mathrm{E}$. We plotted data from 1:1000 dilutions in all other than $\mathrm{B}$ where data is from 1:200 dilution. Absorbance values for each serum corresponding to $\mathrm{IgG}$ binding to $\mathrm{dH}$ and $\mathrm{nH}$ are shown and are connected by a line indicating the pairs of data for the binding of each serum to the two antigens. Significance of the readings was tested by paired, two-tailed T-test. Binding to $\mathrm{dH}$ was significantly less than to $\mathrm{nH}$ for $(\mathrm{NZBxNZW}) \mathrm{F}_{1}(\mathrm{p}<0.0001)$, NZM2410 $(\mathrm{p}=0.031)$ and B6.Sle1.Sle3 $(p=0.032)$. The binding of B6.Sle1 $\operatorname{IgG}(p=0.203)$ and B6.TC $(p=0.278)$ tended to be lower to $\mathrm{dH}$. In comparison, control B6 mice showed negligible binding to either form of histones $(\mathrm{F})$. 
Figure 4-3: Western blot of IgG to deiminated histones and non-deiminated histones

Equal amounts of deiminated histones $(\mathrm{dH})$ and non-deiminated histones $(\mathrm{nH})$ were resolved on SDS-PAGE, transferred to nitrocellulose, and stained with Ponceau red (upper left panel). The stained bands migrating with the mobilities of core and linker histones are indicated along the margins. Strips of membrane containing $\mathrm{nH}$ or $\mathrm{dH}$ were probed with (NZBxNZW)F 1 , NZM2410, B6.Sle1, B6.Sle1.Sle3 and B6.TC sera at 1:500 dilution and developed with anti-mouse IgG-HRP. Autoimmune prone mice sera bound $\mathrm{nH}$ in preference to $\mathrm{dH}$. The experiments were performed at least three times with consistent results. One experiment is shown and was used to derive data for Table 4-1. 


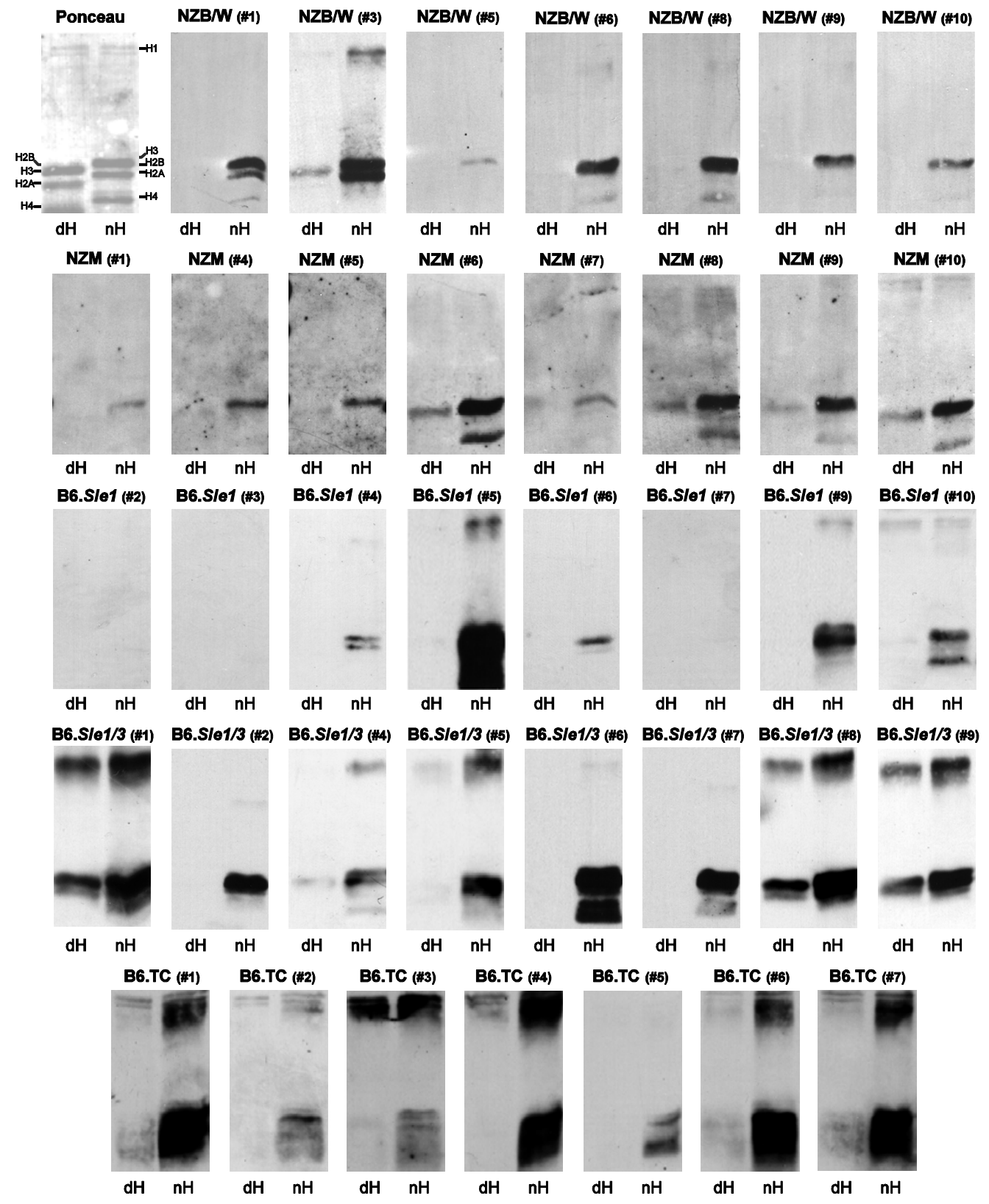


Table 4-1: $\quad$ Summary of Western blot analysis

\begin{tabular}{ccccccc}
\hline $\begin{array}{c}\text { Histone } \\
\text { Subtype }\end{array}$ & $\begin{array}{c}\text { NZWB X } \\
(\mathrm{n}=7)\end{array}$ & $\begin{array}{c}\text { NZM2410 } \\
(\mathrm{n}=8)\end{array}$ & $\begin{array}{c}\text { B6.Sle1 } \\
(\mathrm{n}=8)\end{array}$ & $\begin{array}{c}\text { B6.Sle1.Sle3 } \\
(\mathrm{n}=8)\end{array}$ & $\begin{array}{c}\text { TC } \\
(\mathrm{n}=7)\end{array}$ & $\begin{array}{c}\text { Total } \\
(\mathrm{n}=38)\end{array}$ \\
\hline H3 & $7: 1$ & $8: 4$ & $5: 0$ & $8: 4$ & $7: 0$ & $35: 9$ \\
H2A & $2: 0$ & $1: 0$ & $5: 0$ & $6: 2$ & $7: 0$ & $21: 2$ \\
H4 & $2: 0$ & $4: 0$ & $2: 0$ & $4: 0$ & $6: 0$ & $18: 0$ \\
H1 & $1: 0$ & $1: 1$ & $2: 1$ & $5: 4$ & $6: 5$ & $15: 11$ \\
Total & $12: 1$ & $14: 5$ & $14: 1$ & $23: 10$ & $26: 5$ & $89: 22$ \\
\hline
\end{tabular}

Results are listed as a ratio of IgG binding to non-deiminated histones: deiminated histones. We set $120 \%$ of background intensity as threshold for positive bands. 
plates, as seen by the nearly identical binding of the LG2.2 mAb (data not shown) whose epitope corresponds to first 13 amino acid residues of histone H2B (337).

To examine the preferential binding to non-deiminated histones, we used Western blotting. The stringency of binding was increased by including both SDS $(0.05 \%)$ and NP-40 $(0.5 \%)$ in the binding buffer. Indeed, under these conditions, many of the sera bound exclusively or with preference to non-deiminated histones relative to deiminated histones (Figure 4-3 and Table 4-1). A variety of binding patterns were observed, including exclusive binding to one or two core histones. Binding to histone $\mathrm{H} 3$ was observed most often, whereas binding to $\mathrm{H} 4$ and $\mathrm{H} 2 \mathrm{~A}$ were rare. In addition, binding to a band with the mobility of the linker histone $\mathrm{H} 1$ was observed in several blots. Overall, binding was biased toward non-deiminated histones over deiminated histones (Table 4-1), although some IgG binding to deiminated histones was also observed. To assess the possibility that DNA-anti-DNA complexes present in sera contribute to the observed histone binding, we treated sera with DNase1 prior to incubation with the histone blots. None of the sera tested altered their preferential binding to non-deiminated histones following treatment with DNase1, and the immunoreactivity of DNA-depleted sera was indistinguishable from untreated sera (data not shown). Therefore, anti-DNA antibodies did not measurably contribute to the observed results.

\section{$\left(\right.$ NZBxNZW) $F_{1}$ Splenocytes Respond to Deiminated Histones or Non-deiminated Histones}

To investigate whether splenocytes from autoimmune (NZBxNZW) $\mathrm{F}_{1}$ mice, are capable of responding to deiminated histones or non-deiminated histones, we measured the proliferation of splenocytes from $(\mathrm{NZBxNZW}) \mathrm{F}_{1}$ mice of different ages. At 6 to 10 weeks of age, prior to any measurable anti-DNA reactivity, the splenocytes did not proliferate in response to either form of histone (Figure 4-4A and B). At 20 to 21 weeks of age, anti DNA autoantibodies could be detected in the sera, and splenocytes proliferated in response to non-deiminated histones and deiminated histones (Figure 4-4C and D). Thus, splenocyte responses to histones arose in parallel with humoral

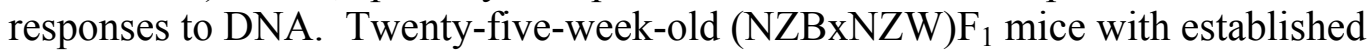
autoimmunity showed splenocyte proliferation in response to deiminated histones and non-deiminated histones (Figure 4-4E and F), suggesting the presence of histonereactive $\mathrm{T}$ cells in the spleens of autoimmune mice. Although some mice showed a tendency to preferentially respond to deiminated histones, others preferred nondeiminated histones. Notably, preference could switch, depending on the length of stimulation (Figure 4-4E and F), suggesting that growth characteristics of some splenocytes may be influenced by deimination. This suggested that $\mathrm{T}$ cell help for $\mathrm{B}$ cells with receptors to non-deiminated histones and deiminated histones should be available following the development of autoimmunity in these mice. Interestingly, splenocytes from BALB/c mice immunized with deiminated histones proliferated vigorously in response to deiminated histones yet failed to respond to non-deiminated histones (Figure 4-5). Yet, B cells from BALB/c mice immunized with either non-deiminated histones or deiminated histones, with or without aTLR7 agonist, showed no response after multiple 

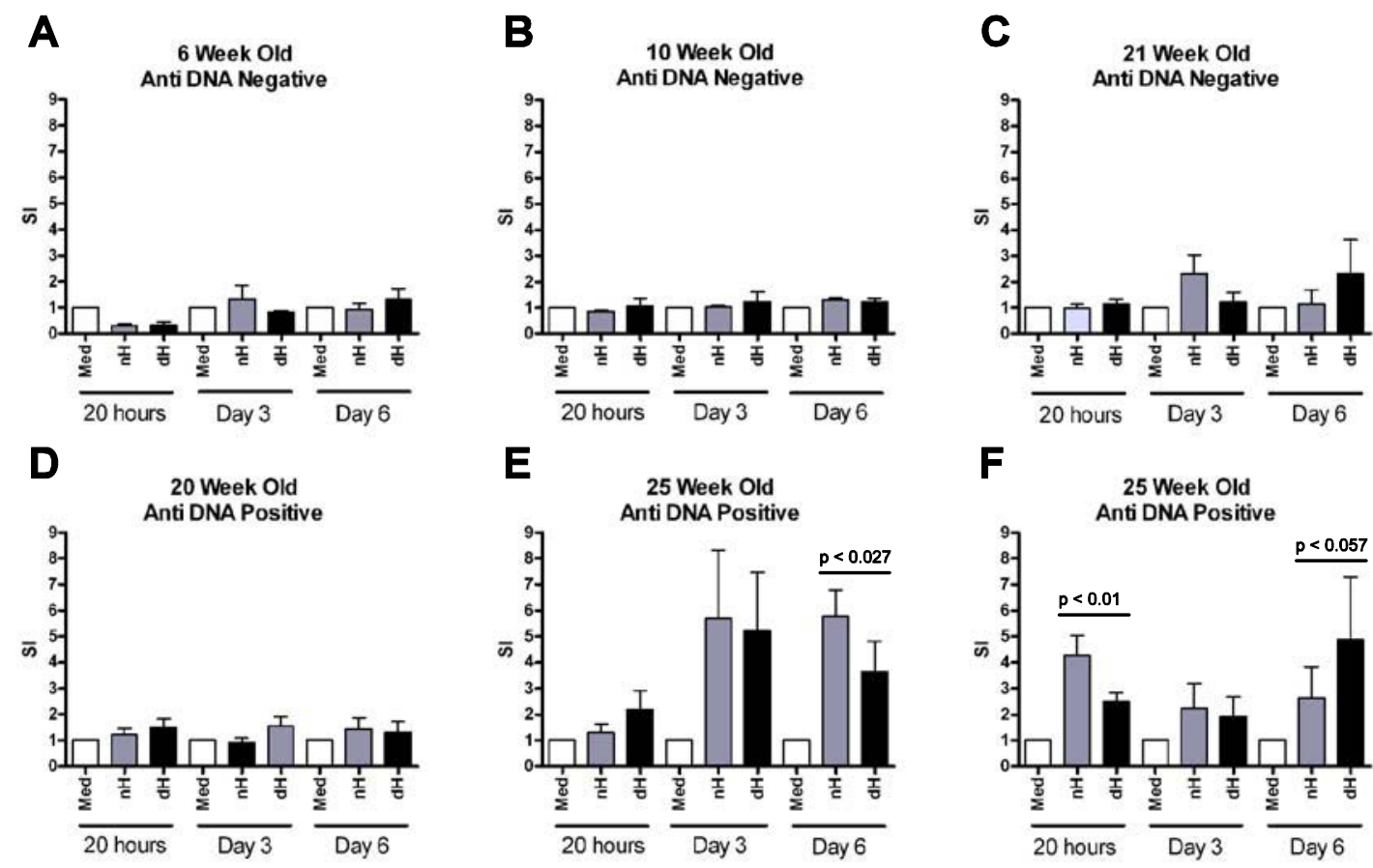

Figure 4-4: $\quad$ Proliferation of splenocytes from $(\mathrm{NZBxNZW}) \mathrm{F}_{1}$ mice

Splenocytes derived from (NZBxNZW)F $\mathrm{F}_{1}$ mice of different ages were tested for dsDNA binding by ELISA (A-F). In parallel, splenocytes were stimulated with deiminated histones $(\mathrm{dH})$ or non-deiminated histones $(\mathrm{nH})$ in vitro, as indicated. Proliferation was assessed following 20 h, 3 days and 6 days in culture, and the response was determined by $\left[{ }^{3} \mathrm{H}\right]$ thymidine incorporation. The results are presented as stimulation indices (SI) that were calculated from mean cpm values of triplicate wells. The significance of differences between samples was determined by unpaired T-test and the $p$ values are indicated. 


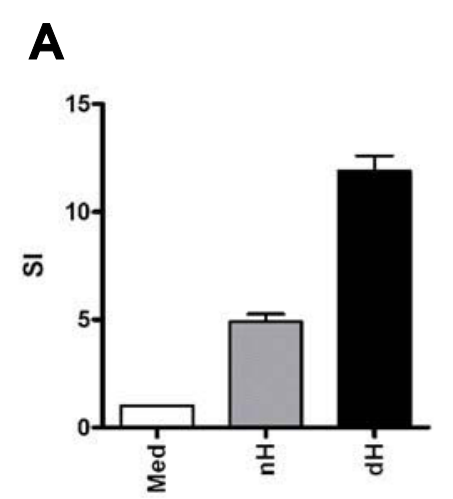

dH Immunized
B

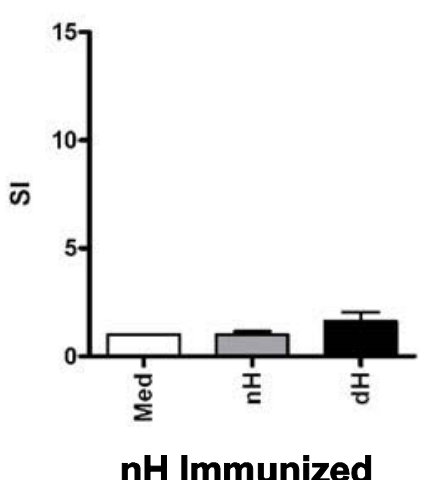

C



Ova Immunized

Figure 4-5: Proliferation of splenocytes from immunized BALB/c mice

Splenocytes from mice immunized with deiminated histones (A) or non-deiminated histones (B) were tested for proliferation in response to deiminated histones $(\mathrm{dH})$ or non-deiminated histones $(\mathrm{nH})$. Two mice were immunized with OVA (C) as control. The results are presented as stimulation indices (SI) that were calculated from mean $\mathrm{cpm}$ values of triplicate wells incubated with respective antigens for $72-96$ hours. A good proliferation response to deiminated histones was seen in splenocytes from mice immunized with deiminated histones, whereas splenocytes from mice immunized with non-deiminated histones showed low to negligible proliferation. 
immunizations (Figure 4-6).

\section{Spleens of Autoimmune Mice Have Increased Levels of Deiminated Histones}

In autoimmunity, the chronic inflammation may be expected to induce PAD4 activation and histone deimination. To test for the presence of deiminated histones in tissues of autoimmune mice, we dissected tissues from B6.TC mice and littermate controls. Lysates of bone marrow, spleen, liver and kidney were probed for deiminated histone $\mathrm{H} 3$ and total $\mathrm{H} 3$. Out of the 7 B6.TC mice tested, 6 showed detectable amounts of deiminated histone $\mathrm{H} 3$ in their bone marrow and spleen (Figure 4-7A and $\mathbf{B}$, see box). The spleens of B6.TC mice had markedly increased levels of deiminated histone $\mathrm{H} 3$ as compared to C57BL/6 controls or control cell lysate (Figure 4-7). The overall amounts of histone $\mathrm{H} 3$ were similar in all samples, as indicated by the equivalent immunoreactivity of an anti- $\mathrm{H} 3$ antibody that does not discriminate deiminated histone $\mathrm{H} 3$ from non-deiminated histone $\mathrm{H} 3$. Therefore, the bone marrow and spleens of autoimmune B6.TC mice contained increased quantities of deiminated histone H3. In addition, the kidneys of one autoimmune mouse exhibited increased levels of deiminated histone H3 (Figure 4-7C, \#12). Unexpectedly, the bone marrow, spleen and kidney of one C57BL/6 mouse showed elevated levels of deiminated histone H3 (Figure 4-7A, B and $\mathbf{C}, \# 2$ ). Yet, this C57BL/6 mouse showed no binding to either non-deiminated histones or deiminated histones on ELISA (data not shown).

\section{Discussion}

In this study, we observed that in autoimmune mice, B cells readily produce $\operatorname{Ig} G$ autoantibodies to non-deiminated histones (Figure 4-2 and 4-3), suggesting the availability of T cell help. However, autoimmune mice exhibited a striking B cell bias for non-deiminated histones over deiminated histones. Of all the mice tested by ELISA, only one B6.TC showed preferential binding to deiminated histones (Figure 4-2E). On Western blot this serum differed from most others in that it showed equal binding to deiminated and non-deiminated $\mathrm{H} 1$ while the binding to core histones was reduced. In 35 of 35 mice that made anti-histone $\mathrm{H} 3 \mathrm{IgG}$, preference was invariably in favor of nondeiminated histone H3 (Figure 4-3 and Table 4-1). In 19 animals, we failed to detect antibodies to deiminated histones by immunoblotting, whereas antibodies to nondeiminated histones were readily observed (Figure 4-3). Strongly biased binding to nondeiminated histones indicates that autoantibody epitopes often (or exclusively) depend on arginines that are substrates for PAD4. This is surprising, as other, non-arginine containing epitopes should also be accessible for antibody binding. These results indicate that, even in overt autoimmunity, IgG binding to deiminated histones is suppressed, and autoantibody binding is focused on arginine-containing epitopes that are absent from deiminated histones. Deimination itself is unlikely to interfere with antibody binding to deiminated histones because autoantibodies to deiminated histones are prevalent in patients with Felty's syndrome (303). One possible explanation for binding to nondeiminated histone epitopes in preference over deiminated histones is that deiminated 

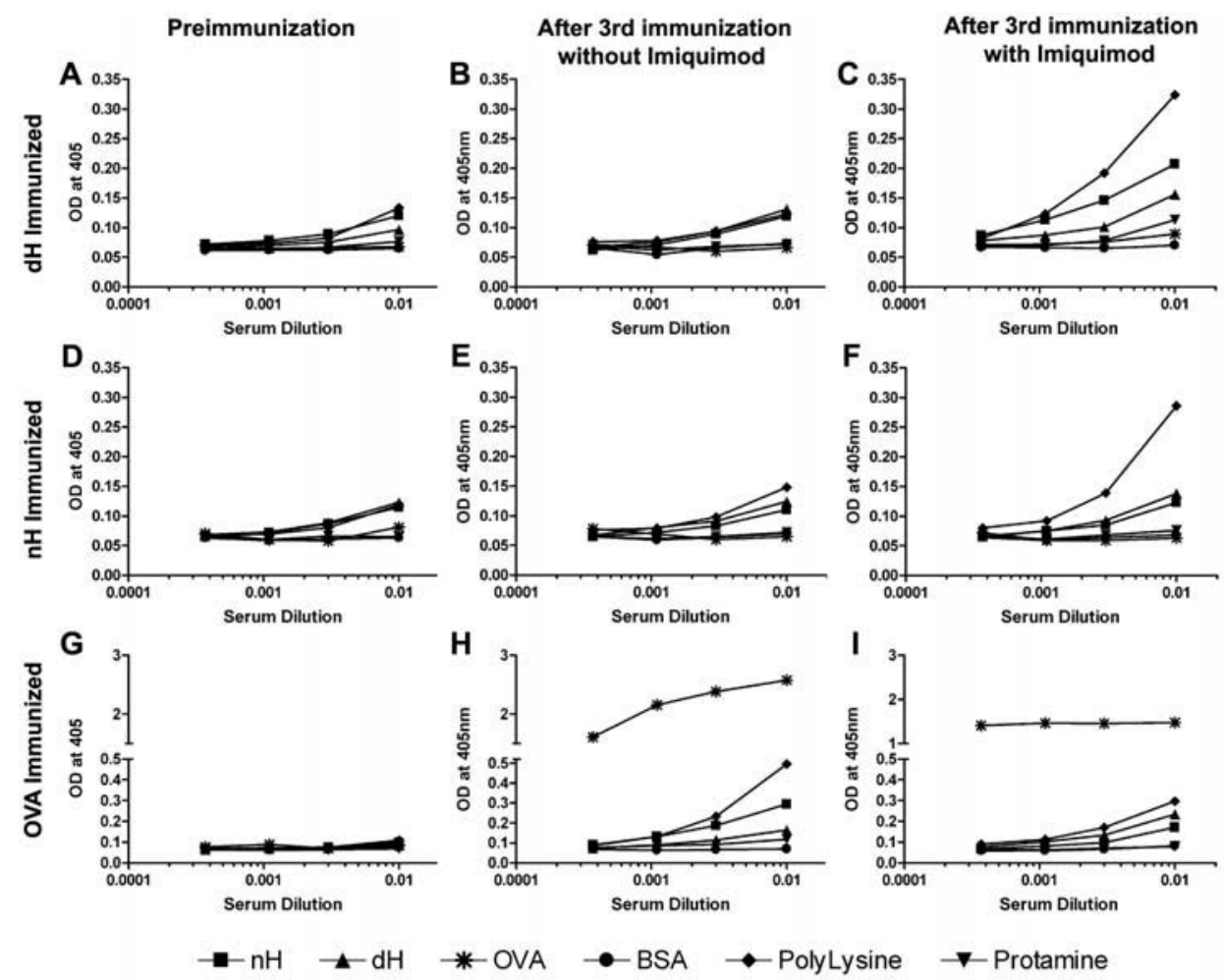

Figure 4-6: B cell response after $3^{\text {rd }}$ immunization using Imiquimod, a TLR-7 agonist as adjuvant

Sera from pre-immunized mice (A, D, G) as well as sera after the third immunization with imiquimod $(\mathrm{C}, \mathrm{F}, \mathrm{I})$ or without $(\mathrm{B}, \mathrm{E}, \mathrm{H})$ were tested against the antigens in the legend. Reactivity to antigens was unchanged before and after immunization in mice immunized with deiminated histones (A and B) and non-deiminated histones (D and E). With imiquimod as adjuvant, the response to deiminated histones or non-deiminated histones was not enhanced, but the binding to poly-L-lysine increased (C and F). The response of ovalbumin (OVA) immunized mice to OVA increased after immunization, as expected (H and I). 


\section{A. Bone Marrow}
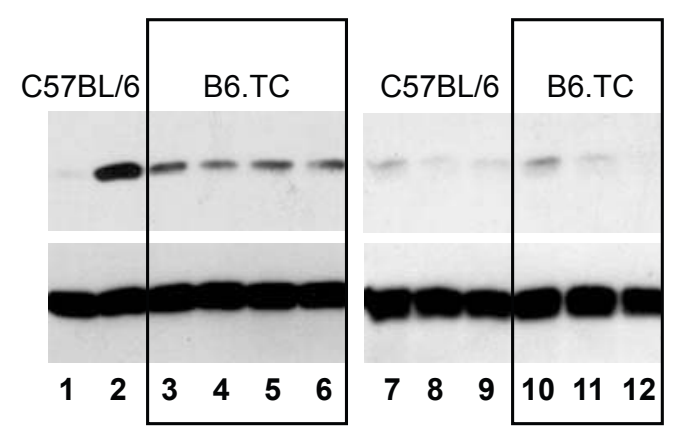

C. Kidney

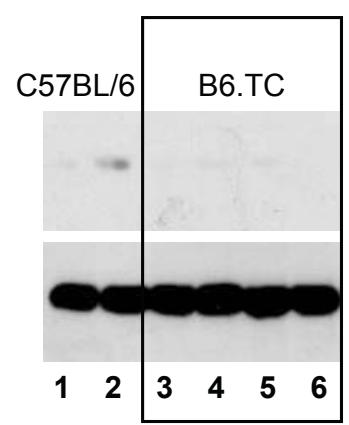

\section{B. Spleen}

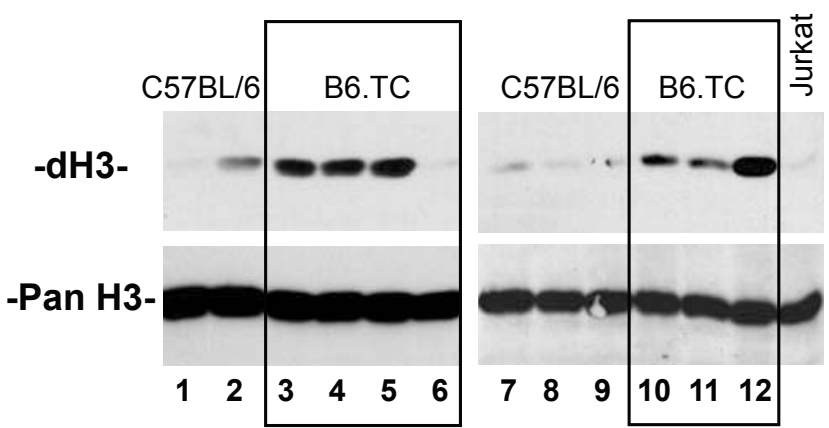

B. Liver

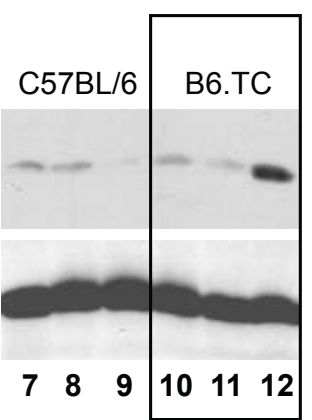

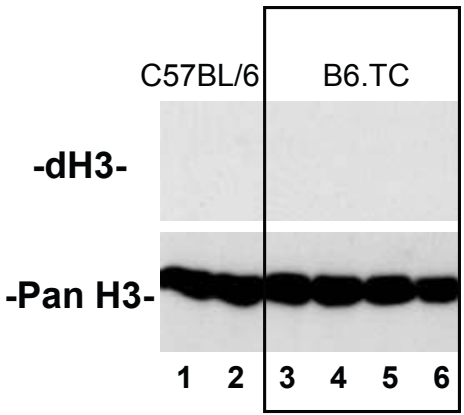

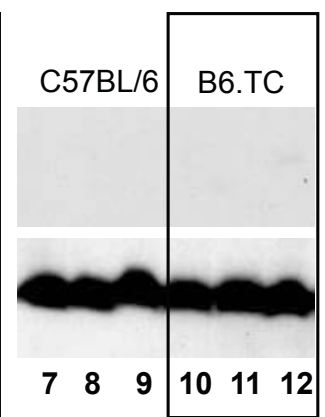

Figure 4-7: Western blot detection of deiminated histones $\mathrm{H3}$ in mouse tissues Equal amounts of freshly prepared lysates from bone marrow (A), spleen (B), kidney (C) and liver (D) of autoimmune B6.TC and control C57BL/6 mice were blotted onto nitrocellulose membranes and probed with anti-deiminated histone $\mathrm{H} 3(\mathrm{dH} 3)$ antibody. A total of 12 mice were analyzed in two separate blots and same tissues are grouped together for presentation. Lanes corresponding to B6.TC tissues are highlighted in box. As control Jurkat lymphoma cells were used (B). The same blots were also re-probed with an antibody that recognizes total histone H3 (Pan H3) to confirm equal loading of lysates. Bone marrow and spleen of 6 out of 7 B6.TC mice showed presence of deiminated histone $\mathrm{H} 3$. 
histones remain effective tolerogens even after development of autoimmunity.The striking bias toward non-deiminated histones binding was particularly evident in animals containing the lupus susceptibility locus Sle3 (Figures 4-2 and 4-3). As established by Mehrad et al. (53), neutrophils from Sle3 congenics respond more vigorously to bacterial infection and release MPO by degranulation. In response to bacteria or other inflammatory stimuli, neutrophils release deiminated chromatin from the cell to the extracellular milieu $(202,203)$. Deimination indeed may be required for chromatin decondensation that accompanies extracellular chromatin release (213). It has been argued that microbes become entangled in this released chromatin and that they are killed by MPO and other bactericidal enzymes that associate with the chromatin (201). Therefore, this chromatin may act as a neutrophil extracellular trap (NET) that serves to contain and destroy microbial pathogens (194). These findings suggest that NETs are important adaptations of innate immunity and that NETosis represents a specific form of programmed cell death that is morphologically, mechanistically and functionally distinct from apoptosis (196). The data of Mehrad et al. (53) suggest that genes in the genetic interval of Sle3 favor neutrophil activation and subsequent NET chromatin release.

To test the hypothesis that deiminated histones act as tolerogens in autoimmune mice, we prepared lysates from tissues of B6.TC and control mice and analyzed them for deiminated histone $\mathrm{H} 3$. Spleens and to a lesser degree the bone marrow from 7-mo-old female B6.TC mice generally exhibited elevated levels of deiminated histone $\mathrm{H}$. The kidney from these mice, as well as tissues from control mice, had reduced or background levels of deiminated histone H3 (Figure 4-6). Interestingly, kidney lysates from one of the autoimmune mice showed elevated levels of deiminated histones, although it is not known whether the increase in deiminated histones was correlated with any aspect of the pathogenic process in this mouse.

Our observations suggest a tolerance mechanism that may operate in the spleens of autoimmune mice. Autoimmune mice may generate a chronic inflammatory milieu in which neutrophils are more likely to become activated. In these mice, neutrophils may induce histone deimination and become sequestered in the spleen. The clearance of activated neutrophils through the splenic reticuloendothelium is consistent with the observed migration of neutrophils to the light zone in the spleen of LPS-treated mice (338). Detection of deiminated histone $\mathrm{H} 3$ in the spleens of autoimmune mice is consistent with the release of NETs (202). Should chromatin containing deiminated histones be externalized in the spleen, serum nucleases such as DNase1 may degrade it into separate nucleosomes. The effect of the solubilized nucleosomes may be to tolerize splenic B cells that express receptors for anti-deiminated histone epitopes. The only antihistone reactive $\mathrm{B}$ cells escaping this tolerogen would be those that bind non-deiminated histones epitopes, as was observed in our study.

Induction of splenic tolerance by soluble antigen is consistent with earlier studies demonstrating the induction of tolerance in germinal center B cells $(339,340)$. Mice harboring anti-hen-egg-lysozyme B cells respond to an injection of soluble antigen by rapid apoptosis of centrocytes (340). The presence of deiminated histones in the spleens of autoimmune mice together with the B cell response targeting non-deiminated histones 
suggests that deiminated histones act as effective tolerogens in the spleens of autoimmune mice. If so, the ability of soluble nucleosomes to establish tolerance may be beneficial in immune responses to microbial infections. Because NET-ted microbes may be engulfed by antigen presenting cells, it may be vital for the organism to respond to bacteria that become entangled in NETs, while repressing a response to NET/chromatin Ags. Therefore, it is meaningful to predict that NETs act as signals to enhance the immune response to infections, and that effective tolerance mechanisms exist to prevent "collateral" responses to NET chromatin. If peripheral tolerance to deiminated histones is preserved in autoimmune mice, our results may suggest ways to re-establish tolerance in overt autoimmunity.

\section{Conclusion}

Autoimmune disorders are associated with ongoing inflammation. Our results show that autoantibodies from lupus prone mice preferentially recognize non-deiminated histones over deiminated histones. Consistent with inflammatory stimuli causing histone deimination in neutrophils, we found high levels of deiminated histone $\mathrm{H} 3$ in bone marrow and spleen of lupus prone mice. We conclude that the biased autoantibody response to non-deiminated histones seen in lupus mice, in spite of the availability of deiminated histones antigen indicates effective tolerance against deiminated histones. 


\section{CHAPTER 5. DISCUSSION}

Despite decades of intense research, we do not know the stimuli that trigger the production of autoantibodies in systemic autoimmune diseases (341). The autoantibodies themselves have been studied and found to be useful as diagnostics for different autoimmune diseases. For example, autoantibodies reacting against nuclear antigens, most notably DNA and histones, are a distinguishing feature of systemic lupus erythematosus (SLE), whereas autoantibodies to a citrullinated cyclic peptide (CCP) are a reliable diagnostic for rheumatoid arthritis (RA). A plausible prediction is that the careful analysis of autoantibodies will lead to a better understanding of their origins.

The structure of autoantibodies argues that they are selected for improved binding to autoantigens. The genetic mechanisms that give rise to autoantibodies argue strongly in favor of this conclusion. Autoantibody sequences indicate that even though diverse $\mathrm{V}$ genes code for anti-DNA and rheumatoid factor (RF) autoantibodies in murine models of SLE and RA, junctional diversity and somatic mutations make essential contributions to binding (342-344). In consequence, only few B cell clones secure conditions for expansion. The expanded autoreactive B cell clones participate in antigen presentation, given that $\mathrm{T}$ cell help promotes antibody $\mathrm{H}$ chain isotype switching and selects for mutations that improve antibody binding to autoantigens. As result, autoantibodies acquire all of the molecular hallmarks of affinity maturation, just as antibodies to foreign antigens do (345). Therefore, the identification of molecular features of autoantigens that drive systemic autoimmune diseases holds the key for understanding the pathways that stimulate the production of autoantibodies.

\section{Post-Translational Modification of Autoantigens}

Autoantibodies frequently target post translational modifications (PTM) in selfantigens $(64,65)$, including PTM that are introduced during apoptotic cell death (346, 347). Thus, PTMs may provide a mechanism for converting innocuous self components into targets of an autoimmune response. PTMs generate "neo- epitopes" that alter antigen processing and presentation (348). One such PTM is the enzymatic conversion of arginines in a polypeptide chain to citrullines. Citrullination, also called deimination, is the work of peptidylarginine deiminases (PADs). PADs are $\mathrm{Ca}++-$ dependent enzymes that are conserved in all vertebrates $(204,205)$. The conserved nature of PADs implies an important physiological role. Indeed, citrullination regulates zygotic development, the myelination of neurons, and properties of the epidermis.

PAD4 is the only PAD that has a nuclear localization signal and deiminates histones H2A, H3, and $\mathrm{H} 4(268,327)$. A link exists between PAD4 haplotypes and risk of RA. Alleles linked to an increased susceptibility to RA encode a more stable PAD4 mRNA than alleles that are neutral for RA (228). The increased mRNA stability may lead to increased expression of PAD4 in individuals bearing the susceptible haplotype. An association between deiminated proteins and autoimmune diseases strongly suggests 
that deimination generates novel epitopes that are able to break tolerance and initiate autoimmune responses.

\section{PAD4 Action in Neutrophils}

PAD4 is most abundantly expressed in granulocytes (349), monocytes (350) and mast cells (310). PAD4 may associate with cytoplasmic granules, but it also enters the nucleus where it may participate in gene regulation by deiminating histones (268). Deimination transforms a positively charged amino acid (arginine) into a neutral amino acid (citrulline), resulting in the net loss of one positive charge per modified residue (206). Therefore, deimination changes not only the primary sequence of a protein but also its net charge. During the reaction, the guanidino group of arginine is attacked by a Cys residue of PAD4, resulting in the formation of a tetrahedral adduct (351). A

nucleophilic attack by a water molecule then cleaves the adduct to form the keto group of citrulline, while releasing ammonia and regenerating the Cys residue of the enzyme (206, 207). The net result is the conversion of arginine to citrulline with a mass shift of $+1 \mathrm{Da}$.

The activation of PAD4 in neutrophils is exquisitely sensitive to inflammatory stimuli, such as f-MLP, LTA or LPS (202). In contrast, the induction of neutrophil apoptosis blocks PAD4 activation (202). Whereas senescent neutrophils that were not needed to fight an infection die by apoptosis $(352,353)$, neutrophils faced with microorganisms in the course of an infection perform an elaborate form of cell suicide. Those neutrophils die by ejecting nuclear chromatin in order to capture, or "trap", microbial pathogens (194). PAD4 activity precedes and facilitates the release of chromatin from neutrophils (203). In fact, PAD4 is essential for neutrophil extracellular trap (NET) release and, in the absence of PAD4, the innate response to bacterial infections is impaired (213). Since the discovery that neutrophils release chromatin in an innate response to infections (194), NET release has been reported in responses to bacterial and fungal infections. NET release is triggered by neutrophil encounter with Staphylococcus aureus (194) or Mycobacterium tuberculosis (354) and in response to exposure to Candida albicans (195) and Aspergillus fumigatus $(355,356)$. NETs function in trapping and damaging microorganisms and this explains how extracellular nucleases produced by bacteria function as virulence factors (309). The release of NETs is linked to various clinical complications of infectious diseases, including bacterial sepsis $(357,358)$ and the increased risk of thrombosis (196). Interestingly, more serious infections and blood clots are known as complications in a number of autoimmune disorders (359). These complications could be linked, in part, to the inopportune release of NETs from autoimmune patient neutrophils.

Clearly, NETs deserve wider experimental scrutiny because, in infections (285), or in response to adjuvants (312), NETs connect citrullinated histones with strong immune stimuli. This feature of NETs could be instrumental in breaking tolerance and inducing an autoimmune response to chromatin antigens. 


\section{Neutrophil Extracellular Traps and Autoimmunity}

That NETs may stimulate autoimmunity follows from discoveries that autoantibodies target various NET components. Autoantibodies from patients with systemic autoimmunity may recognize innate immune defense proteins that associate with NETs. This is the case for the major NET components, including the granule proteases elastase, cathepsin $\mathrm{G}$ and proteinase $3(235,236,238)$, as well as for various bactericidal peptides that are stored in granules and co-purify with NETs, such as neutrophil defensins and the LL37 peptide $(239,240)$. Separate studies indicate an increased abundance of activated neutrophils and increased serum levels of neutrophil granule contents in patients with more advanced disease $(235,287)$. In addition, the severity of lupus may also correlate with the levels of autoantibodies to NET components (243). Thus, lupus autoantibodies target NET-associated proteins in addition to the NETs' structural scaffold, DNA and histones.

Other studies directly point to neutrophil activation as a key factor in the stimulation of autoantibodies. Notably, SLE patients possess neutrophils that are prone to NET release (241). The exacerbated response of neutrophils to lupus autoantibody complexes was discovered in studies that used cells from juvenile lupus patients (241). In turn, NETs may persist for a longer time in autoimmunity, as SLE patients' anti-DNA antibodies protect NETs from degradation by nucleases (243). Together, these findings suggest that, in SLE, the release of NETs may be more easily triggered by stimuli, NETs may induce the production of anti-NET antibodies, and the NET antigens may persist in tissues because they are protected by autoantibodies. Thus, NET antigens and anti-NET autoantibodies may be linked in a pernicious pathogenic cycle.

\section{Autoantibodies to Deiminated Histones}

To initiate our search for autoantibodies to deiminated histones, we collected sera from patients with SLE and RA. In addition, we obtained sera from patients with Felty's syndrome (FS), a disorder that shares features of SLE and RA and is defined by the triad of severe arthritis, splenomegaly and neutropenia. FS arises in 1-3\% of RA patients after an extended period of incompletely mitigated disease $(254,360)$. FS patients experience

severe destructive arthritis and develop extra-articular manifestations including rheumatic nodules and vasculitis (361). Neutropenia is thought to be associated with an intense and ongoing autoimmune reponse to neutrophil surface antigens.

Experiments suggest that neutropenia in FS follows from autoimmune etiology rather than a production defect. In mice infused with the sera or isolated immune complexes of FS patients, neutrophil counts drop precipitously, suggesting autoimmunity as the mechanism (362). Both immune complexes and soluble antibodies from FS patients bind neutrophils and mediate their sequestration in the spleen (363). The spleen of FS patients exhibits an expansion of red pulp, sinus hyperplasia, macrophage infiltration, and prominent germinal centers (364). These hallmarks of increased splenic activity indicate that clearance of activated neutrophils by the spleen may promote FS. 
Interruption of the self-sustaining autoreactivity by removal of the patient's spleen restores neutrophil counts, arguing that the spleen provides a sink for neutrophils in FS (364). Thus, it is reasonable to argue that autoantibodies against neutrophil antigens in FS drive neutropenia by splenic sequestration of neutrophils. To understand FS, it will be important to identify the neutrophil targets of autoantibodies.

There are three observations that suggest histone deimination during neutrophil activation as a possible pathogenic mechanism in FS. First, FS patients score positive for binding to the cyclic citrullinated peptide, a screening test that identifies $77 \%$ of FS patients $(365,366)$. Second, a remarkably high percentage of FS patients have autoantibodies to histones, and reports indicate that over $80 \%$ of FS sera contain antihistone IgG (246). Third, there is sequestration of activated neutrophils in the spleen that may, over time, induce autoantibody production against neutrophil antigens. Because we hypothesized that stimulation of neutrophils in FS would lead to PAD4 activation and histone deimination, and because FS patients make anti-histone antibodies, we decided to test FS patient sera for IgG to deiminated histones.

To test for the presence of antibodies to deiminated histones, we reacted histones from calf thymus with recombinant PAD4 to completion. To compare sera from different autoimmune disorders (FS, RA and SLE), tests were performed at equal IgG concentrations. ELISA for deiminated and non-deiminated histones revealed that majority of FS sera contained IgGs that bound deiminated histones with preference over non-deiminated histones. In contrast, all 37 RA sera, except 2 , showed nearly equal binding to deiminated and non-deiminated histones. Using Western blots, we probed the binding of antibodies to individual histones. Deiminated H3 was the major target autoantigen, although binding to other core histones was also observed. In some cases, IgG preferentially bound non-deiminated H2A, H4 and linker histone H1.

Overall, ELISA and Western blot results were in agreement, although some serum samples showed preferred binding to deiminated histones by only one of the methods. The difference arose because of strong binding of antibodies to both antigens on Western blot was recorded as preferential binding by ELISA. In addition, multiple reactivities could be distinguished by Western blot that could cancel each other by ELISA (e.g. antibodies against deiminated $\mathrm{H} 3$ and non-deiminated $\mathrm{H} 4$ ). In one case, an SLE serum showed remarkable preference for deiminated linker histone $\mathrm{H} 1$ by Western blot, yet ELISA showed no preferential binding because H1 was only a minor component of the calf thymus core histone preparation used in coating the plates. Western blots also detected slight preference for deiminated $\mathrm{H} 3$ in several RA samples. We concluded that this weak binding was due to cross-reactivity between antibodies to CCP and citrulline residues on deiminated $\mathrm{H} 3$ (367).

The difference between FS and RA samples is remarkable and argues that neutropenia in FS may be activation-induced neutropenia. Neutropenia is also a known complication of SLE [85], suggesting why some SLE sera showed preference for deiminated histones. Autoantibodies to deiminated histones, an antigen that is produced during neutrophil activation represents strong evidence that NETs contribute to 
autoimmunity in FS. As neutrophils are involved in responses to infections, our findings suggest a novel path whereby sustained infection may lead to autoimmunity. Neutrophil activation and NET formation are also induced by inflammatory cytokines, suggesting how one might arrive at a positive feedback loop that is the hallmark of autoimmune disorders.

\section{Lupus Mice Do Not Make Antibodies to Deiminated Histones}

As (NZB X NZW)F1 mice make anti-chromatin autoantibodies and have higher levels of Type 1 IFN (368) that predispose for NET formation (241), we tested sera from (NZB X NZW)F1 mice and the recombinant inbred New Zealand Mixed NZM2410 strain for the presence of anti-deiminated histone antibodies. We also tested sera from 3 congenic strains that were derived by backcrossing lupus predisposing genetic intervals of NZM2410 mice on a B6 background (369). Studies have shown that the Sle1 genetic interval promotes production of anti-chromatin antibodies $(49,50)$ while the Sle2 interval is responsible for a defect in B cells and renders them hyperactive (51). The Sle3 genetic interval results in hyperactivation of myeloid cells (53). Neutrophils from B6 mice harboring the Sle3 interval respond vigorously to bacteria by degranulation (53). We intended to establish if these autoimmune mice make autoantibodies to deiminated histones by screening sera from these autoimmune mice for reactivity to deiminated histones. The comparison between different mouse sera also could reveal which genetic element, if any, contributes in autoimmune response against deiminated histones. Both ELISA and Western blot demonstrated that spontaneously arising autoantibodies in the autoimmune mice we tested recognize non-deiminated histones over deiminated histones. The preference for non-deiminated histones became more evident on Western blot where almost all the mouse sera bound histones with preference over non-deiminated histones. In 24 out of 35 sera no binding to deiminated histones could be detected, whereas antibodies to non-deiminated histones were easily observed. This argues that B cell responses in autoimmune mice are preferentially targeted against epitopes that contain arginines. For the majority of these sera, deimination of arginine to citrulline reduced binding to histones.

\section{Splenocytes of Autoimmune Mice Respond to Both Deiminated and Non-deiminated Histones}

Splenocytes from (NZB X NZW)F1 autoimmune mice of an age when autoimmunity manifested as indicated by the emergence of anti-dsDNA antibodies proliferated in response to deiminated and non-deiminated histones. Splenocyte proliferation in individual mice was biased to either non-deiminated or deiminated histones indicating that deimination of arginine to citrulline affects recognition of proteins by splenocytes. In individual mice, the proliferation was stimulated more by non-deiminated histones but in some the response later switched to deiminated histones. This suggests that the growth characteristics of some splenocytes may be influenced by deimination. Possibilities for a biased response to deiminated vs. non-deiminated 
histones may include differences in processing and presentation of the two antigens. Proteolytic processing of proteins is a prerequisite for MHC loading. Though many proteases of the lysosomal compartment have overlapping activity, certain proteases are specific for digesting and presenting specific proteins. For example, tetanus toxin $\mathrm{C}$ fragment (TTCF) is specifically cleaved by asparaginyl endopeptidase (248). Consequently, APCs treated with inhibitors of asparaginyl endopeptidase, and not other protease inhibitors, have reduced presentation of TTCF antigen (248). With regard to deiminated histones, cleavage by neutrophil proteases may shift position relative to nondeiminated histones. Neutrophils express cathepsin H (249), a lysosomal cysteine proteinase involed in antigen presentation (250). Cathepsin $\mathrm{H}$ recognizes arginine as its major cleavage site. Neutrophils also express trypsin, a protease that cleaves proteins at carboxy terminal of arginine. Thus, by deiminating arginine to citrulline, the loss of a cleavage site on histones may result in different peptide fragments. Differences in presentation may influence $\mathrm{T}$ cell central tolerance and $\mathrm{B}$ or $\mathrm{T}$ cell activation in the periphery. As splenocytes from (NZB X NZW) F1 mice proliferated in response to both deiminated and non-deiminated histones, we suggest that MHC presentation of deiminated histones is intact in lupus prone autoimmune mice.

The paucity of antibodies to deiminated histones despite the presence of splenocytes that respond to deiminated histones suggests that B cells are tolerant of deiminated histones even in autoimmune mice. Theoretically, the B cell repertoire is very large. It is estimated that $\mathrm{B}$ cells can make receptors of more than $10^{11}$ specificities (370). Thus it is highly likely that B cells from (NZB X NZW) F1, NZM2410 and congenic autoimmune mice have $B$ cells that can recognize histone epitopes with citrullines. The fact that such B cells either fail to develop or are not activated by deiminated histone antigens, or fail to respond even in an inflammatory environment where autoantibodies to non-deiminated histones are readily produced, argues for B cell tolerance to deiminated histones.

$\mathrm{B}$ cell tolerance to self-antigens is induced either during development or in the periphery (371). During development, B cells that express self-reactive BCRs undergo receptor editing which has an increased likelihood of a change in affinity of binding to self-antigens $(372,373)$. B cells that still express a self-reactive BCR are deleted from the repertoire, although notable exceptions have been observed (374). As result of tolerance, a majority of self-reactive B cells can be prevented from populating the periphery. Some self-reactive B cells that escape to periphery are kept in a state of nonresponsiveness or anergy. Essentially contact with self-antigens in absence of engagement by helper T cell or TLR ligands results in B cell anergy (375).

\section{Presence of Deiminated Histones in Lymphoid Organs of Autoimmune Mice}

In search of putative tolerogens, we probed tissue lysates from primary and secondary lymphoid organs of B6.TC congenic with an antibody to deiminated $\mathrm{H} 3$ and compared the results with control C57BL/6 mice. We found that both bone marrow and spleen of B6.TC mice have higher levels of deiminated $\mathrm{H} 3$ than the controls. This 
suggests that B cell tolerance to deiminated histones may be maintained by presence of deiminated histones both at developmental stages and in the periphery. Since we have used whole tissue lysates for our analysis, we do not know the cellular source of deiminated histones or how they are presented to B cells. Overall, our results indicate that high levels of deiminated histones in autoimmune mice negatively influence B cell responses to deiminated histones. How deiminated histones induce tolerance or what precise mechanisms are involved in tolerance to deiminated histones need further investigation.

\section{$B$ and $T$ Cell Responses in BALB/c Mice Immunized with Deiminated Histones}

Immunization with deiminated fibrinogen in DR4-IE transgenic mice was able to induce arthritis characterized by synovial hyperplasia and ankylosis (376). BALB/c mice immunized with deiminated fibrinogen in presence of Freund's complete adjuvant produced an antibody response to deiminated fibrinogen but not to non-deiminated fibrinogen (377). By immunizing BALB/c mice with deiminated histones in the presence of different adjuvants, we tested whether deimination itself is sufficient in inducing antibody responses against histones. Immunization with both deiminated and nondeiminated histones failed to induce antibodies to histones even after multiple immunizations. That either form of histones failed to induce any B cell response in the presence of adjuvants like LPS or Imiquimod, a TLR7 agonist, suggest active tolerance to histones with or without deimination.

The splenocytes of BALB/c mice immunized with non-deiminated histones when incubated with either form of histones showed poor proliferation response similar to that observed with media alone. The splenocytes from mice immunized with deiminated histones showed increased proliferation in response to both deiminated and nondeiminated histones. Previously Hill et al have found that deiminated peptides are presented better by MHC molecules of DR4-IE transgenic mice (224). Similarly, we found increased proliferation response in presence of deiminated histones suggesting that deimination influences antigen processing and presentation to $T$ cells. The fact that mice immunized with deiminated histones, in spite of having splenocytes that may respond to deiminated histones, still showed no B cell response to histones indicate strong B cell tolerance to histones that is not affected by deimination.

\section{Deiminated Linker Histones in Neutrophils}

Linker histones bind linker DNA at entry and exit points of nucleosomes, thus condensing chromatin into a compact structure (378-380). H1 has multiple isoforms all of which have similar structural features; they are composed of a central globular domain flanked by a long lysine rich C-terminal domain tail and a short N-terminal region (381). PTMs, particularly phosphorylation on the C-terminal tail of $\mathrm{H} 1$ during different stages of cell cycles are important for chromatin remodeling (382). The globular domain of H1 also binds chromatin and is involved in protecting it from nuclease digestion $(383,384)$. 
Specific interactions of this domain with nucleosomes may influence the structural geometry of chromatin as well as gene expression (385-387). The globular domain of H1 contains positively charged residues that interact with nucleosomes through two distinct binding sites. Strong contribution to binding at the first site in histone H1.0 comes from residues: His 25, Arg 47, Lys 69, Lys 73, Arg 74 and Lys 85, whereas for second smaller site contribution to binding was by Arg 42, Arg 94 and Lys 97 (318). In all these positions introduction of a negative charge by replacement of one of these residues with glutamic acid reduced binding, while introduction of residues with a positive charge resulted in near similar or higher binding (318). All somatic subtypes of H1 have arginine residues in or near the DNA binding region of globular domain (297). With conversion of positively charged arginine to neutral citrulline deimination results in a net loss of charge that may affect its interactions with linker DNA. Formation of NETs during neutrophil activation requires rapid changes in chromatin structure. Deimination of histones is considered essential for release of NETs as neutrophils deficient in PAD4 are also deficient in making NETs (203). That the primary function of linker histones is to keep chromatin condensed, that deimination is essential for NET release, and that deimination may alter interaction between $\mathrm{H} 1$ and DNA, prompted us to investigate if linker histones in neutrophils are deiminated.

By extracting total RNA from circulating neutrophils we assessed mRNA expression for different $\mathrm{H} 1$ subtypes on a microarray chip. Among the different subtypes H1.4 was highly expressed in neutrophils and its expression levels were about 4-5 fold more than H1.0 that has the second highest expression levels. The expression levels for H1.1, H1.3 and H1.5 were low and below the housekeeping gene HGPRT used as control whereas H1.2 was expressed at intermediate levels between HGPRT and H1.0. We chemically extracted $\mathrm{H} 1$ from neutrophils treated with ionophore in presence of $\mathrm{Ca}++$ and found deimination of $\mathrm{H} 1$ both by probing it with Anti-Cit (modified) Ab by Western blot and also by Q-TOF Mass Spectrometery followed by MSMS where we identified peptides with characteristic +1 Da mass shift of arginine converting to citrulline. We identified deiminated peptides that are unique to $\mathrm{H} 1.0$ and $\mathrm{H} 1.5$ or shared between $\mathrm{H} 1.2$, $\mathrm{H} 1.3$ and H1.4. We also identified peptides with unique sequences corresponding to $\mathrm{H} 1.0, \mathrm{H} 1.2, \mathrm{H} 1.4$ and H1.5 indicating the presence of these $\mathrm{H} 1$ subtypes in neutrophils. With these results we identified linker histone $\mathrm{H} 1$ as a substrate for deimination. How deimination of $\mathrm{H} 1$ affects its interaction with DNA and what role, if any, deimination of $\mathrm{H} 1$ plays in NET formation will require further studies.

\section{Autoantibodies to Deiminated Linker Histones}

As antibodies to $\mathrm{H} 1$ are present in large number of SLE patients, we tested SLE sera for the presence of anti-deiminated histone $\mathrm{H} 1$ antibodies by using in-vitro deiminated recombinant H1.2. Only 2 of 24 SLE samples showed preferential binding to deiminated histone H1.2 over non-deiminated H1.2 with Western blot. One of the SLE serum sample, SLE6, showed more than 8 times preference for deiminated H1.2 over non-deiminated $\mathrm{H} 1.2$ by Western blot and we further used this serum to characterize autoepitope on H1.2. Two synthetic 16 mer peptides containing citrulline in the position 
R32 and R53 of H1.2 were prepared for competition with deiminated H1.2 to binding to SLE6 autoantibodies in Western blot. The assay showed that the peptide R53Cit competes more effectively for binding to deiminated H1.2 than R32Cit, indicating that deimination of Arg53 occurs within the SLE6 epitope. Arg53 of H1.2 shows sequence homology with Arg42 of H1.0, which is known to be a DNA binding residue. Mutation of Arg42 to glutamic acid on H1.0 which shares sequence homology with Arg 53 of H1.2, decreased DNA binding (318). A similar effect can be deduced for its conversion to citrulline that is also neutral in charge.

We also tested serum samples from 20 patients each of Sjogren's syndrome, Scleroderma, and RA for presence of anti-deiminated $\mathrm{H} 1$ antibodies. We also included samples from FS patients that have earlier showed some preferential binding to deiminated calf thymus $\mathrm{H} 1$ in our survey. The FS sera showed slight preference to deiminated H1.2 over non-deiminated H1.2 (binding ratio of deiminated histones/nondeiminated histones: 1.2-1.3) but this was much less than expected from the Western blot using calf thymus histones. This may be due to the fact that antibodies are reacting more to calf thymus histones recognize a $\mathrm{H} 1$ subtype other than $\mathrm{H} 1.2$ or perhaps different adjoining sequences of calf thymus and human $\mathrm{H} 1.2$ are responsible for difference in binding. While testing other autoimmune disorders for the presence of anti-deiminated histone antibodies we found only 1 out of 20 Sjogren's syndrome patients' samples that showed preferential binding of autoantibodies to deiminated H1.2. Rest of the Sjogren's syndrome samples and also the samples from Scleroderma and RA patients did not discriminate between binding to deiminated and non-deiminated histone H1.2. We conclude from our screening that antibodies to deiminated histone $\mathrm{H} 1$ occur rarely in systemic autoimmune disorders.

\section{Inferences from Human and Mice Antibody Response to Deiminated Histones}

Deimination of histones in neutrophils occurs in response to inflammatory stimuli (202) and consistent with this we found elevated levels of deiminated histones in circulating neutrophils of RA patients as compared to controls. Increased neutrophil activation is also reported in SLE patients where increased levels of Type 1 IFN prime neutrophils to make NETs $(240,241)$. Increased levels of neutrophil NETs in SLE patients are associated with release of immunostimulatory self-peptides and nuclear autoantigens (242). When sera from patients with RA, SLE and FS patients were tested, we found that the majority of FS patients have antibodies to deiminated histones, whereas only a small minority of patients in RA and SLE make anti-deiminated histone antibodies. Both RA and SLE patient sera bound histones on ELISA and Western blot but their binding was not much affected by histone deimination. This implies that deiminated residues do not affect the binding of a majority of RA and SLE anti-histone autoantibodies. The preference for deiminated histones by autoantibodies in FS, a disorder characterized by destruction and sequestration of neutrophils in the spleen (364), suggests that deiminated histones released from neutrophils may be involved in the autoimmune process of FS. Although, the majority of RA sera recognize deiminated antigens, in our assay only a few sera showed higher binding to deiminated histones. The 
facts that FS occurs in patients who have long standing RA, that anti-deiminated histone antibodies are found in majority of FS rather than RA patients and that antibodies to other deiminated proteins arise early in RA patients, suggest that while deimination enhances immune reactivity to many proteins, a response to deiminated histones is not easily induced.

Our results from the study of various lupus prone and control mice support the idea of strong tolerance to deiminated histones. On screening sera from lupus prone mice that readily make autoantibodies against chromatin including histones, we found that autoantibodies in these mice have much higher preference to non-deiminated histones than deiminated histones. We found increased levels of deiminated histones in bone marrow and spleen of autoimmune B6.TC mice which come presumably from neutrophils activated with higher Type 1 IFN levels $(241,368)$ together with ongoing autoimmune injury seen in these mice. Although the exact mechanisms of tolerance to deiminated histones are unknown, the fact that we found antibodies to deiminated histones only in FS, a severe variant of arthritis occurring in patients with long standing RA, suggests that unusual circumstances are needed to elicit such autoantibodies. The inability of BALB/c mice to make antibodies to deiminated histones when immunized with excess of deiminated histones suggests that this is indeed the case. There can be serious consequences for health in case tolerance of deiminated histones is compromised, a component of NETs released during innate neutrophil response to infections $(194,285)$. This is evident in case of FS where we found antibodies to deiminated histones and which differs from RA in having severe arthritis, extra-articular manifestations and a high susceptibility to infections due to lower neutrophil counts.

\section{Model of Autoimmune Pathogenesis in Felty's Syndrome}

We propose the following model of FS pathogenesis based on the biological properties of neutrophils, our data on the high incidence of autoantibodies to deiminated histones, and the disease progression in FS (Figure 5-1). The diagram connects the clinical observations that characterize FS, for example the pre-existing arthritis, neutropenia, splenomegaly and an increased incidence of infections, with the neutrophil response to infections and inflammation. In these circumstances, neutrophils induce histone deimination and expel chromatin from the cell. NETs containing deiminated histones, in complex with bacterial adjuvants, are the most likely antigenic trigger to account for the production of autoantibodies to the modified histones. These autoantibodies, similar to other ANCA, may further stimulate neutrophils, thus completing a cycle that is self-sustaining and drives depletion of mature neutrophils. Clearly, our model is simplified and incomplete, as our data from other autoimmune disorders imply. Even though SLE patients may exhibit increased neutrophil activation and neutropenia, we failed to detect a high incidence of antibodies to deiminated histones. Thus, one might suspect that tolerance mechanisms prevent the development of autoantibodies to deiminated histones and only sustained immune stimulation breaks this tolerance. What factors break tolerance to deiminated histones in FS remains to be determined. Nevertheless, the increased severity of FS as compared to RA, with its 


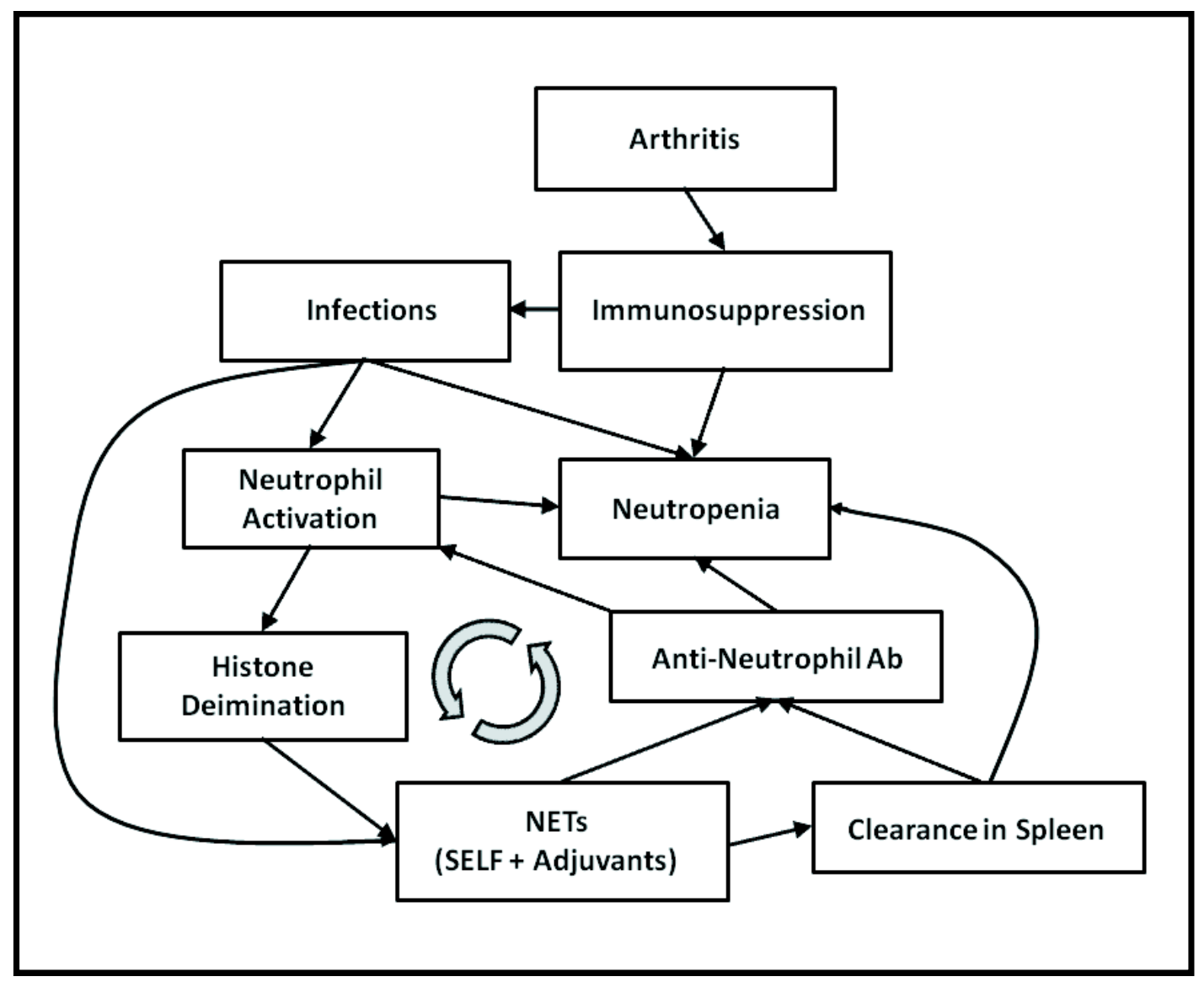

Figure 5-1: A model of autoimmune pathogenesis in Felty's syndrome Diagram shows proposed relationships between clinical manifestations and disease mechanisms. For details, see text. 
rapidly progressing arthritis, extra-articular manifestations and life threatening neutropenia, clearly indicate the need to identify the pathogenic mechanisms of FS.

\section{Future Directions}

An increased expression of PAD4 mRNA is observed in RA patients and they have increased titers of anti-CCP antibodies. Our results that antibodies to deiminated histones are predominantly present in FS patients, a severe variant of RA with splenomegaly and neutropenia; and only infrequently in RA and SLE patients highlight how autoimmunity against deiminated histones contributes to progression of RA to a more severe and crippling form. Anti-deiminated histone antibodies are the first in the group of known ACPAs where the process of deiminated antigen release can be assumed to be activated neutrophils (202). Further research needs to be done to better understand the relationship between neutrophils, PAD4 and autoimmunity. A detailed study is needed to understand how and when these antibodies arise; and how they contribute to disease manifestations. Many studies have found ACPA to be associated with increased severe arthritis in RA. As FS mostly occurs in patients with long standing RA (254, 360), further studies aiming to correlate the emergence of anti-deiminated histones antibodies with onset, duration and progression of FS in RA patients will help to understand autoimmune responses against this deiminated antigen.

Certain MHC haplotypes such as HLA-DRB1*04 associate with ACPA positive RA (388) and studies have shown that deiminated pepties preferentially bind such MHC molecules (224). Research into FS MHC haplotypes associated with anti-deiminated histone antibody response will provide further insights into the development of autoimmunity to deiminated histones. If an association exists between the development of anti-deiminated histone antibodies and particular MHC haplotypes, then it might be a good screening test for identifying RA patients at risk for developing FS. A standardized anti-deiminated histone IgG ELISA may be developed to monitor such patients and RA patients making anti-deiminated histone antibodies might be ideal candidates to for therapeutic intervention with PAD4 inhibitors.

Clinically related to FS is LGL leukemia, in which patients suffer from neutropenia and arthritis similar to, seen in FS $(389,390)$. Such similarities have led many to believe that FS and LGL belong to the same spectrum of diseases and share common pathological mechanism $(255,390,391)$. Because we found that antideiminated histone antibodies are predominantly present in FS patients, we propose that screening LGL patients for these antibodies will be a good way to test whether etiology of LGL is as similar to FS as its clinical features. A key difference between LGL and FS is in the mechanism of neutropenia. In LGL neutropenia is supposed to occur due to production defect as bone marrow become infiltrated by leukemic lymphocytes (392, 393 ) that outcompete myeloid cell precursors or induce apoptosis during development (394). On the contrary neutropenia in FS is due to sequestration of mature activated neutrophils in the spleen $(363,364,395)$. As deimination of histones in neutrophils is seen on activation, we hypothesize that anti-deiminated histone antibodies will be present 
in FS but not in LGL patients. Thus, simple ELISA and Western blot for the presence of these antibodies in LGL patient sera will provide useful information to discriminate between these two disorders.

Another important area of potential research is to investigate and understand the role deimination of histone plays in neutrophil biology. So far the only role suggested for histone deimination is in facilitating neutrophil NETs (203). Yet the mechanism how deimination plays a role in unraveling chromatin is not understood well. With conversion of positively charged arginine to neutral citrulline there is a possibility of decreased interaction between DNA and histones. Careful characterization of interactions between DNA and histones is needed to understand whether this mechanism is involved in the release of NETs. We have also identified that linker histones, responsible to maintain compact chromatin structures in cells, are substrate of PAD4 induced deimination in neutrophils. We identified deimination at Arg 53, a residue reported to interact with DNA. Future studies on the effect of deimination on interactions linker histones and linker DNA will be important in elucidating the mechanism of NET formation.

A related role of deimination in loosening chromatin might be seen while transmigration of neutrophils to the site of inflammation. Neutrophil influx into inflamed sites is preceded by rolling, adhesion to vessel endothelium and then diapedesis through the cell junctions (396). The ability of neutrophils to squeeze themselves through tight junctions of endothelium requires highly plastic nuclei. One possible way to achieve this is by deiminating histones so as to loosen chromatin. The fact that arthritic joints of RA patients are infiltrated with neutrophils (397) and that neutrophils of RA patients show high expression of PAD4 mRNA (228) support this thought. It is possible that increased PAD4 activity allows for increased neutrophil infiltration in joints of RA susceptible individuals and cause increased inflammation in response to even minor trauma. Whether this is true could be ascertained by in vitro transmigration experiments with human donor neutrophil in presence of PAD4 inhibitors or by comparing neutrophil influx after inducing sterile peritonitis in PAD4 deficient and control mice. Presently, neutrophils are known to deiminate histone to make NETs in response to inflammatory stimuli (202). If deimination indeed helps in transmigration of neutrophils then an important question will be to identify a stimulus that induces just sufficient deimination of histones in neutrophils that facilitates transmigration and yet allows normal neutrophil function rather than NET formation. Other interesting questions like what role do deiminated histones play in NETs needs to be investigated in future. Recently, NETs and excess of histones have been shown to promote thrombosis (307). What role deimination plays in increasing thrombosis has not been investigated. As deiminated histones are released during neutrophil activation, it is important to investigate how they interact with other self-proteins.

Numerous reports of autoimmune responses against deiminated non-nuclear proteins have strengthened the notion that deimination, a PTM that accompanies inflammation or differentiation, might lead to autoimmunity by generating 'neoepitopes". In our attempt to understand the contribution of deimination in promoting autoimmunity we found that autoimmune responses against deiminated histones occur 
rarely. Screening of sera from FS SLE, RA, SS, Scl and ANCA vasculides patients revealed that only FS patients have anti-deiminated histone autoantibodies. Other studies have pointed to increased levels PAD4 enzyme and deiminated proteins in RA. Also, SLE patients have preponderance of anti-histone autoantibodies and lupus pathogenesis promotes neutrophil activation and NET formation. Still, our results show that the sera from SLE, RA and many other autoimmune patients do not prefer deiminated histones. Consistent with human studies, sera of autoimmune lupus prone mice preferred nondeiminated histones. While deimination promotes antigen recognition for many nonnuclear proteins, the rarity of autoantibodies against deiminated histones suggests that development of autoimmune responses against important nuclear antigens is protected.

As FS is considered to be a severe variant of RA, the work in this dissertation opens up several interesting questions relevant to understanding of autoimmune process in FS and RA. Studies to identify RA patients at risk of developing FS will help in understanding autoimmune responses against deiminated histones. Our results show robust tolerance to deiminated histones in RA and SLE patients as well as lupus prone mice. Future studies might identify mechanisms of tolerance to deiminated histones which can be applied to modulate autoimmune response against other autoantigens. Our current study also provides opportunities to further investigate neutrophil function of transmigration and NETosis, so that these important mechanisms involved in innate defense can be understood better. Detailed understanding of neutrophil function in health and disease is required to devise better strategies for fighting infections while preventing potential adverse consequences of uncontrolled neutrophils activation such as thrombosis, sepsis and autoimmunity. 


\section{LIST OF REFERENCES}

1. Ehrlich, P., and J. Morgenroth. 1901. Ueber Hämolysine. Berl Klin Wochenschr 38:251-257.

2. Donath, J., and K. Landsteiner. 1904. Ueber paroxysmale Hämaglobinurie. Munch Med Wochenschr 51:1590-1593.

3. Witebsky, E., and N. R. Rose. 1956. Studies on organ specificity. IV. Production of rabbit thyroid antibodies in the rabbit. J Immunol 76:408-416.

4. Rose, N. R., and E. Witebsky. 1956. Studies on organ specificity. V. Changes in the thyroid glands of rabbits following active immunization with rabbit thyroid extracts. J Immunol 76:417-427.

5. Campbell, P. N., D. Doniach, R. V. Hudson, and I. M. Roitt. 1956. Autoantibodies in Hashimoto's disease (lymphadenoid goitre). Lancet 271:820-821.

6. Burnett, F. M., and F. Fenner. 1949. Monograph of the Walter and Eliza Hall Institute of Research in Pathology and Medicine. In The Production of Antibodies 2nd Ed. Melbourne,Macmillan.

7. Wardemann, H., S. Yurasov, A. Schaefer, J. W. Young, E. Meffre, and M. C. Nussenzweig. 2003. Predominant autoantibody production by early human B cell precursors. Science 301:1374-1377.

8. Yurasov, S., H. Wardemann, J. Hammersen, M. Tsuiji, E. Meffre, V. Pascual, et al. 2005. Defective B cell tolerance checkpoints in systemic lupus erythematosus. J Exp Med 201:703-711.

9. Gauld, S. B., R. J. Benschop, K. T. Merrell, and J. C. Cambier. 2005. Maintenance of B cell anergy requires constant antigen receptor occupancy and signaling. Nat Immunol 6:1160-1167.

10. Healy, J. I., R. E. Dolmetsch, L. A. Timmerman, J. G. Cyster, M. L. Thomas, G. R. Crabtree, et al. 1997. Different nuclear signals are activated by the B cell receptor during positive versus negative signaling. Immunity 6:419-428.

11. Lesley, R., Y. Xu, S. L. Kalled, D. M. Hess, S. R. Schwab, H. B. Shu, et al. 2004. Reduced competitiveness of autoantigen-engaged $\mathrm{B}$ cells due to increased dependence on BAFF. Immunity 20:441-453.

12. Yarkoni, Y., A. Getahun, and J. C. Cambier. 2010. Molecular underpinning of B-cell anergy. Immunol Rev 237:249-263.

13. Mueller, D. L. 2010. Mechanisms maintaining peripheral tolerance. Nat Immunol 11:21-27.

14. Goodnow, C. C., J. Sprent, B. Fazekas de St Groth, and C. G. Vinuesa. 2005. Cellular and genetic mechanisms of self tolerance and autoimmunity. Nature 435:590-597.

15. Rose, N. R., and I. R. Mackay. 2006. The Autoimmune Disease 4th Ed. Elsevier Science, San Diego, CA.

16. Jacobson, D. L., S. J. Gange, N. R. Rose, and N. M. Graham. 1997. Epidemiology and estimated population burden of selected autoimmune diseases in the United States. Clin Immunol Immunopathol 84:223-243.

17. Eaton, W. W., N. R. Rose, A. Kalaydjian, M. G. Pedersen, and P. B. Mortensen. 2007. Epidemiology of autoimmune diseases in Denmark. J Autoimmun 29:1-9. 
18. Chan, V. W., F. Meng, P. Soriano, A. L. DeFranco, and C. A. Lowell. 1997.

Characterization of the B lymphocyte populations in Lyn-deficient mice and the role of Lyn in signal initiation and down-regulation. Immunity 7:69-81.

19. Inobe, M., and R. H. Schwartz. 2004. CTLA-4 engagement acts as a brake on CD4+ T cell proliferation and cytokine production but is not required for tuning $\mathrm{T}$ cell reactivity in adaptive tolerance. J Immunol 173:7239-7248.

20. Ueda, H., J. M. Howson, L. Esposito, J. Heward, H. Snook, G. Chamberlain, et al. 2003. Association of the T-cell regulatory gene CTLA4 with susceptibility to autoimmune disease. Nature 423:506-511.

21. Dausset, J. 1981. The major histocompatibility complex in man. Science 213:1469-1474.

22. Vandiedonck, C., and J. C. Knight. 2009. The human Major Histocompatibility Complex as a paradigm in genomics research. Brief Funct Genomic Proteomic 8:379-394.

23. Truog, P., U. Steiger, G. Loewi, and K. Neuhaus. 1975. [HL-A B27 associated rheumatic disease]. Schweiz Med Wochenschr 105:1733-1735.

24. Luthra, H. S., R. H. Ferguson, and D. L. Conn. 1976. Coexistence of ankylosing spondylitis and rheumatoid arthritis. Arthritis Rheum 19:111-114.

25. Khan, M. A. 1995. HLA-B27 and its subtypes in world populations. Curr Opin Rheumatol 7:263-269.

26. Brown, M. A., L. G. Kennedy, A. J. MacGregor, C. Darke, E. Duncan, J. L. Shatford, et al. 1997. Susceptibility to ankylosing spondylitis in twins: the role of genes, HLA, and the environment. Arthritis Rheum 40:1823-1828.

27. Mathieu, A., F. Paladini, A. Vacca, A. Cauli, M. T. Fiorillo, and R. Sorrentino. 2009. The interplay between the geographic distribution of HLA-B27 alleles and their role in infectious and autoimmune diseases: a unifying hypothesis. Autoimmun Rev 8:420-425.

28. Graham, R. R., W. Ortmann, P. Rodine, K. Espe, C. Langefeld, E. Lange, et al. 2007. Specific combinations of HLA-DR2 and DR3 class II haplotypes contribute graded risk for disease susceptibility and autoantibodies in human SLE. Eur J Hum Genet 15:823-830.

29. Fernando, M. M., C. R. Stevens, P. C. Sabeti, E. C. Walsh, A. J. McWhinnie, A. Shah, et al. 2007. Identification of two independent risk factors for lupus within the MHC in United Kingdom families. PLoS Genet 3:e192.

30. Walport, M. J. 2002. Complement and systemic lupus erythematosus. Arthritis Res 4 Suppl 3:S279-293.

31. Holoshitz, J. 2010. The rheumatoid arthritis HLA-DRB1 shared epitope. Curr Opin Rheumatol 22:293-298.

32. Concannon, P., H. A. Erlich, C. Julier, G. Morahan, J. Nerup, F. Pociot, et al. 2005. Type 1 diabetes: evidence for susceptibility loci from four genome-wide linkage scans in 1,435 multiplex families. Diabetes 54:2995-3001.

33. Rich, S. S. 1990. Mapping genes in diabetes. Genetic epidemiological perspective. Diabetes 39:1315-1319.

34. Remmers, E. F., R. M. Plenge, A. T. Lee, R. R. Graham, G. Hom, T. W. Behrens, et al. 2007. STAT4 and the risk of rheumatoid arthritis and systemic lupus erythematosus. N Engl J Med 357:977-986. 
35. Hugot, J. P., M. Chamaillard, H. Zouali, S. Lesage, J. P. Cezard, J. Belaiche, et al. 2001. Association of NOD2 leucine-rich repeat variants with susceptibility to Crohn's disease. Nature 411:599-603.

36. Ogura, Y., D. K. Bonen, N. Inohara, D. L. Nicolae, F. F. Chen, R. Ramos, et al. 2001. A frameshift mutation in NOD2 associated with susceptibility to Crohn's disease. Nature 411:603-606.

37. Begovich, A. B., V. E. Carlton, L. A. Honigberg, S. J. Schrodi, A. P. Chokkalingam, H. C. Alexander, et al. 2004. A missense single-nucleotide polymorphism in a gene encoding a protein tyrosine phosphatase (PTPN22) is associated with rheumatoid arthritis. Am J Hum Genet 75:330-337.

38. Kozyrev, S. V., A. K. Abelson, J. Wojcik, A. Zaghlool, M. V. Linga Reddy, E. Sanchez, et al. 2008. Functional variants in the B-cell gene BANK1 are associated with systemic lupus erythematosus. Nat Genet 40:211-216.

39. Guo, L., H. Deshmukh, R. Lu, G. S. Vidal, J. A. Kelly, K. M. Kaufman, et al. 2009. Replication of the BANK1 genetic association with systemic lupus erythematosus in a European-derived population. Genes Immun 10:531-538.

40. Hom, G., R. R. Graham, B. Modrek, K. E. Taylor, W. Ortmann, S. Garnier, et al. 2008. Association of systemic lupus erythematosus with C8orf13-BLK and ITGAM-ITGAX. N Engl J Med 358:900-909.

41. Barreto, M., E. Santos, R. Ferreira, C. Fesel, M. F. Fontes, C. Pereira, et al. 2004. Evidence for CTLA4 as a susceptibility gene for systemic lupus erythematosus. Eur J Hum Genet 12:620-626.

42. Plenge, R. M., L. Padyukov, E. F. Remmers, S. Purcell, A. T. Lee, E. W. Karlson, et al. 2005. Replication of putative candidate-gene associations with rheumatoid arthritis in $>4,000$ samples from North America and Sweden: association of susceptibility with PTPN22, CTLA4, and PADI4. Am J Hum Genet 77:10441060.

43. Raychaudhuri, S., E. F. Remmers, A. T. Lee, R. Hackett, C. Guiducci, N. P. Burtt, et al. 2008. Common variants at CD40 and other loci confer risk of rheumatoid arthritis. Nat Genet 40:1216-1223.

44. Kariuki, S. N., and T. B. Niewold. 2010. Genetic regulation of serum cytokines in systemic lupus erythematosus. Transl Res 155:109-117.

45. Graham, R. R., S. V. Kozyrev, E. C. Baechler, M. V. Reddy, R. M. Plenge, J. W. Bauer, et al. 2006. A common haplotype of interferon regulatory factor 5 (IRF5) regulates splicing and expression and is associated with increased risk of systemic lupus erythematosus. Nat Genet 38:550-555.

46. Kunz, M., and S. M. Ibrahim. 2009. Cytokines and cytokine profiles in human autoimmune diseases and animal models of autoimmunity. Mediators Inflamm 2009:979258.

47. Rieux-Laucat, F., F. Le Deist, C. Hivroz, I. A. Roberts, K. M. Debatin, A. Fischer, et al. 1995. Mutations in Fas associated with human lymphoproliferative syndrome and autoimmunity. Science 268:1347-1349.

48. Bjorses, P., J. Aaltonen, N. Horelli-Kuitunen, M. L. Yaspo, and L. Peltonen. 1998. Gene defect behind APECED: a new clue to autoimmunity. Hum Mol Genet 7:1547-1553. 
49. Morel, L., C. Mohan, Y. Yu, B. P. Croker, N. Tian, A. Deng, et al. 1997. Functional dissection of systemic lupus erythematosus using congenic mouse strains. J Immunol 158:6019-6028.

50. Sobel, E. S., C. Mohan, L. Morel, J. Schiffenbauer, and E. K. Wakeland. 1999. Genetic dissection of SLE pathogenesis: adoptive transfer of Sle1 mediates the loss of tolerance by bone marrow-derived B cells. J Immunol 162:2415-2421.

51. Mohan, C., L. Morel, P. Yang, and E. K. Wakeland. 1997. Genetic dissection of systemic lupus erythematosus pathogenesis: Sle 2 on murine chromosome 4 leads to B cell hyperactivity. J Immunol 159:454-465.

52. Zhu, J., X. Liu, C. Xie, M. Yan, Y. Yu, E. S. Sobel, et al. 2005. T cell hyperactivity in lupus as a consequence of hyperstimulatory antigen-presenting cells. J Clin Invest 115:1869-1878.

53. Mehrad, B., S. J. Park, G. Akangire, T. J. Standiford, T. Wu, J. Zhu, et al. 2006. The lupus-susceptibility locus, Sle3, mediates enhanced resistance to bacterial infections. J Immunol 176:3233-3239.

54. Vang, T., A. V. Miletic, Y. Arimura, L. Tautz, R. C. Rickert, and T. Mustelin. 2008. Protein tyrosine phosphatases in autoimmunity. Annu Rev Immunol 26:2955.

55. Korman, B. D., D. L. Kastner, P. K. Gregersen, and E. F. Remmers. 2008. STAT4: genetics, mechanisms, and implications for autoimmunity. Curr Allergy Asthma Rep 8:398-403.

56. Ercolini, A. M., and S. D. Miller. 2009. The role of infections in autoimmune disease. Clin Exp Immunol 155:1-15.

57. Mertens, N. M., J. E. Galvin, E. E. Adderson, and M. W. Cunningham. 2000. Molecular analysis of cross-reactive anti-myosin/anti-streptococcal mouse monoclonal antibodies. Mol Immunol 37:901-913.

58. Cunningham, M. W., J. M. McCormack, P. G. Fenderson, M. K. Ho, E. H. Beachey, and J. B. Dale. 1989. Human and murine antibodies cross-reactive with streptococcal $M$ protein and myosin recognize the sequence GLN-LYS-SERLYS-GLN in M protein. J Immunol 143:2677-2683.

59. Hadden, R. D., H. Karch, H. P. Hartung, J. Zielasek, B. Weissbrich, J. Schubert, et al. 2001. Preceding infections, immune factors, and outcome in Guillain-Barre syndrome. Neurology 56:758-765.

60. McClain, M. T., L. D. Heinlen, G. J. Dennis, J. Roebuck, J. B. Harley, and J. A. James. 2005. Early events in lupus humoral autoimmunity suggest initiation through molecular mimicry. Nat Med 11:85-89.

61. Toussirot, E., and J. Roudier. 2008. Epstein-Barr virus in autoimmune diseases. Best Pract Res Clin Rheumatol 22:883-896.

62. Guilherme, L., J. Kalil, and M. Cunningham. 2006. Molecular mimicry in the autoimmune pathogenesis of rheumatic heart disease. Autoimmunity 39:31-39.

63. Getts, M. T., and S. D. Miller. 2010. 99th Dahlem conference on infection, inflammation and chronic inflammatory disorders: triggering of autoimmune diseases by infections. Clin Exp Immunol 160:15-21.

64. Doyle, H. A., and M. J. Mamula. 2005. Posttranslational modifications of selfantigens. Ann N Y Acad Sci 1050:1-9. 
65. Doyle, H. A., and M. J. Mamula. 2002. Posttranslational protein modifications: new flavors in the menu of autoantigens. Curr Opin Rheumatol 14:244-249.

66. Beltrami, A., M. Rossmann, M. T. Fiorillo, F. Paladini, R. Sorrentino, W. Saenger, et al. 2008. Citrullination-dependent differential presentation of a selfpeptide by HLA-B27 subtypes. J Biol Chem 283:27189-27199.

67. Doyle, H. A., and M. J. Mamula. 2001. Post-translational protein modifications in antigen recognition and autoimmunity. Trends Immunol 22:443-449.

68. Dieker, J., and S. Muller. 2009. Post-translational modifications, subcellular relocation and release in apoptotic microparticles: apoptosis turns nuclear proteins into autoantigens. Folia Histochem Cytobiol 47:343-348.

69. Dieker, J., B. Cisterna, F. Monneaux, M. Decossas, J. van der Vlag, M. Biggiogera, et al. 2008. Apoptosis-linked changes in the phosphorylation status and subcellular localization of the spliceosomal autoantigen U1-70K. Cell Death Differ 15:793-804.

70. Dieker, J. W., J. H. Fransen, C. C. van Bavel, J. P. Briand, C. W. Jacobs, S. Muller, et al. 2007. Apoptosis-induced acetylation of histones is pathogenic in systemic lupus erythematosus. Arthritis Rheum 56:1921-1933.

71. Corthay, A., J. Backlund, J. Broddefalk, E. Michaelsson, T. J. Goldschmidt, J. Kihlberg, et al. 1998. Epitope glycosylation plays a critical role for T cell recognition of type II collagen in collagen-induced arthritis. Eur J Immunol 28:2580-2590.

72. Myers, L. K., J. Myllyharju, M. Nokelainen, D. D. Brand, M. A. Cremer, J. M. Stuart, et al. 2004. Relevance of posttranslational modifications for the arthritogenicity of type II collagen. J Immunol 172:2970-2975.

73. Nijenhuis, S., A. J. Zendman, E. R. Vossenaar, G. J. Pruijn, and W. J. vanVenrooij. 2004. Autoantibodies to citrullinated proteins in rheumatoid arthritis: clinical performance and biochemical aspects of an RA-specific marker. Clin Chim Acta 350:17-34.

74. Zendman, A. J., E. R. Vossenaar, and W. J. van Venrooij. 2004. Autoantibodies to citrullinated (poly)peptides: a key diagnostic and prognostic marker for rheumatoid arthritis. Autoimmunity 37:295-299.

75. Hargraves, M. M., H. Richmond, and R. Morton. 1948. Presentation of two bone marrow elements; the tart cell and the L.E. cell. Mayo Clin Proc 23:25-28.

76. Haserick, J. R., and D. W. Bortz. 1949. Normal bone marrow inclusion phenomena induced by lupus erythematosus plasma. J Invest Dermatol 13:47-49.

77. Casciola-Rosen, L. A., G. Anhalt, and A. Rosen. 1994. Autoantigens targeted in systemic lupus erythematosus are clustered in two populations of surface structures on apoptotic keratinocytes. $J$ Exp Med 179:1317-1330.

78. Rosen, A., and L. Casciola-Rosen. 1999. Autoantigens as substrates for apoptotic proteases: implications for the pathogenesis of systemic autoimmune disease. Cell Death Differ 6:6-12.

79. Grodzicky, T., and K. B. Elkon. 2000. Apoptosis in rheumatic diseases. Am J Med 108:73-82.

80. Radic, M., T. Marion, and M. Monestier. 2004. Nucleosomes are exposed at the cell surface in apoptosis. J Immunol 172:6692-6700. 
81. Cocca, B. A., A. M. Cline, and M. Z. Radic. 2002. Blebs and apoptotic bodies are B cell autoantigens. J Immunol 169:159-166.

82. Lorenz, H. M., M. Grunke, T. Hieronymus, M. Herrmann, A. Kuhnel, B. Manger, et al. 1997. In vitro apoptosis and expression of apoptosis-related molecules in lymphocytes from patients with systemic lupus erythematosus and other autoimmune diseases. Arthritis Rheum 40:306-317.

83. Casciola-Rosen, L. A., G. J. Anhalt, and A. Rosen. 1995. DNA-dependent protein kinase is one of a subset of autoantigens specifically cleaved early during apoptosis. J Exp Med 182:1625-1634.

84. Utz, P. J., M. Hottelet, T. M. Le, S. J. Kim, M. E. Geiger, W. J. van Venrooij, et al. 1998. The 72-kDa component of signal recognition particle is cleaved during apoptosis. J Biol Chem 273:35362-35370.

85. Degen, W. G., G. J. Pruijn, J. M. Raats, and W. J. van Venrooij. 2000. Caspasedependent cleavage of nucleic acids. Cell Death Differ 7:616-627.

86. Ayukawa, K., S. Taniguchi, J. Masumoto, S. Hashimoto, H. Sarvotham, A. Hara, et al. 2000. La autoantigen is cleaved in the COOH terminus and loses the nuclear localization signal during apoptosis. J Biol Chem 275:34465-34470.

87. Sebbagh, M., C. Renvoize, J. Hamelin, N. Riche, J. Bertoglio, and J. Breard. 2001. Caspase-3-mediated cleavage of ROCK I induces MLC phosphorylation and apoptotic membrane blebbing. Nat Cell Biol 3:346-352.

88. Coleman, M. L., E. A. Sahai, M. Yeo, M. Bosch, A. Dewar, and M. F. Olson. 2001. Membrane blebbing during apoptosis results from caspase-mediated activation of ROCK I. Nat Cell Biol 3:339-345.

89. Viorritto, I. C., N. P. Nikolov, and R. M. Siegel. 2007. Autoimmunity versus tolerance: can dying cells tip the balance? Clin Immunol 122:125-134.

90. Botto, M., C. Dell'Agnola, A. E. Bygrave, E. M. Thompson, H. T. Cook, F. Petry, et al. 1998. Homozygous C1q deficiency causes glomerulonephritis associated with multiple apoptotic bodies. Nat Genet 19:56-59.

91. Scott, R. S., E. J. McMahon, S. M. Pop, E. A. Reap, R. Caricchio, P. L. Cohen, et al. 2001. Phagocytosis and clearance of apoptotic cells is mediated by MER. Nature 411:207-211.

92. Cohen, P. L., R. Caricchio, V. Abraham, T. D. Camenisch, J. C. Jennette, R. A. Roubey, et al. 2002. Delayed apoptotic cell clearance and lupus-like autoimmunity in mice lacking the c-mer membrane tyrosine kinase. $J$ Exp Med 196:135-140.

93. Hanayama, R., M. Tanaka, K. Miyasaka, K. Aozasa, M. Koike, Y. Uchiyama, et al. 2004. Autoimmune disease and impaired uptake of apoptotic cells in MFG-E8deficient mice. Science 304:1147-1150.

94. Herrmann, M., R. E. Voll, O. M. Zoller, M. Hagenhofer, B. B. Ponner, and J. R. Kalden. 1998. Impaired phagocytosis of apoptotic cell material by monocytederived macrophages from patients with systemic lupus erythematosus. Arthritis Rheum 41:1241-1250.

95. Baumann, I., W. Kolowos, R. E. Voll, B. Manger, U. Gaipl, W. L. Neuhuber, et al. 2002. Impaired uptake of apoptotic cells into tingible body macrophages in germinal centers of patients with systemic lupus erythematosus. Arthritis Rheum 46:191-201. 
96. Pluddemann, A., C. Neyen, and S. Gordon. 2007. Macrophage scavenger receptors and host-derived ligands. Methods 43:207-217.

97. Freeman, G. J., J. M. Casasnovas, D. T. Umetsu, and R. H. DeKruyff. 2010. TIM genes: a family of cell surface phosphatidylserine receptors that regulate innate and adaptive immunity. Immunol Rev 235:172-189.

98. Hanayama, R., M. Tanaka, K. Miwa, A. Shinohara, A. Iwamatsu, and S. Nagata. 2002. Identification of a factor that links apoptotic cells to phagocytes. Nature 417:182-187.

99. Anderson, H. A., C. A. Maylock, J. A. Williams, C. P. Paweletz, H. Shu, and E. Shacter. 2003. Serum-derived protein S binds to phosphatidylserine and stimulates the phagocytosis of apoptotic cells. Nat Immunol 4:87-91.

100. Fadok, V. A., D. L. Bratton, D. M. Rose, A. Pearson, R. A. Ezekewitz, and P. M. Henson. 2000. A receptor for phosphatidylserine-specific clearance of apoptotic cells. Nature 405:85-90.

101. Chen, Q., P. R. Stone, L. M. McCowan, and L. W. Chamley. 2006. Phagocytosis of necrotic but not apoptotic trophoblasts induces endothelial cell activation. Hypertension 47:116-121.

102. Parnaik, R., M. C. Raff, and J. Scholes. 2000. Differences between the clearance of apoptotic cells by professional and non-professional phagocytes. Curr Biol 10:857-860.

103. Wood, W., M. Turmaine, R. Weber, V. Camp, R. A. Maki, S. R. McKercher, et al. 2000. Mesenchymal cells engulf and clear apoptotic footplate cells in macrophageless PU.1 null mouse embryos. Development 127:5245-5252.

104. Monks, J., D. Rosner, F. J. Geske, L. Lehman, L. Hanson, M. C. Neville, et al. 2005. Epithelial cells as phagocytes: apoptotic epithelial cells are engulfed by mammary alveolar epithelial cells and repress inflammatory mediator release. Cell Death Differ 12:107-114.

105. Cline, A. M., and M. Z. Radic. 2004. Apoptosis, subcellular particles, and autoimmunity. Clin Immunol 112:175-182.

106. Gao, Y., J. M. Herndon, H. Zhang, T. S. Griffith, and T. A. Ferguson. 1998. Antiinflammatory effects of CD95 ligand (FasL)-induced apoptosis. $J$ Exp Med 188:887-896.

107. Chen, W., M. E. Frank, W. Jin, and S. M. Wahl. 2001. TGF-beta released by apoptotic T cells contributes to an immunosuppressive milieu. Immunity 14:715725.

108. Meagher, L. C., J. S. Savill, A. Baker, R. W. Fuller, and C. Haslett. 1992. Phagocytosis of apoptotic neutrophils does not induce macrophage release of thromboxane B2. J Leukoc Biol 52:269-273.

109. Fadok, V. A., D. L. Bratton, A. Konowal, P. W. Freed, J. Y. Westcott, and P. M. Henson. 1998. Macrophages that have ingested apoptotic cells in vitro inhibit proinflammatory cytokine production through autocrine/paracrine mechanisms involving TGF-beta, PGE2, and PAF. J Clin Invest 101:890-898.

110. Huynh, M. L., V. A. Fadok, and P. M. Henson. 2002. Phosphatidylserinedependent ingestion of apoptotic cells promotes TGF-beta1 secretion and the resolution of inflammation. J Clin Invest 109:41-50. 
111. Rothlin, C. V., S. Ghosh, E. I. Zuniga, M. B. Oldstone, and G. Lemke. 2007. TAM receptors are pleiotropic inhibitors of the innate immune response. Cell 131:1124-1136.

112. Byrne, A., and D. J. Reen. 2002. Lipopolysaccharide induces rapid production of IL-10 by monocytes in the presence of apoptotic neutrophils. J Immunol 168:1968-1977.

113. Cvetanovic, M., and D. S. Ucker. 2004. Innate immune discrimination of apoptotic cells: repression of proinflammatory macrophage transcription is coupled directly to specific recognition. J Immunol 172:880-889.

114. Cvetanovic, M., J. E. Mitchell, V. Patel, B. S. Avner, Y. Su, P. T. van der Saag, et al. 2006. Specific recognition of apoptotic cells reveals a ubiquitous and unconventional innate immunity. $J$ Biol Chem 281:20055-20067.

115. Patel, V. A., A. Longacre, K. Hsiao, H. Fan, F. Meng, J. E. Mitchell, et al. 2006. Apoptotic cells, at all stages of the death process, trigger characteristic signaling events that are divergent from and dominant over those triggered by necrotic cells: implications for the delayed clearance model of autoimmunity. J Biol Chem 281:4663-4670.

116. Li, M., D. F. Carpio, Y. Zheng, P. Bruzzo, V. Singh, F. Ouaaz, et al. 2001. An essential role of the NF-kappa B/Toll-like receptor pathway in induction of inflammatory and tissue-repair gene expression by necrotic cells. $J$ Immunol 166:7128-7135.

117. Piccinini, A. M., and K. S. Midwood. 2010. DAMPening inflammation by modulating TLR signalling. Mediators Inflamm 2010.

118. Matzinger, P. 2002. The danger model: a renewed sense of self. Science 296:301305.

119. Swamy, M., C. Jamora, W. Havran, and A. Hayday. 2010. Epithelial decision makers: in search of the 'epimmunome'. Nat Immunol 11:656-665.

120. Yang, D., P. Tewary, G. de la Rosa, F. Wei, and J. J. Oppenheim. 2010. The alarmin functions of high-mobility group proteins. Biochim Biophys Acta 1799:157-163.

121. Foell, D., H. Wittkowski, and J. Roth. 2007. Mechanisms of disease: a 'DAMP' view of inflammatory arthritis. Nat Clin Pract Rheumatol 3:382-390.

122. Vinuesa, C. G., M. A. Linterman, C. C. Goodnow, and K. L. Randall. 2010. T cells and follicular dendritic cells in germinal center B-cell formation and selection. Immunol Rev 237:72-89.

123. Nutt, S. L., and D. M. Tarlinton. 2011. Germinal center B and follicular helper T cells: siblings, cousins or just good friends? Nat Immunol 12:472-477.

124. Pulendran, B., and R. Ahmed. 2006. Translating innate immunity into immunological memory: implications for vaccine development. Cell 124:849863.

125. Di Noia, J. M., and M. S. Neuberger. 2007. Molecular mechanisms of antibody somatic hypermutation. Annu Rev Biochem 76:1-22.

126. William, J., C. Euler, S. Christensen, and M. J. Shlomchik. 2002. Evolution of autoantibody responses via somatic hypermutation outside of germinal centers. Science 297:2066-2070. 
127. Blander, J. M., and R. Medzhitov. 2006. On regulation of phagosome maturation and antigen presentation. Nat Immunol 7:1029-1035.

128. Blander, J. M., and R. Medzhitov. 2006. Toll-dependent selection of microbial antigens for presentation by dendritic cells. Nature 440:808-812.

129. McHeyzer-Williams, L. J., N. Pelletier, L. Mark, N. Fazilleau, and M. G. McHeyzer-Williams. 2009. Follicular helper T cells as cognate regulators of B cell immunity. Curr Opin Immunol 21:266-273.

130. Balazs, M., F. Martin, T. Zhou, and J. Kearney. 2002. Blood dendritic cells interact with splenic marginal zone B cells to initiate T-independent immune responses. Immunity 17:341-352.

131. MacLennan, I., and C. Vinuesa. 2002. Dendritic cells, BAFF, and APRIL: innate players in adaptive antibody responses. Immunity 17:235-238.

132. Peng, Y., R. Kowalewski, S. Kim, and K. B. Elkon. 2005. The role of IgM antibodies in the recognition and clearance of apoptotic cells. Mol Immunol 42:781-787.

133. Albert, M. L., S. F. Pearce, L. M. Francisco, B. Sauter, P. Roy, R. L. Silverstein, et al. 1998. Immature dendritic cells phagocytose apoptotic cells via alphavbeta5 and CD36, and cross-present antigens to cytotoxic T lymphocytes. $J$ Exp Med 188:1359-1368.

134. Belz, G. T., F. R. Carbone, and W. R. Heath. 2002. Cross-presentation of antigens by dendritic cells. Crit Rev Immunol 22:439-448.

135. Merad, M., and M. G. Manz. 2009. Dendritic cell homeostasis. Blood 113:34183427.

136. Cella, M., A. Engering, V. Pinet, J. Pieters, and A. Lanzavecchia. 1997. Inflammatory stimuli induce accumulation of MHC class II complexes on dendritic cells. Nature 388:782-787.

137. Sallusto, F., B. Palermo, D. Lenig, M. Miettinen, S. Matikainen, I. Julkunen, et al. 1999. Distinct patterns and kinetics of chemokine production regulate dendritic cell function. Eur J Immunol 29:1617-1625.

138. Langenkamp, A., M. Messi, A. Lanzavecchia, and F. Sallusto. 2000. Kinetics of dendritic cell activation: impact on priming of TH1, TH2 and nonpolarized T cells. Nat Immunol 1:311-316.

139. Granucci, F., C. Vizzardelli, N. Pavelka, S. Feau, M. Persico, E. Virzi, et al. 2001. Inducible IL-2 production by dendritic cells revealed by global gene expression analysis. Nat Immunol 2:882-888.

140. Inaba, K., S. Turley, T. Iyoda, F. Yamaide, S. Shimoyama, C. Reis e Sousa, et al. 2000. The formation of immunogenic major histocompatibility complex class IIpeptide ligands in lysosomal compartments of dendritic cells is regulated by inflammatory stimuli. $J$ Exp Med 191:927-936.

141. Kurts, C., H. Kosaka, F. R. Carbone, J. F. Miller, and W. R. Heath. 1997. Class Irestricted cross-presentation of exogenous self-antigens leads to deletion of autoreactive CD8(+) T cells. J Exp Med 186:239-245.

142. Adler, A. J., D. W. Marsh, G. S. Yochum, J. L. Guzzo, A. Nigam, W. G. Nelson, et al. 1998. CD4+ T cell tolerance to parenchymal self-antigens requires presentation by bone marrow-derived antigen-presenting cells. J Exp Med 187:1555-1564. 
143. Probst, H. C., J. Lagnel, G. Kollias, and M. van den Broek. 2003. Inducible transgenic mice reveal resting dendritic cells as potent inducers of CD8+ T cell tolerance. Immunity 18:713-720.

144. Hamilton-Williams, E. E., A. Lang, D. Benke, G. M. Davey, K. H. Wiesmuller, and C. Kurts. 2005. Cutting edge: TLR ligands are not sufficient to break crosstolerance to self-antigens. J Immunol 174:1159-1163.

145. Bekeredjian-Ding, I., and G. Jego. 2009. Toll-like receptors--sentries in the B-cell response. Immunology 128:311-323.

146. Genestier, L., M. Taillardet, P. Mondiere, H. Gheit, C. Bella, and T. Defrance. 2007. TLR agonists selectively promote terminal plasma cell differentiation of $B$ cell subsets specialized in thymus-independent responses. J Immunol 178:77797786.

147. Rifkin, I. R., E. A. Leadbetter, L. Busconi, G. Viglianti, and A. MarshakRothstein. 2005. Toll-like receptors, endogenous ligands, and systemic autoimmune disease. Immunol Rev 204:27-42.

148. Leadbetter, E. A., I. R. Rifkin, A. M. Hohlbaum, B. C. Beaudette, M. J. Shlomchik, and A. Marshak-Rothstein. 2002. Chromatin-IgG complexes activate B cells by dual engagement of IgM and Toll-like receptors. Nature 416:603-607.

149. Papadimitraki, E. D., G. K. Bertsias, and D. T. Boumpas. 2007. Toll like receptors and autoimmunity: a critical appraisal. J Autoimmun 29:310-318.

150. Cunningham, M. W., J. M. McCormack, L. R. Talaber, J. B. Harley, E. M. Ayoub, R. S. Muneer, et al. 1988. Human monoclonal antibodies reactive with antigens of the group A Streptococcus and human heart. J Immunol 141:27602766 .

151. Ellis, N. M., Y. Li, W. Hildebrand, V. A. Fischetti, and M. W. Cunningham. 2005. T cell mimicry and epitope specificity of cross-reactive $\mathrm{T}$ cell clones from rheumatic heart disease. J Immunol 175:5448-5456.

152. Gauntt, C. J., H. M. Arizpe, A. L. Higdon, H. J. Wood, D. F. Bowers, M. M. Rozek, et al. 1995. Molecular mimicry, anti-coxsackievirus B3 neutralizing monoclonal antibodies, and myocarditis. J Immunol 154:2983-2995.

153. Gauntt, C. J., A. L. Higdon, H. M. Arizpe, M. R. Tamayo, R. Crawley, R. D. Henkel, et al. 1993. Epitopes shared between coxsackievirus B3 (CVB3) and normal heart tissue contribute to CVB3-induced murine myocarditis. Clin Immunol Immunopathol 68:129-134.

154. Aspinall, G. O., S. Fujimoto, A. G. McDonald, H. Pang, L. A. Kurjanczyk, and J. L. Penner. 1994. Lipopolysaccharides from Campylobacter jejuni associated with Guillain-Barre syndrome patients mimic human gangliosides in structure. Infect Immun 62:2122-2125.

155. Yuki, N., T. Taki, M. Takahashi, K. Saito, T. Tai, T. Miyatake, et al. 1994. Penner's serotype 4 of Campylobacter jejuni has a lipopolysaccharide that bears a GM1 ganglioside epitope as well as one that bears a GD1 a epitope. Infect Immun 62:2101-2103.

156. Yuki, N., K. Susuki, M. Koga, Y. Nishimoto, M. Odaka, K. Hirata, et al. 2004. Carbohydrate mimicry between human ganglioside GM1 and Campylobacter jejuni lipooligosaccharide causes Guillain-Barre syndrome. Proc Natl Acad Sci U $S$ A 101:11404-11409. 
157. Steinman, R. M., D. Hawiger, and M. C. Nussenzweig. 2003. Tolerogenic dendritic cells. Anпu Rev Immunol 21:685-711.

158. Unutmaz, D., P. Pileri, and S. Abrignani. 1994. Antigen-independent activation of naive and memory resting $\mathrm{T}$ cells by a cytokine combination. $J$ Exp Med 180:1159-1164.

159. Ehl, S., J. Hombach, P. Aichele, H. Hengartner, and R. M. Zinkernagel. 1997. Bystander activation of cytotoxic T cells: studies on the mechanism and evaluation of in vivo significance in a transgenic mouse model. J Exp Med 185:1241-1251.

160. Kaprio, J., J. Tuomilehto, M. Koskenvuo, K. Romanov, A. Reunanen, J. Eriksson, et al. 1992. Concordance for type 1 (insulin-dependent) and type 2 (non-insulindependent) diabetes mellitus in a population-based cohort of twins in Finland. Diabetologia 35:1060-1067.

161. Kyvik, K. O., A. Green, and H. Beck-Nielsen. 1995. Concordance rates of insulin dependent diabetes mellitus: a population based study of young Danish twins. BMJ 311:913-917.

162. Islam, T., W. J. Gauderman, W. Cozen, A. S. Hamilton, M. E. Burnett, and T. M. Mack. 2006. Differential twin concordance for multiple sclerosis by latitude of birthplace. Ann Neurol 60:56-64.

163. Ginsberg-Fellner, F., M. E. Witt, S. Yagihashi, M. J. Dobersen, F. Taub, B. Fedun, et al. 1984. Congenital rubella syndrome as a model for type 1 (insulindependent) diabetes mellitus: increased prevalence of islet cell surface antibodies. Diabetologia 27 Suppl:87-89.

164. Pak, C. Y., C. Y. Cha, R. V. Rajotte, R. G. McArthur, and J. W. Yoon. 1990. Human pancreatic islet cell specific 38 kilodalton autoantigen identified by cytomegalovirus-induced monoclonal islet cell autoantibody. Diabetologia 33:569-572.

165. Serreze, D. V., E. W. Ottendorfer, T. M. Ellis, C. J. Gauntt, and M. A. Atkinson. 2000. Acceleration of type 1 diabetes by a coxsackievirus infection requires a preexisting critical mass of autoreactive T-cells in pancreatic islets. Diabetes 49:708-711.

166. Miller, S. D., C. L. Vanderlugt, W. S. Begolka, W. Pao, R. L. Yauch, K. L. Neville, et al. 1997. Persistent infection with Theiler's virus leads to CNS autoimmunity via epitope spreading. Nat Med 3:1133-1136.

167. Akirav, E. M., N. H. Ruddle, and K. C. Herold. 2011. The role of AIRE in human autoimmune disease. Nat Rev Endocrinol 7:25-33.

168. Markovic-Plese, S., H. Fukaura, J. Zhang, A. al-Sabbagh, S. Southwood, A. Sette, et al. 1995. T cell recognition of immunodominant and cryptic proteolipid protein epitopes in humans. J Immunol 155:982-992.

169. Matsuo, H., A. P. Batocchi, S. Hawke, M. Nicolle, L. Jacobson, A. Vincent, et al. 1995. Peptide-selected T cell lines from myasthenia gravis patients and controls recognize epitopes that are not processed from whole acetylcholine receptor. $J$ Immunol 155:3683-3692. 
170. Redmond, T. M., H. Sanui, L. H. Hu, B. Wiggert, H. Margalit, J. A. Berzofsky, et al. 1989. Immune responses to peptides derived from the retinal protein IRBP: immunopathogenic determinants are not necessarily immunodominant. Clin Immunol Immunopathol 53:212-224.

171. Hu, L. H., T. M. Redmond, H. Sanui, T. Kuwabara, C. G. McAllister, B. Wiggert, et al. 1989. Rat T-cell lines specific to a nonimmunodominant determinant of a retinal protein (IRBP) produce uveoretinitis and pinealitis. Cell Immunol 122:251261.

172. Inomata, T., H. Hanawa, T. Miyanishi, E. Yajima, S. Nakayama, T. Maita, et al. 1995. Localization of porcine cardiac myosin epitopes that induce experimental autoimmune myocarditis. Circ Res 76:726-733.

173. Bockenstedt, L. K., R. J. Gee, and M. J. Mamula. 1995. Self-peptides in the initiation of lupus autoimmunity. J Immunol 154:3516-3524.

174. Tuohy, V. K., and D. M. Thomas. 1995. Sequence 104-117 of myelin proteolipid protein is a cryptic encephalitogenic T cell determinant for $\mathrm{SJL} / \mathrm{J}$ mice. $J$ Neuroimmunol 56:161-170.

175. Korkmaz, B., M. S. Horwitz, D. E. Jenne, and F. Gauthier. 2010. Neutrophil elastase, proteinase 3, and cathepsin $\mathrm{G}$ as therapeutic targets in human diseases. Pharmacol Rev 62:726-759.

176. Arnhold, J., and J. Flemmig. 2010. Human myeloperoxidase in innate and acquired immunity. Arch Biochem Biophys 500:92-106.

177. Watorek, W. 2003. Azurocidin -- inactive serine proteinase homolog acting as a multifunctional inflammatory mediator. Acta Biochim Pol 50:743-752.

178. Soehnlein, O., and L. Lindbom. 2009. Neutrophil-derived azurocidin alarms the immune system. J Leukoc Biol 85:344-351.

179. Klebanoff, S. J. 2005. Myeloperoxidase: friend and foe. J Leukoc Biol 77:598625.

180. Good, R. A., P. G. Quie, D. B. Windhorst, A. R. Page, G. E. Rodey, J. White, et al. 1968. Fatal (chronic) granulomatous disease of childhood: a hereditary defect of leukocyte function. Semin Hematol 5:215-254.

181. Weiss, J., P. Elsbach, I. Olsson, and H. Odeberg. 1978. Purification and characterization of a potent bactericidal and membrane active protein from the granules of human polymorphonuclear leukocytes. J Biol Chem 253:2664-2672.

182. Bennouna, S., S. K. Bliss, T. J. Curiel, and E. Y. Denkers. 2003. Cross-talk in the innate immune system: neutrophils instruct recruitment and activation of dendritic cells during microbial infection. J Immunol 171:6052-6058.

183. Chertov, O., H. Ueda, L. L. Xu, K. Tani, W. J. Murphy, J. M. Wang, et al. 1997. Identification of human neutrophil-derived cathepsin G and azurocidin/CAP37 as chemoattractants for mononuclear cells and neutrophils. J Exp Med 186:739-747.

184. Wittamer, V., B. Bondue, A. Guillabert, G. Vassart, M. Parmentier, and D. Communi. 2005. Neutrophil-mediated maturation of chemerin: a link between innate and adaptive immunity. J Immunol 175:487-493.

185. van Gisbergen, K. P., M. Sanchez-Hernandez, T. B. Geijtenbeek, and Y. van Kooyk. 2005. Neutrophils mediate immune modulation of dendritic cells through glycosylation-dependent interactions between Mac-1 and DC-SIGN. J Exp Med 201:1281-1292. 
186. Scapini, P., A. Carletto, B. Nardelli, F. Calzetti, V. Roschke, F. Merigo, et al. 2005. Proinflammatory mediators elicit secretion of the intracellular Blymphocyte stimulator pool (BLyS) that is stored in activated neutrophils: implications for inflammatory diseases. Blood 105:830-837.

187. Ethuin, F., B. Gerard, J. E. Benna, A. Boutten, M. A. Gougereot-Pocidalo, L. Jacob, et al. 2004. Human neutrophils produce interferon gamma upon stimulation by interleukin-12. Lab Invest 84:1363-1371.

188. Gosselin, E. J., K. Wardwell, W. F. Rigby, and P. M. Guyre. 1993. Induction of MHC class II on human polymorphonuclear neutrophils by granulocyte/macrophage colony-stimulating factor, IFN-gamma, and IL-3. $J$ Immunol 151:1482-1490.

189. Smith, W. B., L. Guida, Q. Sun, E. I. Korpelainen, C. van den Heuvel, D. Gillis, et al. 1995. Neutrophils activated by granulocyte-macrophage colony-stimulating factor express receptors for interleukin-3 which mediate class II expression. Blood 86:3938-3944.

190. Radsak, M., C. Iking-Konert, S. Stegmaier, K. Andrassy, and G. M. Hansch. 2000. Polymorphonuclear neutrophils as accessory cells for T-cell activation: major histocompatibility complex class II restricted antigen-dependent induction of T-cell proliferation. Immunology 101:521-530.

191. Hansch, G. M., M. Radsak, C. Wagner, B. Reis, A. Koch, A. Breitbart, et al. 1999. Expression of major histocompatibility class II antigens on polymorphonuclear neutrophils in patients with Wegener's granulomatosis. Kidney Int 55:1811-1818.

192. Iking-Konert, C., S. Vogt, M. Radsak, C. Wagner, G. M. Hansch, and K. Andrassy. 2001. Polymorphonuclear neutrophils in Wegener's granulomatosis acquire characteristics of antigen presenting cells. Kidney Int 60:2247-2262.

193. Iking-Konert, C., B. Ostendorf, O. Sander, M. Jost, C. Wagner, L. Joosten, et al. 2005. Transdifferentiation of polymorphonuclear neutrophils to dendritic-like cells at the site of inflammation in rheumatoid arthritis: evidence for activation by T cells. Ann Rheum Dis 64:1436-1442.

194. Brinkmann, V., U. Reichard, C. Goosmann, B. Fauler, Y. Uhlemann, D. S. Weiss, et al. 2004. Neutrophil extracellular traps kill bacteria. Science 303:1532-1535.

195. Urban, C. F., U. Reichard, V. Brinkmann, and A. Zychlinsky. 2006. Neutrophil extracellular traps capture and kill Candida albicans yeast and hyphal forms. Cell Microbiol 8:668-676.

196. Fuchs, T. A., U. Abed, C. Goosmann, R. Hurwitz, I. Schulze, V. Wahn, et al. 2007. Novel cell death program leads to neutrophil extracellular traps. J Cell Biol 176:231-241.

197. Neeli, I., N. Dwivedi, S. Khan, and M. Radic. 2009. Regulation of extracellular chromatin release from neutrophils. J Innate Immun 1:194-201.

198. Bianchi, M., A. Hakkim, V. Brinkmann, U. Siler, R. A. Seger, A. Zychlinsky, et al. 2009. Restoration of NET formation by gene therapy in CGD controls aspergillosis. Blood 114:2619-2622.

199. Lim, M. B., J. W. Kuiper, A. Katchky, H. Goldberg, and M. Glogauer. 2011. Rac2 is required for the formation of neutrophil extracellular traps. J Leukoc Biol (In Press). 
200. Metzler, K. D., T. A. Fuchs, W. M. Nauseef, D. Reumaux, J. Roesler, I. Schulze, et al. 2011. Myeloperoxidase is required for neutrophil extracellular trap formation: implications for innate immunity. Blood 117:953-959.

201. Papayannopoulos, V., K. D. Metzler, A. Hakkim, and A. Zychlinsky. 2010. Neutrophil elastase and myeloperoxidase regulate the formation of neutrophil extracellular traps. J Cell Biol 191:677-691.

202. Neeli, I., S. N. Khan, and M. Radic. 2008. Histone deimination as a response to inflammatory stimuli in neutrophils. J Immunol 180:1895-1902.

203. Wang, Y., M. Li, S. Stadler, S. Correll, P. Li, D. Wang, et al. 2009. Histone hypercitrullination mediates chromatin decondensation and neutrophil extracellular trap formation. J Cell Biol 184:205-213.

204. Chavanas, S., M. C. Mechin, H. Takahara, A. Kawada, R. Nachat, G. Serre, et al. 2004. Comparative analysis of the mouse and human peptidylarginine deiminase gene clusters reveals highly conserved non-coding segments and a new human gene, PADI6. Gene 330:19-27.

205. Vossenaar, E. R., A. J. Zendman, W. J. van Venrooij, and G. J. Pruijn. 2003. PAD, a growing family of citrullinating enzymes: genes, features and involvement in disease. Bioessays 25:1106-1118.

206. Knuckley, B., M. Bhatia, and P. R. Thompson. 2007. Protein arginine deiminase 4: evidence for a reverse protonation mechanism. Biochemistry 46:6578-6587.

207. Ke, Z., Y. Zhou, P. Hu, S. Wang, D. Xie, and Y. Zhang. 2009. Active site cysteine is protonated in the PAD4 Michaelis complex: evidence from BornOppenheimer ab initio QM/MM molecular dynamics simulations. J Phys Chem B 113:12750-12758.

208. Tarcsa, E., L. N. Marekov, G. Mei, G. Melino, S. C. Lee, and P. M. Steinert. 1996. Protein unfolding by peptidylarginine deiminase. Substrate specificity and structural relationships of the natural substrates trichohyalin and filaggrin. $J$ Biol Chem 271:30709-30716.

209. Senshu, T., K. Akiyama, S. Kan, H. Asaga, A. Ishigami, and M. Manabe. 1995. Detection of deiminated proteins in rat skin: probing with a monospecific antibody after modification of citrulline residues. J Invest Dermatol 105:163-169.

210. Senshu, T., S. Kan, H. Ogawa, M. Manabe, and H. Asaga. 1996. Preferential deimination of keratin $\mathrm{K} 1$ and filaggrin during the terminal differentiation of human epidermis. Biochem Biophys Res Commun 225:712-719.

211. Gyorgy, B., E. Toth, E. Tarcsa, A. Falus, and E. I. Buzas. 2006. Citrullination: a posttranslational modification in health and disease. Int J Biochem Cell Biol 38:1662-1677.

212. Moscarello, M. A., D. D. Wood, C. Ackerley, and C. Boulias. 1994. Myelin in multiple sclerosis is developmentally immature. J Clin Invest 94:146-154.

213. Li, P., M. Li, M. R. Lindberg, M. J. Kennett, N. Xiong, and Y. Wang. 2010. PAD4 is essential for antibacterial innate immunity mediated by neutrophil extracellular traps. $J$ Exp Med 207:1853-1862.

214. Girbal-Neuhauser, E., J. J. Durieux, M. Arnaud, P. Dalbon, M. Sebbag, C. Vincent, et al. 1999. The epitopes targeted by the rheumatoid arthritis-associated antifilaggrin autoantibodies are posttranslationally generated on various sites of (pro)filaggrin by deimination of arginine residues. J Immunol 162:585-594. 
215. Van Steendam, K., K. Tilleman, M. De Ceuleneer, F. De Keyser, D. Elewaut, and D. Deforce. 2010. Citrullinated vimentin as an important antigen in immune complexes from synovial fluid of rheumatoid arthritis patients with antibodies against citrullinated proteins. Arthritis Res Ther 12:R132.

216. Tabushi, Y., T. Nakanishi, T. Takeuchi, M. Nakajima, K. Ueda, T. Kotani, et al. 2008. Detection of citrullinated proteins in synovial fluids derived from patients with rheumatoid arthritis by proteomics-based analysis. Ann Clin Biochem 45:413-417.

217. Schellekens, G. A., H. Visser, B. A. de Jong, F. H. van den Hoogen, J. M. Hazes, F. C. Breedveld, et al. 2000. The diagnostic properties of rheumatoid arthritis antibodies recognizing a cyclic citrullinated peptide. Arthritis Rheum 43:155-163.

218. Bang, H., K. Egerer, A. Gauliard, K. Luthke, P. E. Rudolph, G. Fredenhagen, et al. 2007. Mutation and citrullination modifies vimentin to a novel autoantigen for rheumatoid arthritis. Arthritis Rheum 56:2503-2511.

219. Luime, J. J., E. M. Colin, J. M. Hazes, and E. Lubberts. 2010. Does anti-mutated citrullinated vimentin have additional value as a serological marker in the diagnostic and prognostic investigation of patients with rheumatoid arthritis? A systematic review. Ann Rheum Dis 69:337-344.

220. Rantapaa-Dahlqvist, S., B. A. de Jong, E. Berglin, G. Hallmans, G. Wadell, H. Stenlund, et al. 2003. Antibodies against cyclic citrullinated peptide and IgA rheumatoid factor predict the development of rheumatoid arthritis. Arthritis Rheum 48:2741-2749.

221. van Gaalen, F. A., S. P. Linn-Rasker, W. J. van Venrooij, B. A. de Jong, F. C. Breedveld, C. L. Verweij, et al. 2004. Autoantibodies to cyclic citrullinated peptides predict progression to rheumatoid arthritis in patients with undifferentiated arthritis: a prospective cohort study. Arthritis Rheum 50:709-715.

222. Tranquill, L. R., L. Cao, N. C. Ling, H. Kalbacher, R. M. Martin, and J. N. Whitaker. 2000. Enhanced T cell responsiveness to citrulline-containing myelin basic protein in multiple sclerosis patients. Mult Scler 6:220-225.

223. Cao, L., D. Sun, and J. N. Whitaker. 1998. Citrullinated myelin basic protein induces experimental autoimmune encephalomyelitis in Lewis rats through a diverse T cell repertoire. J Neuroimmunol 88:21-29.

224. Hill, J. A., S. Southwood, A. Sette, A. M. Jevnikar, D. A. Bell, and E. Cairns. 2003. Cutting edge: the conversion of arginine to citrulline allows for a highaffinity peptide interaction with the rheumatoid arthritis-associated HLADRB1*0401 MHC class II molecule. J Immunol 171:538-541.

225. Gregersen, P. K., J. Silver, and R. J. Winchester. 1987. The shared epitope hypothesis. An approach to understanding the molecular genetics of susceptibility to rheumatoid arthritis. Arthritis Rheum 30:1205-1213.

226. Gorman, J. D., and L. A. Criswell. 2002. The shared epitope and severity of rheumatoid arthritis. Rheum Dis Clin North Am 28:59-78.

227. Plenge, R. M. 2009. Rheumatoid arthritis genetics: 2009 update. Curr Rheumatol Rep 11:351-356. 
228. Suzuki, A., R. Yamada, X. Chang, S. Tokuhiro, T. Sawada, M. Suzuki, et al. 2003. Functional haplotypes of PADI4, encoding citrullinating enzyme peptidylarginine deiminase 4, are associated with rheumatoid arthritis. Nat Genet 34:395-402.

229. Zhao, J., Y. Zhao, J. He, R. Jia, and Z. Li. 2008. Prevalence and significance of anti-peptidylarginine deiminase 4 antibodies in rheumatoid arthritis. J Rheumatol 35:969-974.

230. Chang, X., R. Yamada, A. Suzuki, Y. Kochi, T. Sawada, and K. Yamamoto. 2005. Citrullination of fibronectin in rheumatoid arthritis synovial tissue. Rheumatology (Oxford) 44:1374-1382.

231. Takizawa, Y., A. Suzuki, T. Sawada, M. Ohsaka, T. Inoue, R. Yamada, et al. 2006. Citrullinated fibrinogen detected as a soluble citrullinated autoantigen in rheumatoid arthritis synovial fluids. Ann Rheum Dis 65:1013-1020.

232. Halvorsen, E. H., S. Pollmann, I. M. Gilboe, D. van der Heijde, R. Landewe, S. Odegard, et al. 2008. Serum IgG antibodies to peptidylarginine deiminase 4 in rheumatoid arthritis and associations with disease severity. Ann Rheum Dis 67:414-417.

233. Oehmcke, S., M. Morgelin, and H. Herwald. 2009. Activation of the human contact system on neutrophil extracellular traps. J Innate Immun 1:225-230.

234. Joseph, K., and A. P. Kaplan. 2005. Formation of bradykinin: a major contributor to the innate inflammatory response. Adv Immunol 86:159-208.

235. Manolova, I., M. Dancheva, and K. Halacheva. 2001. Antineutrophil cytoplasmic antibodies in patients with systemic lupus erythematosus: prevalence, antigen specificity, and clinical associations. Rheumatol Int 20:197-204.

236. Pradhan, V. D., S. S. Badakere, L. S. Bichile, and A. F. Almeida. 2004. Antineutrophil cytoplasmic antibodies (ANCA) in systemic lupus erythematosus: prevalence, clinical associations and correlation with other autoantibodies. $J$ Assoc Physicians India 52:533-537.

237. Lovell, D. J., and N. M. Ruth. 2005. Pediatric clinical research. Curr Opin Rheumatol 17:265-270.

238. Tamiya, H., K. Tani, J. Miyata, K. Sato, T. Urata, B. Lkhagvaa, et al. 2006. Defensins- and cathepsin G-ANCA in systemic lupus erythematosus. Rheumatol Int 27:147-152.

239. Froy, O., and Z. M. Sthoeger. 2009. Defensins in systemic lupus erythematosus. Ann N Y Acad Sci 1173:365-369.

240. Lande, R., D. Ganguly, V. Facchinetti, L. Frasca, C. Conrad, J. Gregorio, et al. 2011. Neutrophils activate plasmacytoid dendritic cells by releasing self-DNApeptide complexes in systemic lupus erythematosus. Sci Transl Med 3:73ra19.

241. Garcia-Romo, G. S., S. Caielli, B. Vega, J. Connolly, F. Allantaz, Z. Xu, et al. 2011. Netting neutrophils are major inducers of type I IFN production in pediatric systemic lupus erythematosus. Sci Transl Med 3:73ra20.

242. Villanueva, E., S. Yalavarthi, C. C. Berthier, J. B. Hodgin, R. Khandpur, A. M. Lin, et al. 2011. Netting neutrophils induce endothelial damage, infiltrate tissues, and expose immunostimulatory molecules in systemic lupus erythematosus. $J$ Immunol 187:538-552. 
243. Hakkim, A., B. G. Furnrohr, K. Amann, B. Laube, U. A. Abed, V. Brinkmann, et al. 2010. Impairment of neutrophil extracellular trap degradation is associated with lupus nephritis. Proc Natl Acad Sci U S A 107:9813-9818.

244. Urban, C. F., D. Ermert, M. Schmid, U. Abu-Abed, C. Goosmann, W. Nacken, et al. 2009. Neutrophil extracellular traps contain calprotectin, a cytosolic protein complex involved in host defense against Candida albicans. PLoS Pathog 5:e1000639.

245. Suzuki, T., R. W. Burlingame, C. A. Casiano, M. L. Boey, and R. L. Rubin. 1994. Antihistone antibodies in systemic lupus erythematosus: assay dependency and effects of ubiquitination and serum DNA. J Rheumatol 21:1081-1091.

246. Cohen, M. G., and J. Webb. 1989. Antihistone antibodies in rheumatoid arthritis and Felty's syndrome. Arthritis Rheum 32:1319-1324.

247. Huston, J. M., M. Rosas-Ballina, X. Xue, O. Dowling, K. Ochani, M. Ochani, et al. 2009. Cholinergic neural signals to the spleen down-regulate leukocyte trafficking via CD11b. J Immunol 183:552-559.

248. Manoury, B., E. W. Hewitt, N. Morrice, P. M. Dando, A. J. Barrett, and C. Watts. 1998. An asparaginyl endopeptidase processes a microbial antigen for class II MHC presentation. Nature 396:695-699.

249. Kominami, E., T. Tsukahara, Y. Bando, and N. Katunuma. 1985. Distribution of cathepsins B and $\mathrm{H}$ in rat tissues and peripheral blood cells. $J$ Biochem 98:87-93.

250. Brix, K., A. Dunkhorst, K. Mayer, and S. Jordans. 2008. Cysteine cathepsins: cellular roadmap to different functions. Biochimie 90:194-207.

251. Clements, C. S., L. Kjer-Nielsen, L. Kostenko, H. L. Hoare, M. A. Dunstone, E. Moses, et al. 2005. Crystal structure of HLA-G: a nonclassical MHC class I molecule expressed at the fetal-maternal interface. Proc Natl Acad Sci U S A 102:3360-3365.

252. Barr, S. G., A. Zonana-Nacach, L. S. Magder, and M. Petri. 1999. Patterns of disease activity in systemic lupus erythematosus. Arthritis Rheum 42:2682-2688.

253. Aletaha, D., F. C. Breedveld, and J. S. Smolen. 2005. The need for new classification criteria for rheumatoid arthritis. Arthritis Rheum 52:3333-3336.

254. Balint, G. P., and P. V. Balint. 2004. Felty's syndrome. Best Pract Res Clin Rheumatol 18:631-645.

255. Liu, X., and T. P. Loughran, Jr. 2011. The spectrum of large granular lymphocyte leukemia and Felty's syndrome. Curr Opin Hematol 18:254-259.

256. Rose, N. R. 1998. The role of infection in the pathogenesis of autoimmune disease. Semin Immunol 10:5-13.

257. Grassme, H., V. Jendrossek, and E. Gulbins. 2001. Molecular mechanisms of bacteria induced apoptosis. Apoptosis 6:441-445.

258. Nagata, S., R. Hanayama, and K. Kawane. 2010. Autoimmunity and the clearance of dead cells. Cell 140:619-630.

259. Munafo, D. B., J. L. Johnson, A. A. Brzezinska, B. A. Ellis, M. R. Wood, and S. D. Catz. 2009. DNase I inhibits a late phase of reactive oxygen species production in neutrophils. $J$ Innate Immun 1:527-542.

260. Schnabel, A., E. Csernok, D. A. Isenberg, C. Mrowka, and W. L. Gross. 1995. Antineutrophil cytoplasmic antibodies in systemic lupus erythematosus. Prevalence, specificities, and clinical significance. Arthritis Rheum 38:633-637. 
261. Nassberger, L., H. Jonsson, A. G. Sjoholm, G. Sturfelt, and A. Heubner. 1989. Circulating anti-elastase in systemic lupus erythematosus. Lancet 1:509.

262. Pritzker, L. B., S. Joshi, J. J. Gowan, G. Harauz, and M. A. Moscarello. 2000. Deimination of myelin basic protein. 1. Effect of deimination of arginyl residues of myelin basic protein on its structure and susceptibility to digestion by cathepsin D. Biochemistry 39:5374-5381.

263. Masson-Bessiere, C., M. Sebbag, E. Girbal-Neuhauser, L. Nogueira, C. Vincent, T. Senshu, et al. 2001. The major synovial targets of the rheumatoid arthritisspecific antifilaggrin autoantibodies are deiminated forms of the alpha- and betachains of fibrin. J Immunol 166:4177-4184.

264. Vossenaar, E. R., N. Despres, E. Lapointe, A. van der Heijden, M. Lora, T. Senshu, et al. 2004. Rheumatoid arthritis specific anti-Sa antibodies target citrullinated vimentin. Arthritis Res Ther 6:R142-150.

265. Kuhn, K. A., L. Kulik, B. Tomooka, K. J. Braschler, W. P. Arend, W. H. Robinson, et al. 2006. Antibodies against citrullinated proteins enhance tissue injury in experimental autoimmune arthritis. J Clin Invest 116:961-973.

266. Uysal, H., R. Bockermann, K. S. Nandakumar, B. Sehnert, E. Bajtner, A. Engstrom, et al. 2009. Structure and pathogenicity of antibodies specific for citrullinated collagen type II in experimental arthritis. J Exp Med 206:449-462.

267. Schellekens, G. A., B. A. de Jong, F. H. van den Hoogen, L. B. van de Putte, and W. J. van Venrooij. 1998. Citrulline is an essential constituent of antigenic determinants recognized by rheumatoid arthritis-specific autoantibodies. J Clin Invest 101:273-281.

268. Nakashima, K., T. Hagiwara, and M. Yamada. 2002. Nuclear localization of peptidylarginine deiminase $\mathrm{V}$ and histone deimination in granulocytes. $J$ Biol Chem 277:49562-49568.

269. Tuaillon, N., S. Muller, J. L. Pasquali, P. Bordigoni, P. Youinou, and M. H. Van Regenmortel. 1990. Antibodies from patients with rheumatoid arthritis and juvenile chronic arthritis analyzed with core histone synthetic peptides. Int Arch Allergy Appl Immunol 91:297-305.

270. Miriovsky, B. J., K. Michaud, G. M. Thiele, J. R. O'Dell, G. W. Cannon, G. Kerr, et al. 2010. Anti-CCP antibody and rheumatoid factor concentrations predict greater disease activity in men with rheumatoid arthritis. Ann Rheum Dis 69:12921297.

271. Arnett, F. C., S. M. Edworthy, D. A. Bloch, D. J. McShane, J. F. Fries, N. S. Cooper, et al. 1988. The American Rheumatism Association 1987 revised criteria for the classification of rheumatoid arthritis. Arthritis Rheum 31:315-324.

272. Tan, E. M., A. S. Cohen, J. F. Fries, A. T. Masi, D. J. McShane, N. F. Rothfield, et al. 1982. The 1982 revised criteria for the classification of systemic lupus erythematosus. Arthritis Rheum 25:1271-1277.

273. Jennette, J. C., R. J. Falk, K. Andrassy, P. A. Bacon, J. Churg, W. L. Gross, et al. 1994. Nomenclature of systemic vasculitides. Proposal of an international consensus conference. Arthritis Rheum 37:187-192. 
274. Kearney, P. L., M. Bhatia, N. G. Jones, L. Yuan, M. C. Glascock, K. L. Catchings, et al. 2005. Kinetic characterization of protein arginine deiminase 4: a transcriptional corepressor implicated in the onset and progression of rheumatoid arthritis. Biochemistry 44:10570-10582.

275. Csernok, E., J. Holle, B. Hellmich, J. Willem, C. Tervaert, C. G. Kallenberg, et al. 2004. Evaluation of capture ELISA for detection of antineutrophil cytoplasmic antibodies directed against proteinase 3 in Wegener's granulomatosis: first results from a multicentre study. Rheumatology (Oxford) 43:174-180.

276. Mathsson, L., M. Mullazehi, M. C. Wick, O. Sjoberg, R. van Vollenhoven, L. Klareskog, et al. 2008. Antibodies against citrullinated vimentin in rheumatoid arthritis: higher sensitivity and extended prognostic value concerning future radiographic progression as compared with antibodies against cyclic citrullinated peptides. Arthritis Rheum 58:36-45.

277. Losman, M. J., T. M. Fasy, K. E. Novick, and M. Monestier. 1993. Relationships among antinuclear antibodies from autoimmune MRL mice reacting with histone H2A-H2B dimers and DNA. Int Immunol 5:513-523.

278. Csernok, E. 2003. Anti-neutrophil cytoplasmic antibodies and pathogenesis of small vessel vasculitides. Autoimmun Rev 2:158-164.

279. Jenuwein, T., and C. D. Allis. 2001. Translating the histone code. Science 293:1074-1080.

280. Casciola-Rosen, L., F. Andrade, D. Ulanet, W. B. Wong, and A. Rosen. 1999. Cleavage by granzyme B is strongly predictive of autoantigen status: implications for initiation of autoimmunity. J Exp Med 190:815-826.

281. Utz, P. J., T. J. Gensler, and P. Anderson. 2000. Death, autoantigen modifications, and tolerance. Arthritis Res 2:101-114.

282. Dieker, J., and S. Muller. 2010. Epigenetic histone code and autoimmunity. Clin Rev Allergy Immunol 39:78-84.

283. Andrade, F., E. Darrah, M. Gucek, R. N. Cole, A. Rosen, and X. Zhu. 2010. Autocitrullination of human peptidyl arginine deiminase type 4 regulates protein citrullination during cell activation. Arthritis Rheum 62:1630-1640.

284. Kolfenbach, J. R., K. D. Deane, L. A. Derber, C. I. O'Donnell, W. R. Gilliland, J. D. Edison, et al. 2010. Autoimmunity to peptidyl arginine deiminase type 4 precedes clinical onset of rheumatoid arthritis. Arthritis Rheum 62:2633-2639.

285. Brinkmann, V., and A. Zychlinsky. 2007. Beneficial suicide: why neutrophils die to make NETs. Nat Rev Microbiol 5:577-582.

286. Dancey, J. T., and L. H. Brubaker. 1979. Neutrophil marrow profiles in patients with rheumatoid arthritis and neutropenia. Br J Haematol 43:607-617.

287. Holle, J. U., Q. J. Wu, F. Moosig, W. L. Gross, and E. Csernok. 2010. Membrane proteinase 3 (mPR3) expression on neutrophils is not increased in localized Wegener's granulomatosis (WG) and Churg-Strauss syndrome (CSS). Clin Exp Rheumatol 28:46-50.

288. Zhang, X., Y. Dong, X. Zeng, Y. Li, and F. Tang. 2002. Clinical and pathological manifestations of patients with antineutrophil cytoplasmic autoantibodies directed against proteinase 3 or myeloperoxidase. Chin Med Sci J 17:32-35.

289. Muller, S., J. Dieker, A. Tincani, and P. L. Meroni. 2008. Pathogenic antinucleosome antibodies. Lupus 17:431-436. 
290. Fauchais, A. L., C. Martel, G. Gondran, M. Lambert, D. Launay, M. O. Jauberteau, et al. 2010. Immunological profile in primary Sjogren syndrome: clinical significance, prognosis and long-term evolution to other auto-immune disease. Autoimmunity reviews 9:595-599.

291. el-Azhary, R. A., C. C. Aponte, A. M. Nelson, A. L. Weaver, and H. A. Homburger. 2006. Antihistone antibodies in linear scleroderma variants. International journal of dermatology 45:1296-1299.

292. Frisoni, L., L. McPhie, L. Colonna, U. Sriram, M. Monestier, S. Gallucci, et al. 2005. Nuclear autoantigen translocation and autoantibody opsonization lead to increased dendritic cell phagocytosis and presentation of nuclear antigens: a novel pathogenic pathway for autoimmunity? J Immunol 175:2692-2701.

293. Radic, M., T. Marion, and M. Monestier. 2004. Nucleosomes are exposed at the cell surface in apoptosis. Journal of immunology 172:6692-6700.

294. Syed, S. H., D. Goutte-Gattat, N. Becker, S. Meyer, M. S. Shukla, J. J. Hayes, et al. 2010. Single-base resolution mapping of H1-nucleosome interactions and 3D organization of the nucleosome. Proc Natl Acad Sci U S A 107:9620-9625.

295. Phair, R. D., P. Scaffidi, C. Elbi, J. Vecerova, A. Dey, K. Ozato, et al. 2004. Global nature of dynamic protein-chromatin interactions in vivo: threedimensional genome scanning and dynamic interaction networks of chromatin proteins. Mol Cell Biol 24:6393-6402.

296. Happel, N., and D. Doenecke. 2009. Histone H1 and its isoforms: contribution to chromatin structure and function. Gene 431:1-12.

297. Wisniewski, J. R., A. Zougman, S. Kruger, and M. Mann. 2007. Mass spectrometric mapping of linker histone H1 variants reveals multiple acetylations, methylations, and phosphorylation as well as differences between cell culture and tissue. Mol Cell Proteomics 6:72-87.

298. Sarg, B., W. Helliger, H. Talasz, B. Forg, and H. H. Lindner. 2006. Histone H1 phosphorylation occurs site-specifically during interphase and mitosis: identification of a novel phosphorylation site on histone H1. J Biol Chem 281:6573-6580.

299. Zheng, Y., S. John, J. J. Pesavento, J. R. Schultz-Norton, R. L. Schiltz, S. Baek, et al. 2010. Histone $\mathrm{H} 1$ phosphorylation is associated with transcription by RNA polymerases I and II. J Cell Biol 189:407-415.

300. Cuthbert, G. L., S. Daujat, A. W. Snowden, H. Erdjument-Bromage, T. Hagiwara, M. Yamada, et al. 2004. Histone deimination antagonizes arginine methylation. Cell 118:545-553.

301. Wang, Y., J. Wysocka, J. Sayegh, Y. H. Lee, J. R. Perlin, L. Leonelli, et al. 2004. Human PAD4 regulates histone arginine methylation levels via demethylimination. Science 306:279-283.

302. Hemmers, S., J. R. Teijaro, S. Arandjelovic, and K. A. Mowen. 2011. PAD4mediated neutrophil extracellular trap formation is not required for immunity against influenza infection. PLoS One 6:e22043.

303. Dwivedi, N., J. Upadhyay, I. Neeli, S. Khan, D. Pattanaik, L. Myers, et al. 2011. Felty's syndrome autoantibodies bind to deiminated histones and neutrophil extracellular traps. Arthritis Rheum (In Press). 
304. Vitali, C., S. Bombardieri, R. Jonsson, H. M. Moutsopoulos, E. L. Alexander, S. E. Carsons, et al. 2002. Classification criteria for Sjogren's syndrome: a revised version of the European criteria proposed by the American-European Consensus Group. Ann Rheum Dis 61:554-558.

305. Subcommittee for scleroderma criteria of the American Rheumatism Association Diagnostic and Therapeutic Criteria Committee. 1980. Preliminary criteria for the classification of systemic sclerosis (scleroderma). Arthritis Rheum 23:581-590.

306. Kessenbrock, K., M. Krumbholz, U. Schonermarck, W. Back, W. L. Gross, Z. Werb, et al. 2009. Netting neutrophils in autoimmune small-vessel vasculitis. Nat Med 15:623-625.

307. Fuchs, T. A., A. Brill, D. Duerschmied, D. Schatzberg, M. Monestier, D. D. Myers, Jr., et al. 2010. Extracellular DNA traps promote thrombosis. Proc Natl Acad Sci U S A 107:15880-15885.

308. Clark, S. R., A. C. Ma, S. A. Tavener, B. McDonald, Z. Goodarzi, M. M. Kelly, et al. 2007. Platelet TLR4 activates neutrophil extracellular traps to ensnare bacteria in septic blood. Nat Med 13:463-469.

309. Berends, E. T., A. R. Horswill, N. M. Haste, M. Monestier, V. Nizet, and M. von Kockritz-Blickwede. 2010. Nuclease expression by Staphylococcus aureus facilitates escape from neutrophil extracellular traps. J Innate Immun 2:576-586.

310. von Kockritz-Blickwede, M., O. Goldmann, P. Thulin, K. Heinemann, A. NorrbyTeglund, M. Rohde, et al. 2008. Phagocytosis-independent antimicrobial activity of mast cells by means of extracellular trap formation. Blood 111:3070-3080.

311. Wang, Y., M. Li, S. Stadler, S. Correll, P. Li, D. Wang, et al. 2009. Histone hypercitrullination mediates chromatin decondensation and neutrophil extracellular trap formation. J Cell Biol 184:205-213.

312. Munks, M. W., A. S. McKee, M. K. Macleod, R. L. Powell, J. L. Degen, N. A. Reisdorph, et al. 2010. Aluminum adjuvants elicit fibrin-dependent extracellular traps in vivo. Blood 116:5191-5199.

313. Li, Y., B. Liu, E. Y. Fukudome, J. Lu, W. Chong, G. Jin, et al. 2011. Identification of citrullinated histone $\mathrm{H} 3$ as a potential serum protein biomarker in a lethal model of lipopolysaccharide-induced shock. Surgery 150:442-451.

314. Schett, G., J. Smole, C. Zimmermann, H. Hiesberger, E. Hoefler, S. Fournel, et al. 2002. The autoimmune response to chromatin antigens in systemic lupus erythematosus: autoantibodies against histone $\mathrm{H} 1$ are a highly specific marker for SLE associated with increased disease activity. Lupus 11:704-715.

315. Sun, X. Y., J. Shi, L. Han, Y. Su, and Z. G. Li. 2008. Anti-histones antibodies in systemic lupus erythematosus: prevalence and frequency in neuropsychiatric lupus. J Clin Lab Anal 22:271-277.

316. Touloupi, E., J. G. Routsias, and A. G. Tzioufas. 2005. Cross-recognition between histones and La/SSB may account for anti-DNA reactivity in SLE patients. Clin Exp Immunol 142:172-179.

317. Stummvoll, G. H., R. D. Fritsch, B. Meyer, E. Hoefler, M. Aringer, J. S. Smolen, et al. 2009. Characterisation of cellular and humoral autoimmune responses to histone $\mathrm{H} 1$ and core histones in human systemic lupus erythaematosus. Ann Rheum Dis 68:110-116.

318. Brown, D. T., T. Izard, and T. Misteli. 2006. Mapping the interaction surface of 
linker histone H1(0) with the nucleosome of native chromatin in vivo. Nat Struct Mol Biol 13:250-255.

319. George, E. M., T. Izard, S. D. Anderson, and D. T. Brown. 2010. Nucleosome interaction surface of linker histone $\mathrm{H} 1 \mathrm{c}$ is distinct from that of $\mathrm{H1}(0)$. The Journal of biological chemistry 285:20891-20896.

320. Vogler, C., C. Huber, T. Waldmann, R. Ettig, L. Braun, A. Izzo, et al. 2010. Histone $\mathrm{H} 2 \mathrm{~A}$ C-terminus regulates chromatin dynamics, remodeling, and histone H1 binding. PLoS Genet 6:e1001234.

321. Kim, K., J. Choi, K. Heo, H. Kim, D. Levens, K. Kohno, et al. 2008. Isolation and characterization of a novel H1.2 complex that acts as a repressor of p53-mediated transcription. $J$ Biol Chem 283:9113-9126.

322. Mackey-Cushman, S. L., J. Gao, D. A. Holmes, J. I. Nunoya, R. Wang, D. Unutmaz, et al. 2011. FoxP3 interacts with linker histone H1.5 to modulate gene expression and program Treg cell activity. Genes Immun 12:559-567.

323. Medzhitov, R., and C. A. Janeway, Jr. 2002. Decoding the patterns of self and nonself by the innate immune system. Science 296:298-300.

324. Urban, C. F., D. Ermert, M. Schmid, U. Abu-Abed, C. Goosmann, W. Nacken, et al. 2009. Neutrophil extracellular traps contain calprotectin, a cytosolic protein complex involved in host defense against Candida albicans. PLoS Pathog 5:e1000639.

325. Senshu, T., K. Akiyama, and K. Nomura. 1999. Identification of citrulline residues in the $\mathrm{V}$ subdomains of keratin $\mathrm{K} 1$ derived from the cornified layer of newborn mouse epidermis. Exp Dermatol 8:392-401.

326. Sebbag, M., S. Chapuy-Regaud, I. Auger, E. Petit-Texeira, C. Clavel, L. Nogueira, et al. 2004. Clinical and pathophysiological significance of the autoimmune response to citrullinated proteins in rheumatoid arthritis. Joint Bone Spine 71:493-502.

327. Hagiwara, T., K. Nakashima, H. Hirano, T. Senshu, and M. Yamada. 2002. Deimination of arginine residues in nucleophosmin/B23 and histones in HL-60 granulocytes. Biochem Biophys Res Commun 290:979-983.

328. Garcia-Romo, G. S., S. Caielli, B. Vega, J. Connolly, F. Allantaz, Z. Xu, et al. 2011. Netting neutrophils are major inducers of type I IFN production in pediatric systemic lupus erythematosus. Sci Transl Med 3:73ra20.

329. Lande, R., D. Ganguly, V. Facchinetti, L. Frasca, C. Conrad, J. Gregorio, et al. 2011. Neutrophils activate plasmacytoid dendritic cells by releasing self-DNApeptide complexes in systemic lupus erythematosus. Sci Transl Med 3:73ra19.

330. Manolova, I., M. Dancheva, and K. Halacheva. 2001. Antineutrophil cytoplasmic antibodies in patients with systemic lupus erythematosus: prevalence, antigen specificity, and clinical associations. Rheumatol Int 20:197-204.

331. Tamiya, H., K. Tani, J. Miyata, K. Sato, T. Urata, B. Lkhagvaa, et al. 2006. Defensins- and cathepsin G-ANCA in systemic lupus erythematosus. Rheumatol Int 27:147-152.

332. Sugawara, K., Y. Yoshizawa, S. Tzeng, W. L. Epstein, and K. Fukuyama. 1998. Colorimetric determination of citrulline residues in proteins. Anal Biochem 265:92-96.

333. Moens, U., O. M. Seternes, A. W. Hey, Y. Silsand, T. Traavik, B. Johansen, et al. 
1995. In vivo expression of a single viral DNA-binding protein generates systemic lupus erythematosus-related autoimmunity to double-stranded DNA and histones. Proc Natl Acad Sci U S A 92:12393-12397.

334. Kalaaji, M., G. Sturfelt, J. E. Mjelle, H. Nossent, and O. P. Rekvig. 2006. Critical comparative analyses of anti-alpha-actinin and glomerulus-bound antibodies in human and murine lupus nephritis. Arthritis Rheum 54:914-926.

335. Andreassen, K., G. Bredholt, U. Moens, S. Bendiksen, G. Kauric, and O. P. Rekvig. 1999. T cell lines specific for polyomavirus T-antigen recognize Tantigen complexed with nucleosomes: a molecular basis for anti-DNA antibody production. Eur J Immunol 29:2715-2728.

336. Bredholt, G., O. P. Rekvig, K. Andreassen, U. Moens, and T. N. Marion. 2001. Differences in the reactivity of CD4+ T-cell lines generated against free versus nucleosome-bound SV40 large T antigen. Scand J Immunol 53:372-380.

337. Monestier, M., T. M. Fasy, M. J. Losman, K. E. Novick, and S. Muller. 1993. Structure and binding properties of monoclonal antibodies to core histones from autoimmune mice. Mol Immunol 30:1069-1075.

338. Kesteman, N., G. Vansanten, B. Pajak, S. M. Goyert, and M. Moser. 2008. Injection of lipopolysaccharide induces the migration of splenic neutrophils to the $\mathrm{T}$ cell area of the white pulp: role of CD14 and CXC chemokines. J Leukoc Biol 83:640-647.

339. Pulendran, B., G. Kannourakis, S. Nouri, K. G. Smith, and G. J. Nossal. 1995. Soluble antigen can cause enhanced apoptosis of germinal-centre B cells. Nature 375:331-334

340. Shokat, K. M., and C. C. Goodnow. 1995. Antigen-induced B-cell death and elimination during germinal-centre immune responses. Nature 375:334-338.

341. Radic, M., M. Herrmann, J. van der Vlag, and O. P. Rekvig. 2011. Regulatory and pathogenetic mechanisms of autoantibodies in SLE. Autoimmunity 44:349-356.

342. Shlomchik, M. J., A. Marshak-Rothstein, C. B. Wolfowicz, T. L. Rothstein, and M. G. Weigert. 1987. The role of clonal selection and somatic mutation in autoimmunity. Nature 328:805-811.

343. Shlomchik, M. J., A. H. Aucoin, D. S. Pisetsky, and M. G. Weigert. 1987. Structure and function of anti-DNA autoantibodies derived from a single autoimmune mouse. Proc Natl Acad Sci U S A 84:9150-9154.

344. Shlomchik, M., M. Mascelli, H. Shan, M. Z. Radic, D. Pisetsky, A. MarshakRothstein, et al. 1990. Anti-DNA antibodies from autoimmune mice arise by clonal expansion and somatic mutation. $J$ Exp Med 171:265-292.

345. Radic, M. Z., and M. Weigert. 1994. Genetic and structural evidence for antigen selection of anti-DNA antibodies. Anпи Rev Immunol 12:487-520.

346. van Bavel, C. C., J. W. Dieker, Y. Kroeze, W. P. Tamboer, R. Voll, S. Muller, et al. 2011. Apoptosis-induced histone $\mathrm{H} 3$ methylation is targeted by autoantibodies in systemic lupus erythematosus. Ann Rheum Dis 70:201-207.

347. Schachna, L., F. M. Wigley, S. Morris, A. C. Gelber, A. Rosen, and L. CasciolaRosen. 2002. Recognition of Granzyme B-generated autoantigen fragments in scleroderma patients with ischemic digital loss. Arthritis Rheum 46:1873-1884. 
348. Ohmori, H., and N. Kanayama. 2005. Immunogenicity of an inflammationassociated product, tyrosine nitrated self-proteins. Autoimmun Rev 4:224-229.

349. Asaga, H., K. Nakashima, T. Senshu, A. Ishigami, and M. Yamada. 2001. Immunocytochemical localization of peptidylarginine deiminase in human eosinophils and neutrophils. $J$ Leukoc Biol 70:46-51.

350. Vossenaar, E. R., T. R. Radstake, A. van der Heijden, M. A. van Mansum, C. Dieteren, D. J. de Rooij, et al. 2004. Expression and activity of citrullinating peptidylarginine deiminase enzymes in monocytes and macrophages. Ann Rheum Dis 63:373-381.

351. Arita, K., T. Shimizu, H. Hashimoto, Y. Hidaka, M. Yamada, and M. Sato. 2006. Structural basis for histone $\mathrm{N}$-terminal recognition by human peptidylarginine deiminase 4. Proc Natl Acad Sci U S A 103:5291-5296.

352. Lum, J. J., G. Bren, R. McClure, and A. D. Badley. 2005. Elimination of senescent neutrophils by TNF-related apoptosis-inducing [corrected] ligand. $J$ Immunol 175:1232-1238.

353. Furze, R. C., and S. M. Rankin. 2008. The role of the bone marrow in neutrophil clearance under homeostatic conditions in the mouse. FASEB J 22:3111-3119.

354. Ramos-Kichik, V., R. Mondragon-Flores, M. Mondragon-Castelan, S. GonzalezPozos, S. Muniz-Hernandez, O. Rojas-Espinosa, et al. 2009. Neutrophil extracellular traps are induced by Mycobacterium tuberculosis. Tuberculosis (Edinb) 89:29-37.

355. Bruns, S., O. Kniemeyer, M. Hasenberg, V. Aimanianda, S. Nietzsche, A. Thywissen, et al. 2010. Production of extracellular traps against Aspergillus fumigatus in vitro and in infected lung tissue is dependent on invading neutrophils and influenced by hydrophobin RodA. PLoS Pathog 6:e1000873.

356. McCormick, A., L. Heesemann, J. Wagener, V. Marcos, D. Hartl, J. Loeffler, et al. 2010. NETs formed by human neutrophils inhibit growth of the pathogenic mold Aspergillus fumigatus. Microbes Infect 12:928-936.

357. Ma, A. C., and P. Kubes. 2008. Platelets, neutrophils, and neutrophil extracellular traps (NETs) in sepsis. $J$ Thromb Haemost 6:415-420.

358. Margraf, S., T. Logters, J. Reipen, J. Altrichter, M. Scholz, and J. Windolf. 2008. Neutrophil-derived circulating free DNA (cf-DNA/NETs): a potential prognostic marker for posttraumatic development of inflammatory second hit and sepsis. Shock 30:352-358.

359. Palatinus, A., and M. Adams. 2009. Thrombosis in systemic lupus erythematosus. Semin Thromb Hemost 35:621-629.

360. Rosenstein, E. D., and N. Kramer. 1991. Felty's and pseudo-Felty's syndromes. Semin Arthritis Rheum 21:129-142.

361. Campion, G., P. J. Maddison, N. Goulding, I. James, M. J. Ahern, I. Watt, et al. 1990. The Felty syndrome: a case-matched study of clinical manifestations and outcome, serologic features, and immunogenetic associations. Medicine (Baltimore) 69:69-80.

362. Breedveld, F. C., G. J. Lafeber, E. de Vries, J. H. van Krieken, and A. Cats. 1986. Immune complexes and the pathogenesis of neutropenia in Felty's syndrome. Ann Rheum Dis 45:696-702. 
363. Starkebaum, G., W. P. Arend, F. A. Nardella, and S. E. Gavin. 1980.

Characterization of immune complexes and immunoglobulin $\mathrm{G}$ antibodies reactive with neutrophils in the sera of patients with Felty's syndrome. J Lab Clin Med 96:238-251.

364. van Krieken, J. H., F. C. Breedveld, and J. te Velde. 1988. The spleen in Felty's syndrome: a histological, morphometrical, and immunohistochemical study. Eur $J$ Haematol 40:58-64.

365. Turesson, C., L. T. Jacobsson, G. Sturfelt, E. L. Matteson, L. Mathsson, and J. Ronnelid. 2007. Rheumatoid factor and antibodies to cyclic citrullinated peptides are associated with severe extra-articular manifestations in rheumatoid arthritis. Ann Rheum Dis 66:59-64.

366. Alexiou, I., A. Germenis, A. Koutroumpas, A. Kontogianni, K. Theodoridou, and L. I. Sakkas. 2008. Anti-cyclic citrullinated peptide-2 (CCP2) autoantibodies and extra-articular manifestations in Greek patients with rheumatoid arthritis. Clin Rheumatol 27:511-513.

367. van Venrooij, W. J., E. R. Vossenaar, and A. J. Zendman. 2004. Anti-CCP antibodies: the new rheumatoid factor in the serology of rheumatoid arthritis. Autoimmun Rev 3 Suppl 1:S17-19.

368. Thacker, S. G., D. Duquaine, J. Park, and M. J. Kaplan. 2010. Lupus-prone New Zealand Black/New Zealand White F1 mice display endothelial dysfunction and abnormal phenotype and function of endothelial progenitor cells. Lupus 19:288299.

369. Perry, D., A. Sang, Y. Yin, Y. Y. Zheng, and L. Morel. 2011. Murine models of systemic lupus erythematosus. J Biomed Biotechnol 2011:271694.

370. Tonegawa, S. 1983. Somatic generation of antibody diversity. Nature 302:575581.

371. Basten, A., and P. A. Silveira. 2010. B-cell tolerance: mechanisms and implications. Curr Opin Immunol 22:566-574.

372. Gay, D., T. Saunders, S. Camper, and M. Weigert. 1993. Receptor editing: an approach by autoreactive B cells to escape tolerance. J Exp Med 177:999-1008.

373. Tiegs, S. L., D. M. Russell, and D. Nemazee. 1993. Receptor editing in selfreactive bone marrow B cells. J Exp Med 177:1009-1020.

374. Khan, S. N., E. J. Witsch, N. G. Goodman, A. K. Panigrahi, C. Chen, Y. Jiang, et al. 2008. Editing and escape from editing in anti-DNA B cells. Proc Natl Acad Sci US A 105:3861-3866.

375. Clark, M. R., A. Tanaka, S. E. Powers, and M. Veselits. 2011. Receptors, subcellular compartments and the regulation of peripheral B cell responses: the illuminating state of anergy. Mol Immunol 48:1281-1286.

376. Hill, J. A., D. A. Bell, W. Brintnell, D. Yue, B. Wehrli, A. M. Jevnikar, et al. 2008. Arthritis induced by posttranslationally modified (citrullinated) fibrinogen in DR4-IE transgenic mice. J Exp Med 205:967-979.

377. Hida, S., N. N. Miura, Y. Adachi, and N. Ohno. 2004. Influence of arginine deimination on antigenicity of fibrinogen. $J$ Autoimmun 23:141-150.

378. Thoma, F., T. Koller, and A. Klug. 1979. Involvement of histone H1 in the organization of the nucleosome and of the salt-dependent superstructures of chromatin. J Cell Biol 83:403-427. 
379. Ramakrishnan, V. 1997. Histone H1 and chromatin higher-order structure. Crit Rev Eukaryot Gene Expr 7:215-230.

380. Bednar, J., R. A. Horowitz, S. A. Grigoryev, L. M. Carruthers, J. C. Hansen, A. J. Koster, et al. 1998. Nucleosomes, linker DNA, and linker histone form a unique structural motif that directs the higher-order folding and compaction of chromatin. Proc Natl Acad Sci U S A 95:14173-14178.

381. Allan, J., P. G. Hartman, C. Crane-Robinson, and F. X. Aviles. 1980. The structure of histone $\mathrm{H} 1$ and its location in chromatin. Nature 288:675-679.

382. Roque, A., I. Ponte, J. L. Arrondo, and P. Suau. 2008. Phosphorylation of the carboxy-terminal domain of histone H1: effects on secondary structure and DNA condensation. Nucleic Acids Res 36:4719-4726.

383. Thomas, J. O. 1999. Histone H1: location and role. Curr Opin Cell Biol 11:312317.

384. Noll, M., and R. D. Kornberg. 1977. Action of micrococcal nuclease on chromatin and the location of histone H1. J Mol Biol 109:393-404.

385. Brown, D. T., A. Gunjan, B. T. Alexander, and D. B. Sittman. 1997. Differential effect of $\mathrm{H} 1$ variant overproduction on gene expression is due to differences in the central globular domain. Nucleic Acids Res 25:5003-5009.

386. Vermaak, D., O. C. Steinbach, S. Dimitrov, R. A. Rupp, and A. P. Wolffe. 1998. The globular domain of histone $\mathrm{H} 1$ is sufficient to direct specific gene repression in early Xenopus embryos. Curr Biol 8:533-536.

387. Cirillo, L. A., C. E. McPherson, P. Bossard, K. Stevens, S. Cherian, E. Y. Shim, et al. 1998. Binding of the winged-helix transcription factor HNF3 to a linker histone site on the nucleosome. EMBOJ 17:244-254.

388. Engelmann, R., M. Eggert, G. Neeck, and B. Mueller-Hilke. 2010. The impact of HLA-DRB alleles on the subclass titres of antibodies against citrullinated peptides. Rheumatology (Oxford) 49:1862-1866.

389. Loughran, T. P., Jr., and G. Starkebaum. 1987. Large granular lymphocyte leukemia. Report of 38 cases and review of the literature. Medicine (Baltimore) 66:397-405.

390. Prochorec-Sobieszek, M., G. Rymkiewicz, H. Makuch-Lasica, M. Majewski, K. Michalak, R. Rupinski, et al. 2008. Characteristics of T-cell large granular lymphocyte proliferations associated with neutropenia and inflammatory arthropathy. Arthritis Res Ther 10:R55.

391. Starkebaum, G., T. P. Loughran, Jr., L. K. Gaur, P. Davis, and B. S. Nepom. 1997. Immunogenetic similarities between patients with Felty's syndrome and those with clonal expansions of large granular lymphocytes in rheumatoid arthritis. Arthritis Rheum 40:624-626.

392. Langerak, A. W., Y. Sandberg, and J. J. van Dongen. 2003. Spectrum of T-large granular lymphocyte lymphoproliferations: ranging from expanded activated effector T cells to T-cell leukaemia. Br J Haematol 123:561-562.

393. Osuji, N., K. Beiske, U. Randen, E. Matutes, G. Tjonnfjord, D. Catovsky, et al. 2007. Characteristic appearances of the bone marrow in T-cell large granular lymphocyte leukaemia. Histopathology 50:547-554.

394. Burks, E. J., and T. P. Loughran, Jr. 2006. Pathogenesis of neutropenia in large granular lymphocyte leukemia and Felty syndrome. Blood Rev 20:245-266. 
395. Starkebaum, G., J. W. Singer, and W. P. Arend. 1980. Humoral and cellular immune mechanisms of neutropenia in patients with Felty's syndrome. Clin Exp Immunol 39:307-314.

396. Woodfin, A., M. B. Voisin, and S. Nourshargh. 2010. Recent developments and complexities in neutrophil transmigration. Curr Opin Hematol 17:9-17.

397. Kitsis, E., and G. Weissmann. 1991. The role of the neutrophil in rheumatoid arthritis. Clin Orthop Relat Res:63-72. 


\section{VITA}

Nishant Dwivedi was born in Bhopal, India in the year 1981. He graduated from high school in 1998. At school, he was the recipient of State Science Talent Search and National Talent Search Examination (NTSE) scholarships given by State Government of Madhya Pradesh and Government of India respectively. He obtained his Bachelor of Medicine, Bachelor of Surgery (M.B., B.S.) degree from Lokmanya Tilak Municipal Medical College (LTMMC), Mumbai affiliated to Maharashtra University of Health Sciences (MUHS), Nashik in April 2004. He joined the Integrated Program in Biomedical Sciences (IPBS) at the University of Tennessee Health Science Center (UTHSC), Memphis in August 2005 to work for his Ph.D. 